\title{
Spatial-temporal actin dynamics during synaptic plasticity of single dendritic spine investigated by two- photon fluorescence correlation spectroscopy
}

\author{
Dissertation
}

for the award of the degree

"Doctor rerum naturalium"

of the Georg-August-Universität Göttingen

within the doctoral program (IMPRS-Physics of biological and complex systems)

of the Georg-August University School of Science (GAUSS)

\author{
submitted by \\ Jian Hua Chen \\ from Nantou,Taiwan
}

Göttingen 2013 
Members of the Thesis Committee:

Prof. Dr. Peter Jomo Walla (Referee)

Group of Biomolecular Spectroscopy and Single-Molecule Detection

Max Planck Institute for Biophysical Chemistry, Göttingen

and

Department of Biophysical Chemistry

Institute for Physical and Theoretical Chemistry

Technische Universität Braunschweig

Prof. Dr. Reinhard Jahn

Department of Neurobiology

Max Planck Institute for Biophysical Chemistry, Göttingen

Prof. Dr. Andreas Janshoff

Institute for Physical Chemistry

Georg-August-University Göttingen 


\section{Declaration}

I hereby declare that my $\mathrm{Ph} . \mathrm{D}$. thesis 'Spatial-temporal actin dynamics during synaptic plasticity of single dendritic spine investigated by two photon fluorescence correlation spectroscopy' has been written independently with no other aids or sources than quoted.

Göttingen, June 5th 2013

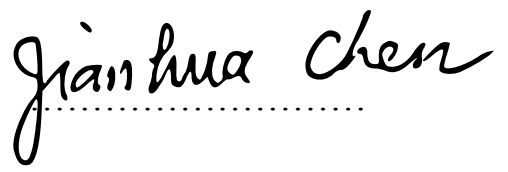




\section{Contents}

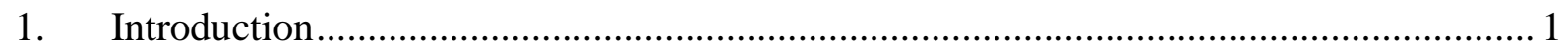

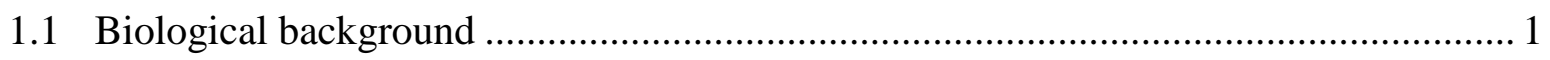

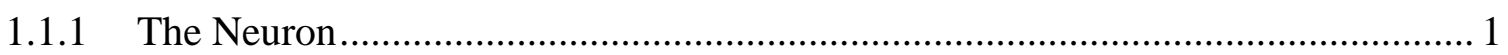

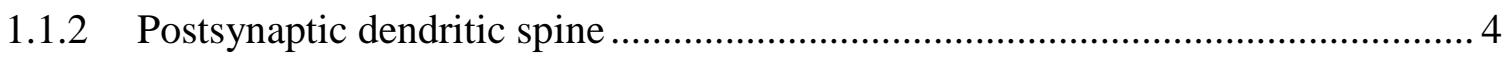

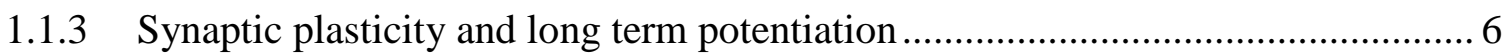

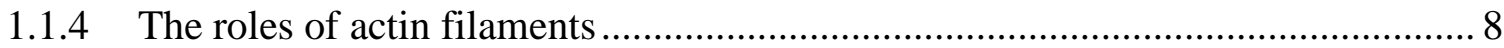

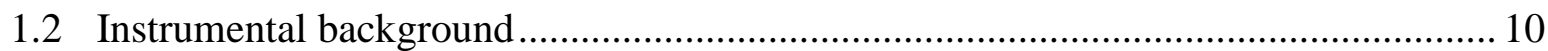

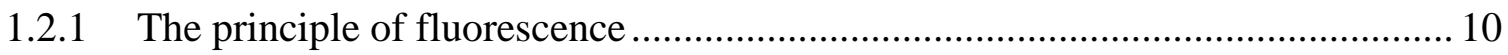

1.2.2 Fluorescence correlation spectroscopy (FCS) ................................................ 16

1.2.3 Focal volume (OPE and TPE) and diffusion coefficient................................... 21

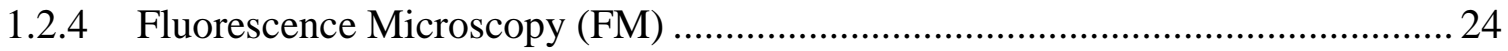

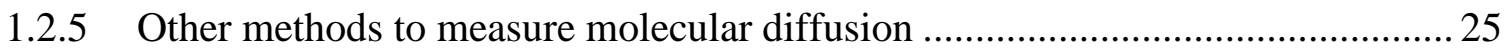

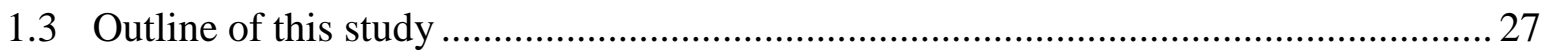

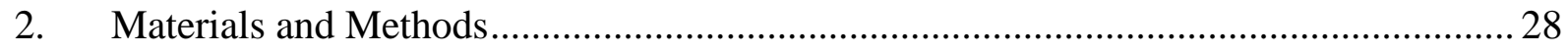

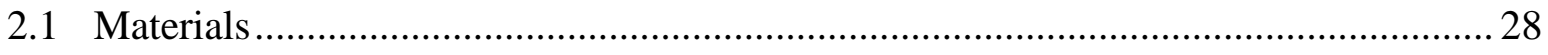

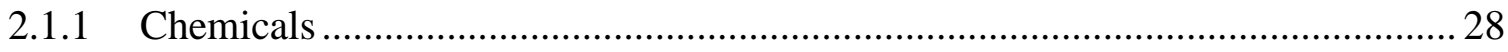

2.1.2 Preparation of organotypic hippocampus slices .......................................... 28

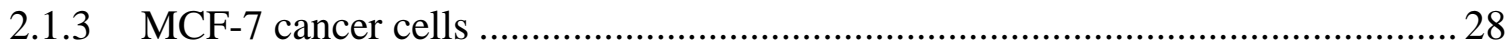

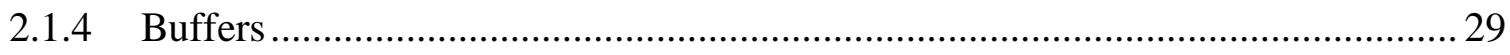


2.1.5 Living cell chamber system.

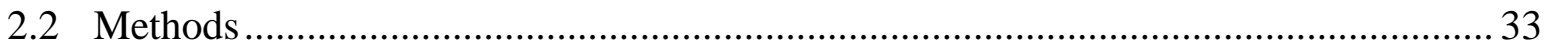

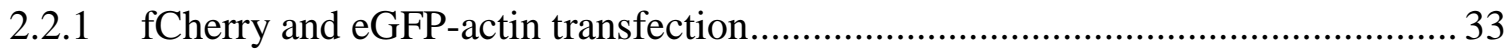

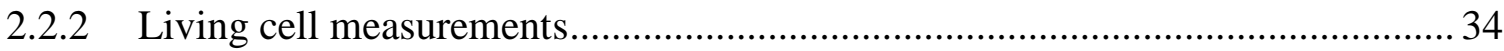

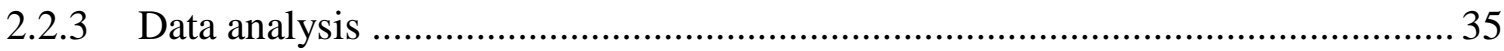

2.2.4 Two photon fluorescence correlation spectroscopy (2P-FCS) and Wide-field

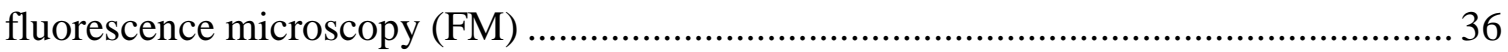

2.2.5 Co-localization of focal plane between 2P-FCS and FM.................................. 38

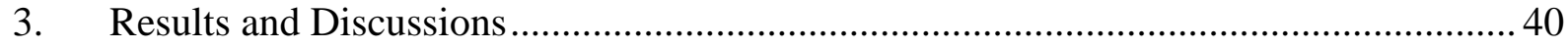

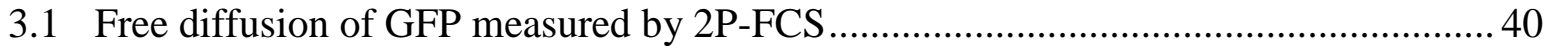

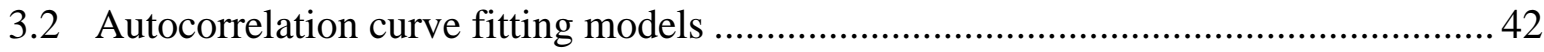

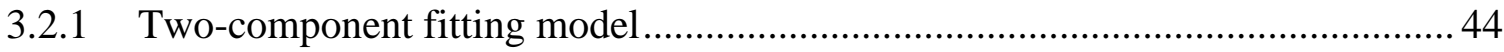

3.2.2 Multiple diffusion species with different brightness ..................................... 47

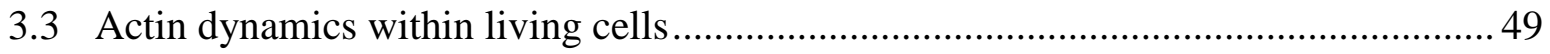

3.3.1 The treatment of Lat-A in MCF-7 cancer cells .................................................. 50

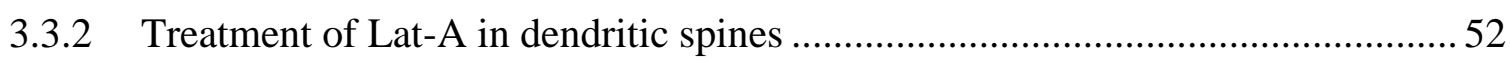

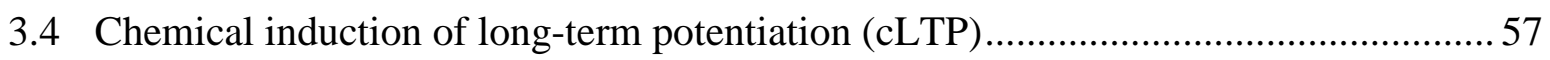

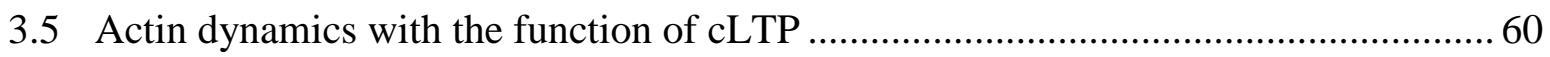

3.5.1 Actin dynamics within dendritic spine studied by FRAP .................................. 61

3.5.2 Actin dynamics within dendritic spine studied by 2P-FCS.............................63

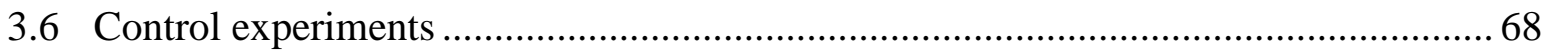

3.6.1 Actin dynamics under cLTP with the treatment of AP5 and Verapamil .............68 
3.6.2 TEA stimulation without morphological changes

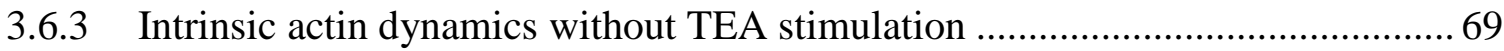

3.7 Summary of molecular dynamics within living cells .......................................... 75

3.8 Remodeling and reorganization of actin filaments during synaptic plasticity ............ 77

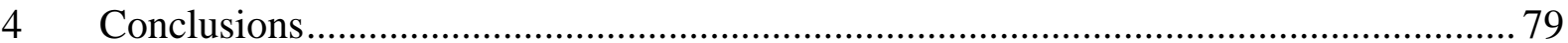

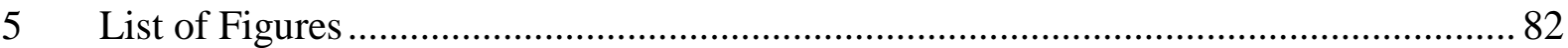

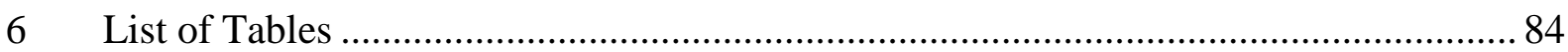

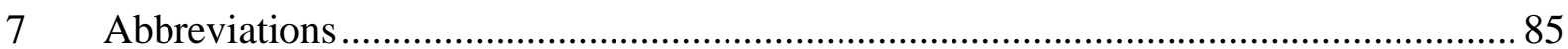

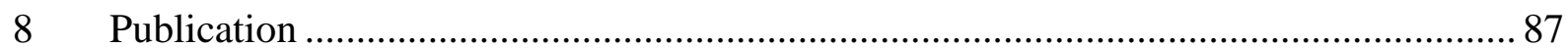

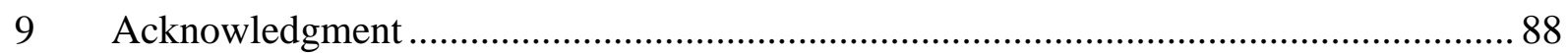

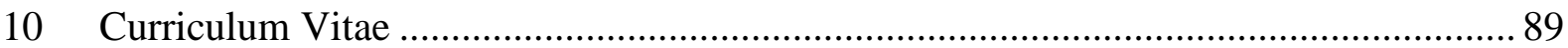

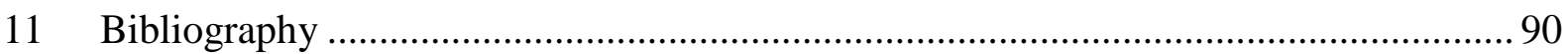




\section{Introduction}

\subsection{Biological background}

\subsubsection{The Neuron}

The way people think, act and remember is related to the complicated functions of the brain. Our brains perceive information, process it and then store it as short-term and long-term memories and even generate behavior harmonically. There are at least 100 billion nerve cells (neurons) that control and coordinate the communication within the human brain. They are classified by their diverse morphologies and functionalities. However, they share some integral parts. The basic compositions of a neuron are cell body (soma), axon, dendrite and synapse (Levitan \& Kaczmarek, 2002; Spruston, 2008). As illustrated in Figure 1.1.1, basically, there are two different domains, i.e. the basal and apical domain that stretch out in opposite directions from the cell body. On the basal side the dendrite extends out of the cell body and typically has multiple branches in the terminals (Harvey et al, 2000).

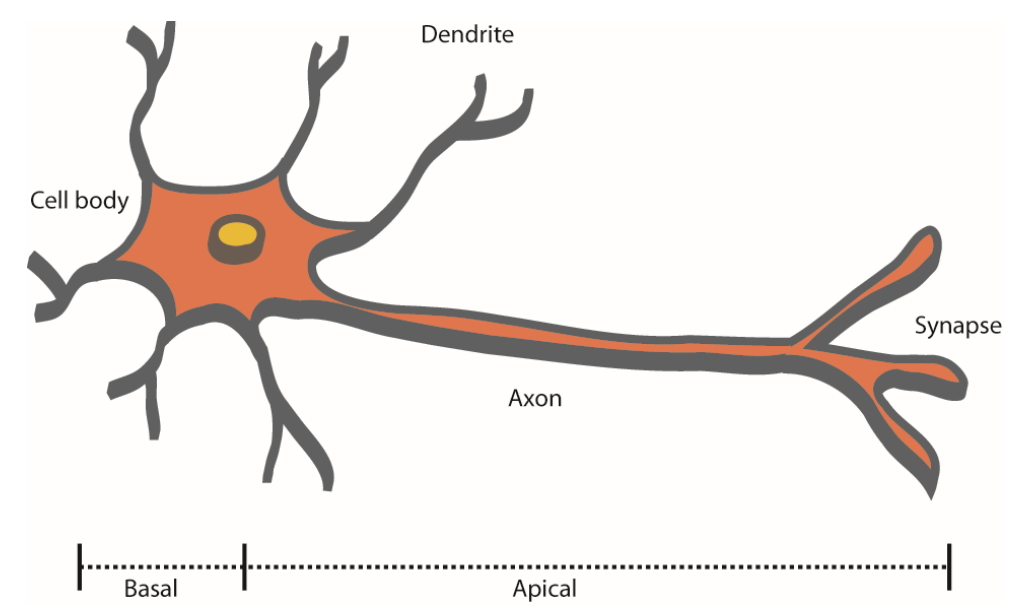

Figure 1.1.1 The basic structure of a single neuron. The major parts of the neuron cell are cell body (soma), dendrite, axon and synapse where nerve cell transfers electrical information to chemical signals 
The axon stretches out from the cell body on the apical side and extends with a constant diameter over a long distance. Axons are specialized for the delivery of action potentials, which transfer electrical signal rapidly. Typically, the information input from other cells is received by dendrites while the output of the signals is sent through the axon terminals. At the axonal terminal, the electrical signal is transformed into a chemical signal by triggering the release of synaptic vesicles. These synaptic vesicles contain neurotransmitters, which in turn diffuse across the synaptic cleft, and bind to the post-synaptic receptors located on the dendritic terminal. The receptor is usually a ligand-gated ion channel, so the binding of neurotransmitters opens the ion channel and thus depolarizes or hyperpolarizes the postsynaptic neuron. Here, the chemical signal is again transformed into electrical one, and the signal is transmitted to the next cell.

Most of the relevant proteins and membranes are synthesized in the cell body of a neuron. They are packed later into vesicles and transported to the cell terminals along special cytoskeleton components like actin filaments or microtubules. Upon the arrival of the vesicles to the axon terminal synapse (presynaptic cell), several complicated mechanisms lead to vesicle docking and fusion with the plasma membrane and the subsequent release of neurotransmitter, which is mainly composed of glutamate (Cypionka et al, 2009; Hernandez et al, 2012; Vennekate et al, 2012; Zwilling et al, 2007). The receiving part is called a postsynaptic cell and will be explained in detail in the next section. 
(A)

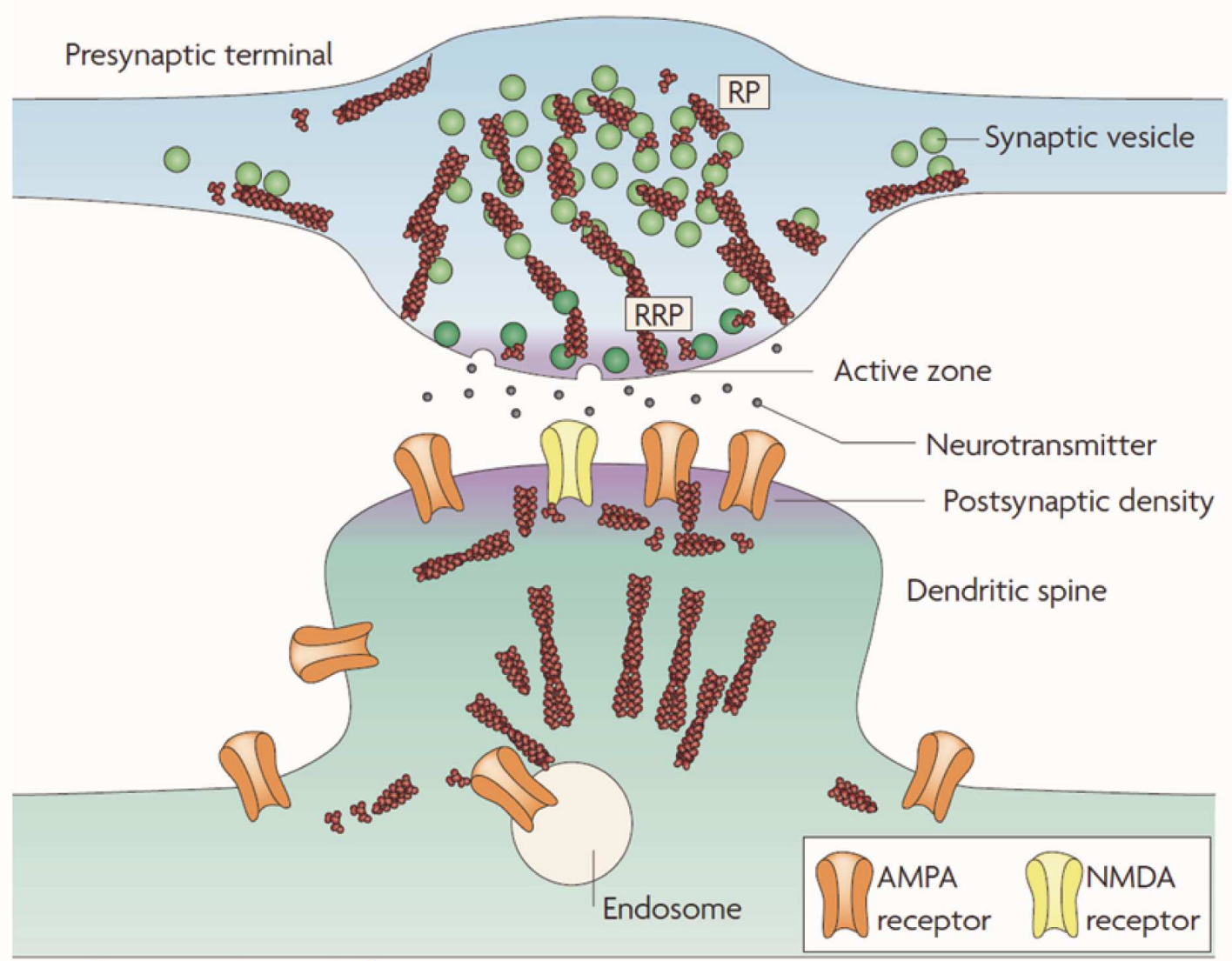

Postsynaptic terminal

(B)

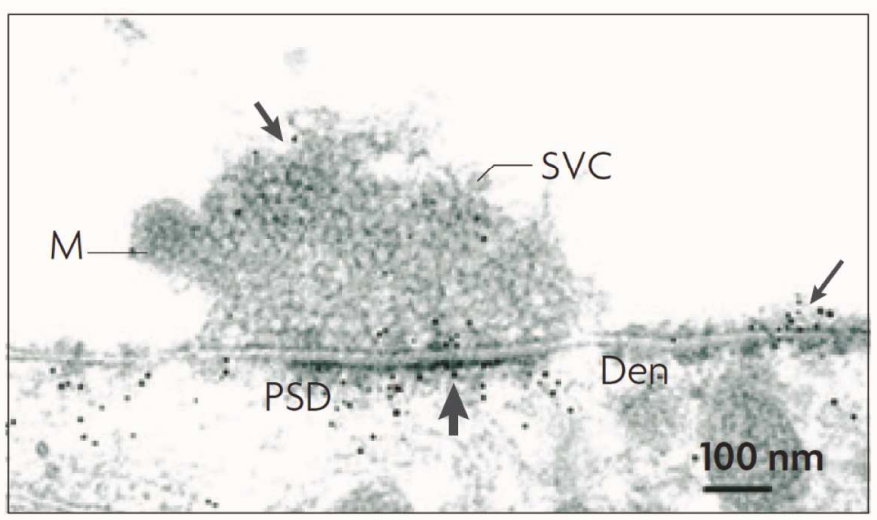

(C)

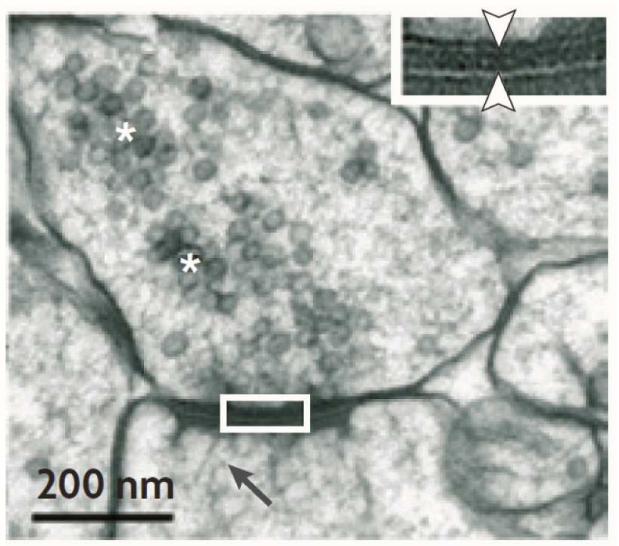

Figure 1.1.2 Overview of the pre- and post-synaptic cells. (A) Synaptic vesicles are transported to the axon terminal and neurotransmitters are released. Chemical stimulation can induce functional and morphological changes in the postsynaptic sites. (B) and (C) Electron micrographs of synapse, actin was highlighted by arrows or asterisks. [Figure modified with permission from (Cingolani \& Goda, 2008)] 


\subsubsection{Postsynaptic dendritic spine}

Dendritic spines are tiny protrusions from the dendritic sites of the basal domain, which exhibit high heterogeneities in size and morphology, as demonstrated in Figure 1.1.3, and are often shorter than axons. The diameter of dendritic spines is in the sub-micron range (Kasai et al, 2010). In the brain, dendritic spines are the major sites to receive synaptic excitatory input in the cortex and have been largely studied because the structural change is closely related to processes of learning and memory (Lamprecht \& LeDoux, 2004; Yuste \& Bonhoeffer, 2001). The functions of the neuronal network are controlled and regulated precisely by neuronal activities and the heterogeneity of spine size and shape leads to the functional diversities. Neuronal diseases such as Parkinson's and Alzheimer diseases strike, if the intricate network malfunctions (Lin \& Koleske, 2010).

As shown in Figure 1.1.2 (A), there are certain protein receptors on the plasma membrane of dendritic spine. The interaction of released neurotransmitters and the postsynaptic receptors, such as $N$-methyl-D-aspartate receptors (NMDAR) or $\alpha$-amino-3-hydroxy5-methyl-4-isoxazolepropionic acid receptors (AMPAR), result in down-stream signal transductions that may later trigger functional or morphological changes of dendritic spines (Harvey et al, 2008; Lee et al, 2009; Yasuda et al, 2006). Often two important parts of a single dendritic spine can be distinguished, (c.f. Figure 1.1.3), namely the spine head and the spine neck that connects the spine head to the dendritic shaft. The spine neck hinders the influx and outflux of the diffusion molecules, and makes the dendritic spine an isolated microenvironment (Harvey et al, 2008; Kennedy et al, 2005; Yasuda et al, 2006). In short, the postsynaptic unit is a vital place where the signal transduction that may lead to long-term synaptic plasticity occurs. And such synaptic plasticity is highly related to the learning and memory processes.

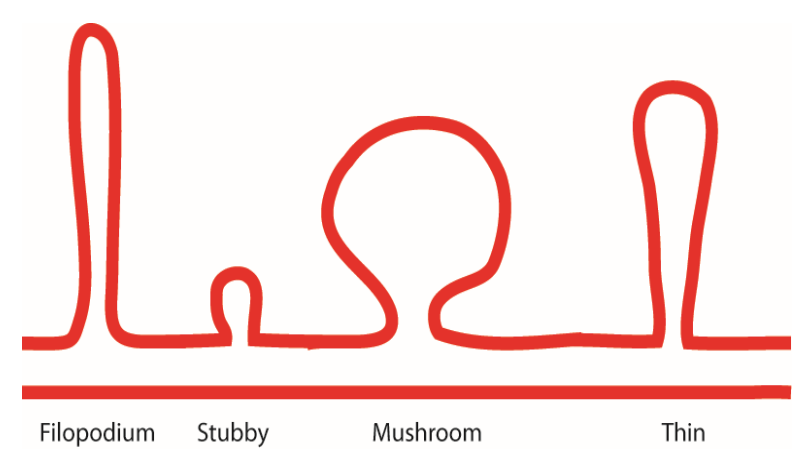

of neck) and thin (lack of head) spines can also be found in nerve cells.
Figure 1.1.3 Morphology and size heterogeneity of dendritic spines. The typical dendritic spine consists of a spine head protrusion, which is connected to the dendritic shaft by a narrow neck (mushroom type). Filopodium type dendritic spines are regarded as the spine precursor. Stubby (lack 
There is a narrow gap $(\sim 20 \mathrm{~nm})$ called synaptic cleft between the presynaptic cell and the postsynaptic cell. The existence of this gap, illustrated in Figure 1.1.2(C), implies that there is no direct connection between those two parts. The gap may provide a buffer matrix for some important trans-synaptic molecule complexes, such as cell adhesion molecules (Schmid \& Hollmann, 2010). On the tips of the postsynaptic cell, protein receptors as well as their related signal protein complexes, postsynaptic density (PSD), are concentrated, as shown in Figure 1.1.2(B). This area is unique for chemical synapse and directly faces the active zone of the presynaptic cell that is separated by the synaptic cleft (Sheng \& Hoogenraad, 2007). Furthermore, the dimensions of the spine head are highly dependent on the size of the active zone and PSD (Kasai et al, 2003). 


\subsubsection{Synaptic plasticity and long term potentiation}

The changes in synaptic transmission and connectivity allow neuron networks to store information during the process of learning and memory. As mentioned earlier in section 1.1.2, dendritic spines are highly heterogeneous in their morphological structures. Furthermore, dendritic spines are also highly dynamic in the form of elimination or generation. Such dynamic changes of postsynaptic dendritic spines are thought to contribute to the plasticity of neuronal network in the brain, and thus are correlated with the learning and memory processes (Lamprecht \& LeDoux, 2004; Yuste \& Bonhoeffer, 2001).

The changes in spine morphology are related to the functional differences of synapses. Long-term potentiation (LTP) and long-term depression (LTD) are two major parts of long lasting synaptic plasticity, which will exhibit morphological enlargements and shrinkages, respectively (Matsuzaki et al, 2004; Okamoto et al, 2004; Zhou et al, 2004). Furthermore, during the processes of LTP or LTD, not only does the spine morphology change but also the receptor trafficking on the plasma membrane is involved. For example, the trafficking of AMPAR contributes to LTP (Gu et al, 2010; Harvey et al, 2008; Lee et al, 2009; Lisman et al, 2012). During LTP, the expression number of AMPAR in the postsynaptic spine head increased by means of postsynaptic exocytosis and is correlated with actin filaments and PSD proteins (Malinow \& Malenka, 2002). It has also been suggested that the size of PSD is related to the size of spine head during these long lasting processes (Harris et al, 1992).

Besides AMPAR, NMDAR also play an important role during synaptic plasticity (Collingridge \& Bliss, 1987; Matsuzaki et al, 2004). NMDAR is a voltage-gated ion channel that is crucial for synaptogenesis and activity-dependent long lasting synaptic remodeling. Specifically, NMDAR is a $\mathrm{Ca}^{2+}$ permeable ion channel receptor, which is regarded as a coincidence detector between presynaptic and postsynaptic firing (Bliss \& Collingridge, 1993; Collingridge \& Bliss, 1987; Morris et al, 1986). It is located on the plasma membrane of PSD region at the postsynaptic site and is structurally linked to several important signaling protein complexes, such as PSD protein of $95 \mathrm{kDa}$ (PSD-95) (Kim \& Sheng, 2004). PSD-95 anchors NMDAR in the PSD area and is vital to intracellular trafficking or NMDAR transportation.

The mechanism of LTP induction involves NMDAR serving as the trigger of the LTP process that opens the voltage-gated ion channel in the early stimulation stage (Bliss \& Collingridge, 1993). During the continuing depolarization of the postsynaptic membrane and followed by the release of $\mathrm{Mg}^{2+}$ on NMDAR, the intracellular concentration of $\mathrm{Ca}^{2+}$ is 
elevated due to the NMDAR $\mathrm{Ca}^{2+}$ permeability. Due to the elevation of $\mathrm{Ca}^{2+}$ concentration, the dendritic spine undergoes further activations by other signal transduction pathways. One of the important events is the insertion of AMPAR into the plasma membrane. Other accompanying events including morphological changes of dendritic spines and protein trafficking are thought to be tightly related to the modulations of cytoskeleton structure, especially to actin filaments (Fischer et al, 1998; Murakoshi et al, 2011; Star et al, 2002).

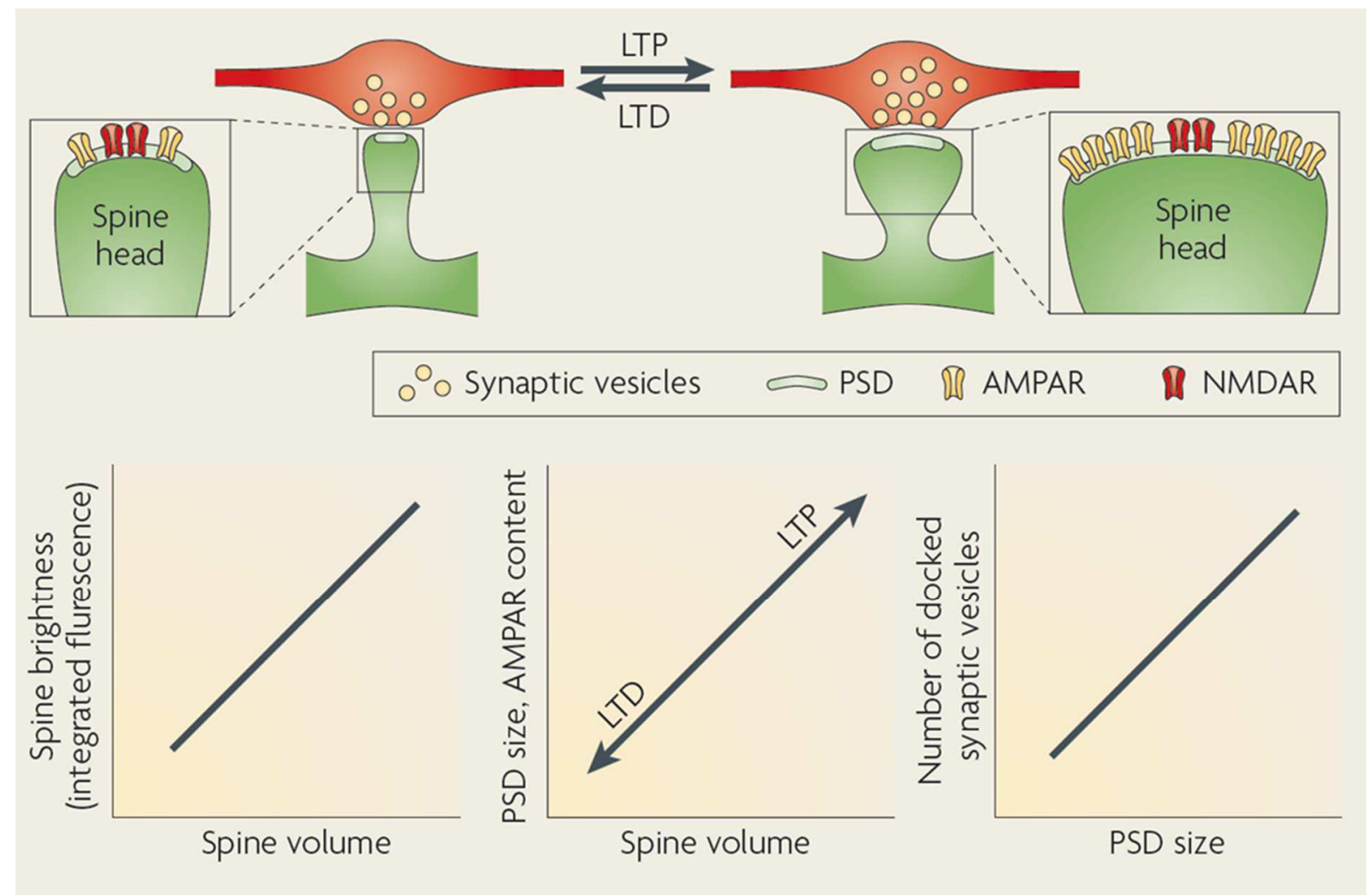

Figure 1.1.4 Correlations of synaptic strength with other parameters. (Top) The degree of synaptic strength is proportional to the area of PSD, the numbers of AMPAR and the synaptic vesicles. (Bottom) Spine volume can be an indicator of synaptic strength, as well as the PSD area and docked synaptic vesicles in the presynaptic site. [Figure modified with permission from(Holtmaat \& Svoboda, 2009)] 


\subsubsection{The roles of actin filaments}

Actin filaments are one of the major proteins within living cells (Harvey et al, 2000) and play important roles in maintaining cellular shape and mobility (Pollard \& Cooper, 2009), in cellular morphogenesis (Maletic-Savatic et al, 1999; Tada \& Sheng, 2006) and in intracellular protein transport (Dillon \& Goda, 2005). Bundles of actin filaments in the leading edge of the cell called stress fibers can easily be seen under a conventional fluorescence light microscope with appropriate immune-staining. However, the individual actin filament is hardly visible by normal fluorescence microscopy because it is smaller than what can be resolved considering the optical diffraction limit.

(A)

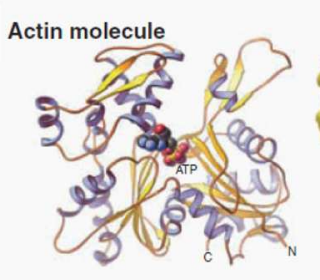

(B)

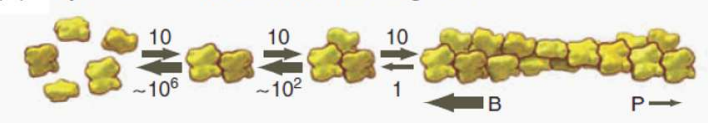

(C)

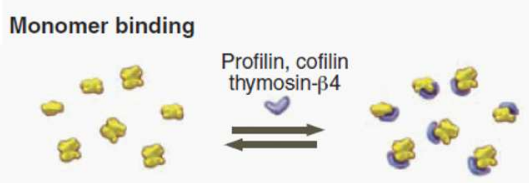

(D) Nucleation and elongation by formin

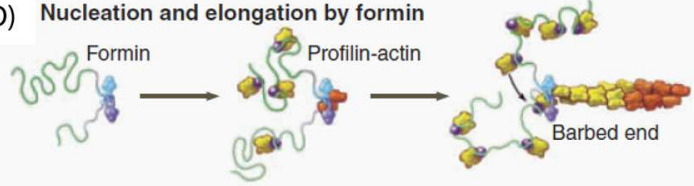

(E) Branching nucleation by Arp $2 / 3$ complex

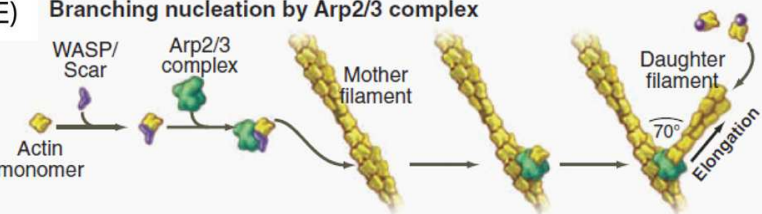

(F)
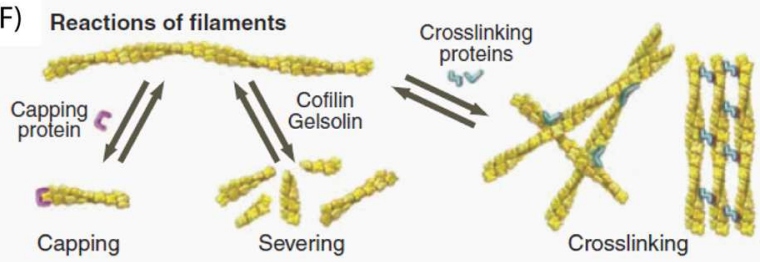

(G) Myosin-V walking on an actin filament
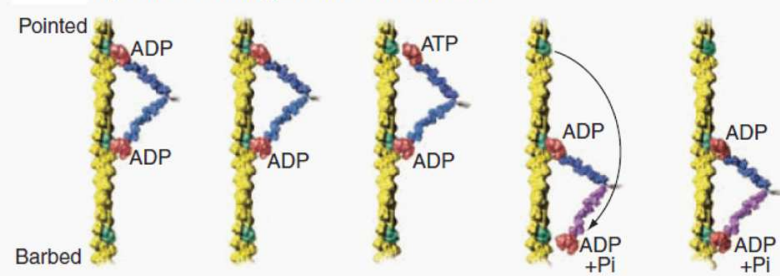

Figure 1.1.5 Structures of actin compositions and their functionalities: (A) The models of actin monomer (B) Nucleation and elongation of actin filament (C) Actin monomer binding proteins (D) Nucleation and elongation via Formin and Profilin (E) Arp2/3 protein responsible for filament branching (F) Capping, severing and cross linking proteins (G) Myosin motors walking along actin filament. [Figure modified with permission from (Pollard \& Cooper, 2009)] 
Actin filament polymers are composed of G-actin monomers. The polymer structure consists of a helical arrangement of monomers and has a diameter of 7-9 $\mathrm{nm}$. In most of the cells, actin serves as a dynamic organelle that exhibits equilibrium states of structural compositions between monomeric G-actin and filamentous F-actin (Pollard \& Borisy, 2003; Pollard \& Cooper, 2009). Remodeling and reorganization of actin filaments contributes to the function diversity of actin structure and enables sophisticated physiological processes within living cells (as shown in Figure 1.1.5). One of the fundamental functions of the dendritic spine in the postsynaptic area is to compartmentalize as a single unit to process and respond to unique signal transduction pathways rapidly and specifically. The processes of LTP and LTD will result in morphological changes of the dendritic spines, which is probably the reason why the actin structure is abundant within dendritic spines (Fischer et al, 1998; Honkura et al, 2008; Matsuzaki et al, 2004; Star et al, 2002; Tatavarty et al, 2009; Urban et al, 2011). Obviously, there are strong connections between actin dynamics, spine morphology and synaptic transmission strength. As mentioned in the last section, the size of PSD, the volume of spine head and the content of AMPAR and NMDAR can be used as readouts to judge the degree of potentiation. Clearly, actin filaments play a very important role in the changes in morphology as well as in the rapid transport of receptors into the membrane. Hence, it is of great importance to investigate actin dynamics with the function of synaptic plasticity to see how actin filaments govern and coordinate this activity-dependent process.

One of the biophysical parameters to quantify of actin dynamics is the turnover rate of actin filaments within living cells, which is often characterized by fluorescence recovery after photobleaching (FRAP). These studies showed that actin filaments undergo highly dynamic processes within the dendritic spine (Star et al, 2002). Electron micrographs demonstrate that the static actin filament structure in postsynaptic dendritic spines is composed of highly branched short form actin filaments (Korobova \& Svitkina, 2010). However, it is still surprisingly unclear how the actin filaments coordinate with each other to orchestrate downstream signal transduction, which later results in morphological plasticity. Several advanced methods, such as using stimulated emission depletion (STED) microscopy (Ding et al, 2009; Nagerl et al, 2008; Urban et al, 2011), fluorescence resonance energy transfer (FRET) (Okamoto et al, 2004) or photoactivated localization microscopy (PALM) (Frost et al, 2010; Honkura et al, 2008), provided very useful insights into the regulation of actin dynamics within dendritic spines, but in general, the lack of high temporal resolution limits the comprehensive understanding of fast actin dynamics within single dendritic spine during LTP. 


\subsection{Instrumental background}

The method developed and used in this research required the build-up of a wide-field fluorescence microscope (FM) for imaging fluorescently labeled biological samples and the combination with time-resolved fluorescence correlation spectroscopy (FCS) for monitoring the dynamics of other fluorescently labeled proteins within the biological sample. The aim of this study was to observe the overall living cell structure while simultaneously investigating dynamic processes on a (bio)-molecular level at very distinct and selected spots.

In the following sections, we provide some background and features focusing on fluorescence, the introduction of time-resolved techniques and the applications of microscopy techniques. Moreover, comparison of some similar techniques, such as fluorescence recovery after photobleaching (FRAP) and single particle tracking (SPT) which are usually employed to measure molecular dynamics will also be briefly described in this section.

\subsubsection{The principle of fluorescence}

Fluorescence techniques are employed universally in the biological sciences. Fluorescence is the emission of photons from fluorophores after absorbing photons from an excitation light source. The basic physics underlying this is as follows, during the short timescales of instantaneous light absorption $\left(\sim 10^{-15} \mathrm{~s}\right)$, an electron is promoted to a molecular orbital higher in energy. The new electronic configuration is termed as an electronic excited state $\left(S_{n}, n \geq 1\right)$, whereas the original configuration is the ground state $S_{0}$. Electronically excited molecules return to the ground state via various processes such as fluorescence emission, internal conversion and intersystem crossing potentially followed by phosphorescence.

A Jablonski diagram summarizes these processes and is shown in Figure1.2.1. The straight arrows depict all radiative processes that include photon absorption and emission, such as fluorescence emission. Energy dissipation without light emission is termed as nonradiative processes, as represented by curly arrows, which include internal conversion and intersystem crossing. Due to the difference in equilibrium nuclear structure, typically, a molecule is excited to higher vibrational level of electronic excited state upon light absorption. In the condensed phase, such as in aqueous solutions, the excited molecules relax quickly 
(within a time on the order of $\sim 10^{-12} \mathrm{~s}$ or less) to the lowest vibrational level of the first excited state (e.g. from $S_{1}, v^{\prime} \geq 1$ to $S_{1}, v^{\prime}=0$ ). This process is called vibrational relaxation.

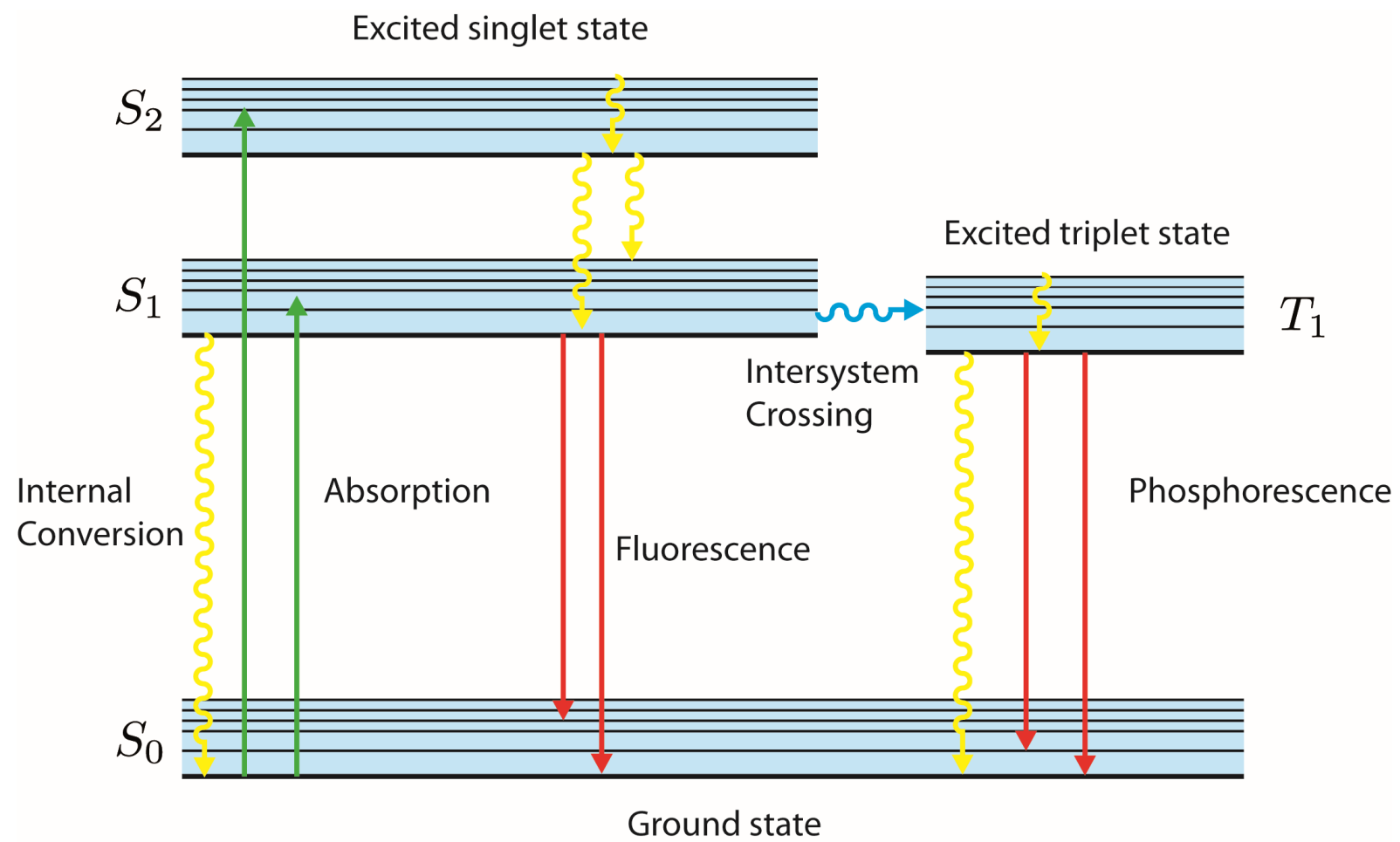

Figure 1.2.1 Jablonski diagram. A Jablonski diagram explains the energy states of a molecule. After the absorption of a photon, the molecule is promoted from the ground state $S_{0}$ and then either radiative (straight lines) or non-radiative (curly lines) process brings the molecule to lower energy states.

Table 1.1 Comparisons of the timescale of various molecular reactions with regard to absorption, non-radiative processes and emission.

\begin{tabular}{|c|c|}
\hline Type & Time scale (seconds) \\
\hline Absorption & $\sim 10^{-15}$ (instantaneous) \\
\hline $\begin{array}{c}\text { Internal conversion } \\
\text { Molecular relaxation }\end{array}$ & $\sim 10^{-12}$ \\
\hline Fluorescence & $\sim 10^{-9}$ \\
\hline Phosphorescence & $\sim 10^{-3}-10^{2}$ \\
\hline
\end{tabular}


Upon rapid molecular relaxation to the lowest vibrational level of the first electronic excited level $S_{1}\left(v^{\prime}=0\right)$, fluorescence occurs as depicted in red straight arrows in Figure 1.2.1. Due to energy losses from vibrational relaxation, the energy of the emitted photons is typically lower than that of the absorbed photons, which implies that the wavelength of the emission light is longer than that of the excitation. This energy difference between absorption and emission is called Stokes shift.

Besides fluorescent emission, there are other deactivation pathways such as the nonradiative internal conversion. Internal conversion occurs typically more efficiently while the energy gap between $S_{1}$ and $S_{0}$ is smaller. Molecules cross to the overlapping higher vibrational levels of $\mathrm{S}_{0}$ more easily when the gap is smaller, and the energy is lost owing to subsequent vibrational relaxation. There is also the probability that triplet states of the fluorophore can be populated due to a process called intersystem crossing, i.e. from $S_{1}$ to $T_{1}$ (the curly blue arrow in Figure 1.2.1). Emission from triplet states is referred to as phosphorescence.

For all radiative and non-radiative processes, rate constants can be defined to quantify the probability that the corresponding processes take place. The rate constants of fluorescence emission, internal conversion and intersystem crossing typically are denoted by $\Gamma, \mathrm{k}_{\mathrm{IC}}$ and $\mathrm{k}_{\mathrm{ISC}}$, respectively. Another important parameter is the fluorescence quantum yield (equation 1.1 and 1.2) that can be defined as the number of emitted fluorescence photons divided by the number of photons previously absorbed in a large number of excitation and relaxation cycles.

$$
\begin{aligned}
& Q=\frac{\text { Number of emitted fluorescence photons }}{\text { Number of absorbed photons }} \\
& Q=\frac{\Gamma}{\Gamma+\mathrm{k}_{\mathrm{IC}}^{S_{1}-S_{0}}+\mathrm{k}_{\mathrm{ISC}}}
\end{aligned}
$$


Combining the rate constants of non-radiative parts, $\mathrm{k}_{\mathrm{IC}}^{\mathrm{S} 1-\mathrm{S} 0}$ and $\mathrm{k}_{\mathrm{ISC}}$, the formula of fluorescence quantum yield can be further simplified as:

$$
Q=\frac{\Gamma}{\Gamma+\mathrm{k}_{\mathrm{nr}}}
$$

From the equation above, it can be seen that smaller non-radiative rates result in higher fluorescence quantum yields. If a molecule emits without any non-radiative decay loss, the maximum quantum yield could theoretically reach $Q=1$. The lifetime of the excited state, $\tau$, characterizes the average time that an excited fluorophore stays in this state. The excited state population at a given time $t$ is defined as $\mathrm{N}(\mathrm{t})$, and decreases with the decay rate $(\Gamma+\mathrm{knr})$ which is inversely proportional to the life time $\tau$ when neglecting intersystem crossing effect. The relationship between time dependent changes of $\mathrm{N}(\mathrm{t})$ and the decay rate is given by equation (1.4):

$$
\frac{d N(t)}{d t}=-\left(\Gamma+\mathrm{k}_{\mathrm{nr}}\right) N(t)
$$

Integrating once, we obtain:

$$
\begin{aligned}
& N(t)=N_{0} e^{-t / \tau} \\
& \tau=\frac{1}{\Gamma+\mathrm{k}_{\mathrm{nr}}}
\end{aligned}
$$

According to equation (1.5), the population of excited molecules will decay exponentially as a function of time, where $\mathrm{N}_{0}$ represents the initial population in $\mathrm{S}_{1}$ state after energy absorption. In fluorescence experiments, the emission intensity $I(t)$ is recorded instead of the number of molecules. However, the emission intensity is proportional to $N(t)$ (Lakowicz, 2006). As a result, the fluorescence intensity as a function of time, I(t), can be written again as shown in equation (1.7), where $\mathrm{I}_{0}$ represents initial fluorescence intensity, which also follows the rule of exponential decay with a time constant $\tau$.

$$
I(t)=I_{0} e^{-t / \tau}
$$


The absorption probability of OPE $\left(P_{a b s}^{O P E}\right)$ is linearly proportional to the excitation intensity $I$ (equation 1.8). On the other hand, nonlinear excitation such as two-photon excitation (TPE) is also possible. A simplified Jablonski diagram of TPE is also shown in Figure 1.2.3. This process involves the simultaneous absorption of two photons by a molecule. The probability $\left(P_{a b s}^{T P E}\right)$ to excite molecules is proportional to the square of excitation light intensity (equation 1.9) in TPE. It can be conveniently achieved by using femtosecond pulse lasers because of its high peak intensities compared to continuous mode laser.

$$
\begin{aligned}
& P_{\mathrm{abs}}^{\mathrm{OPE}} \propto I \\
& P_{\mathrm{abs}}^{\mathrm{TPE}} \propto I^{2}
\end{aligned}
$$




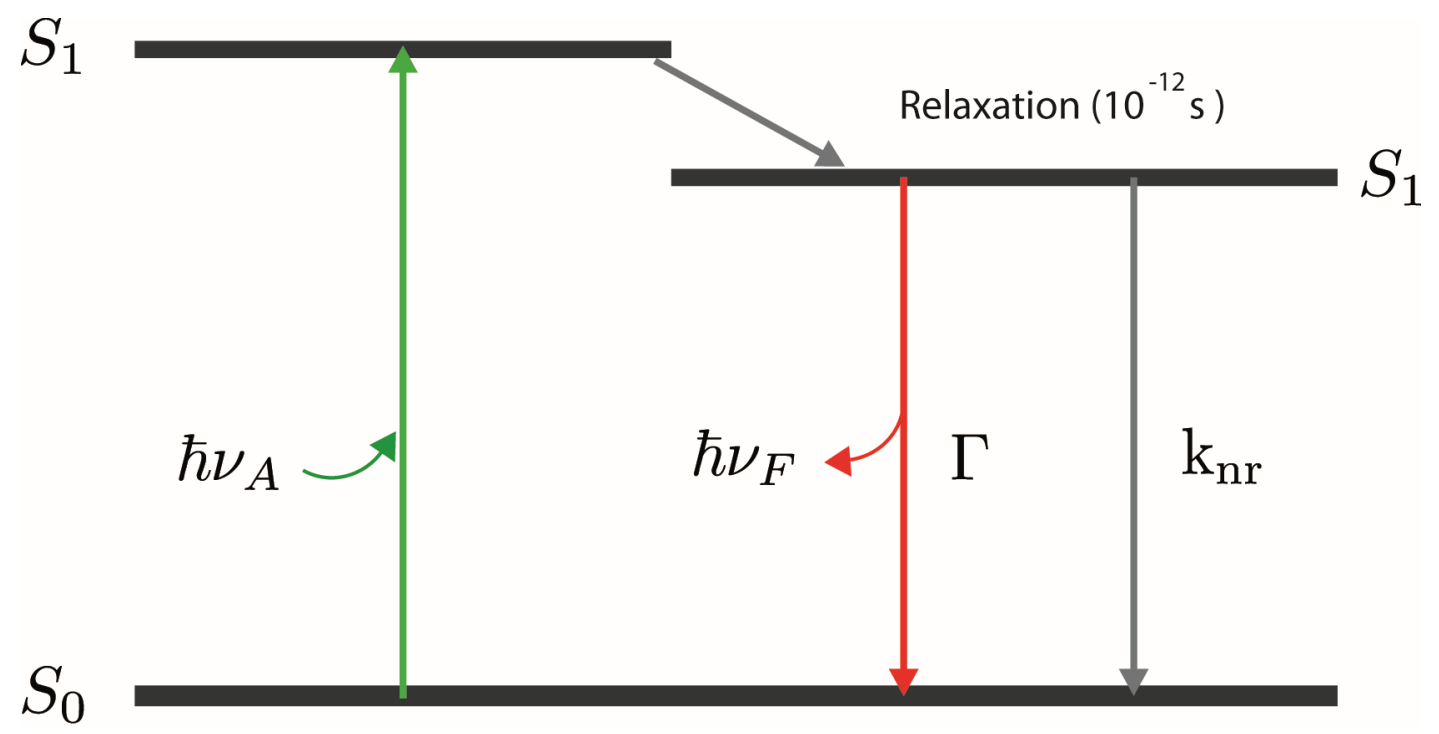

Figure 1.2.2 Illustration of fluorescence quantum yield and lifetime. A simplified version of the Jablonski diagram is shown to explain the quantum yield and fluorescence lifetime, where $\Gamma$ and $\mathrm{k}_{\mathrm{nr}}$ represent the rate constants of radiative part and non-radiative part, respectively.

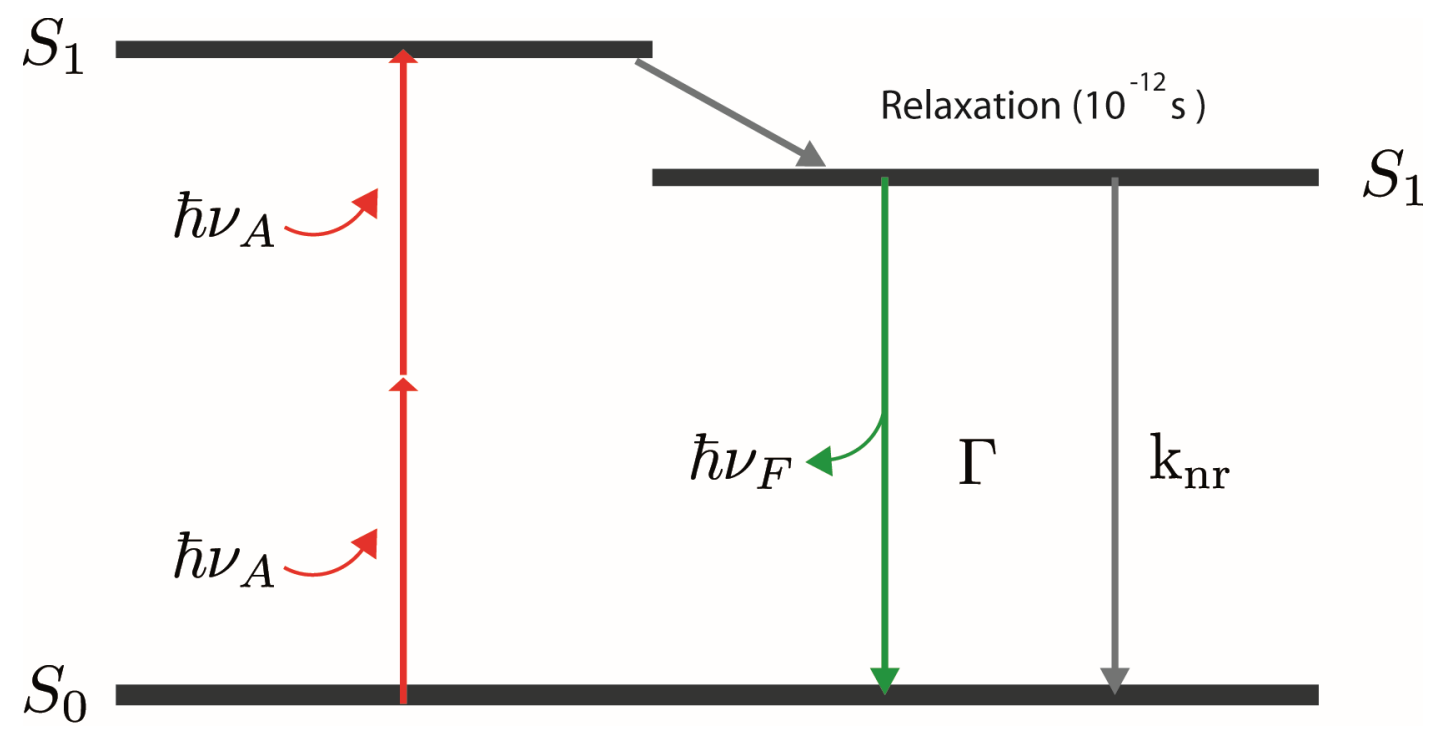

Figure 1.2.3 Simplified Jablonski diagram to explain two-photon excitation. With two simultaneous photon absorptions in the focal spot, the emission of fluorescence is also possible. 


\subsubsection{Fluorescence correlation spectroscopy (FCS)}

The theory and initial experiments of fluorescence correlation spectroscopy (FCS) were developed in the 1970s (Magde et al, 1972; Magde et al, 1974). FCS can be applied for elucidating molecular dynamics or interactions within a living cell (Kim et al, 2007; Schwille et al, 1999), such as protein interactions or diffusion mobility (Rusu et al, 2004; Weiss et al, 2003).

The principle behind FCS measurements involves analyzing recorded intensity fluctuations from fluorescence molecules within tiny focal volume at sub-micron level. From FCS analysis, parameters like average particle numbers, diffusion mobility and even particle brightness can be determined. The observed intensity fluctuations can be due to the translational diffusion (Ehrenberg \& Rigler, 1974; Tsay et al, 2006), the molecular interactions (Brazda et al, 2011; Gowrishankar et al, 2012; Mueller et al, 2011; Sezgin et al, 2012) or the fluorescence resonance energy transfer effect (Cypionka et al, 2009; Nath et al, 2010; Price et al, 2011; Remaut et al, 2005).

In the course of this work, autocorrelation is used to analyze actin dynamics within living neuron cells. Autocorrelation provides information about self-similarities in the fluorescence intensity fluctuations. Autocorrelation curves are generated by correlating the recorded intensity fluctuation curve $\mathrm{I}(\mathrm{t})$ with itself with respect to a variable correlation time $\tau$ $(\mathrm{I}(\mathrm{t}+\tau))$. A simple autocorrelation function is shown in equation (1.10) where $\mathrm{T}$ stands for data acquisition time. The generation of autocorrelation curve is schematically shown in Figure 1.2.4. The physical parameters, such as particle numbers or diffusion mobility, can be derived by fitting the autocorrelation curves with an appropriate model.

$$
G(\tau)=\frac{1}{T} \int_{0}^{T} I(t) I(t+\tau) d t
$$




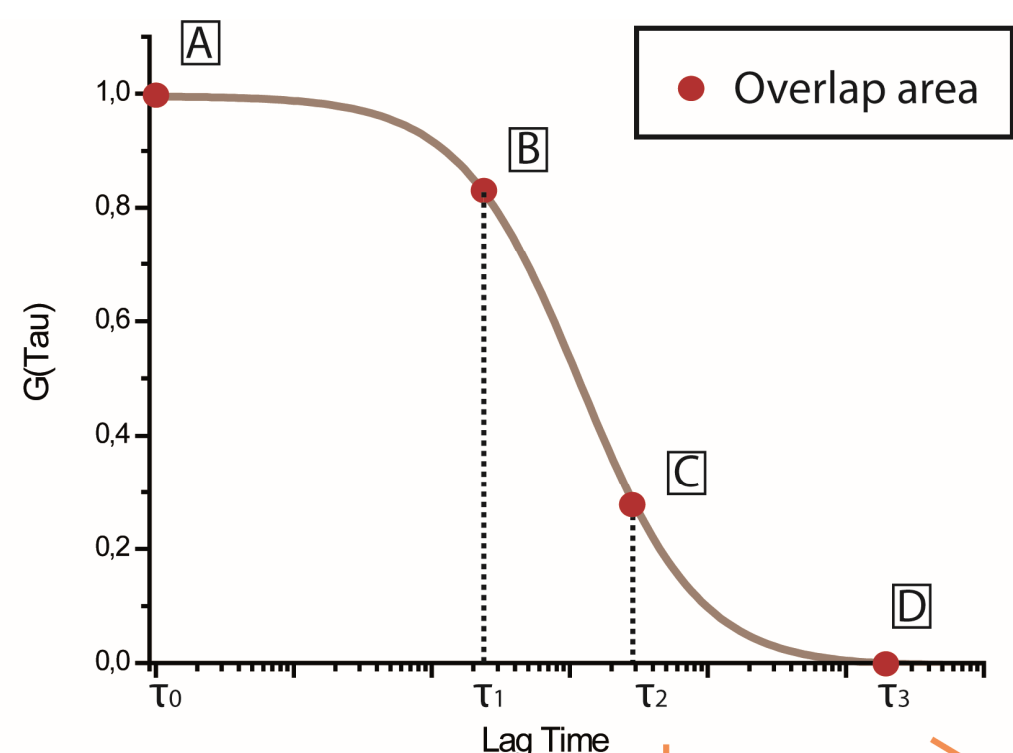

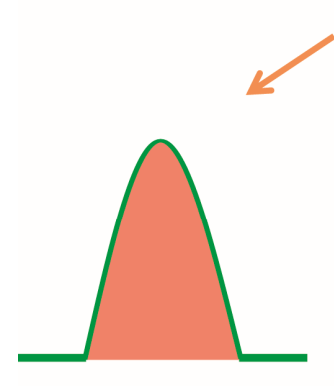

$A: I(t) \mid\left(t+t_{0}\right)$

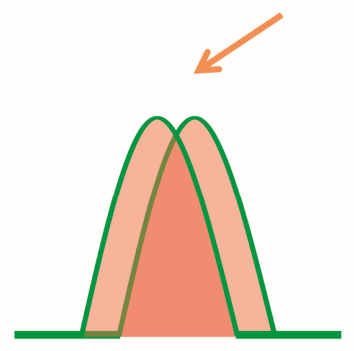

$B: I(t) \mid\left(t+\tau_{1}\right)$
Lag Time
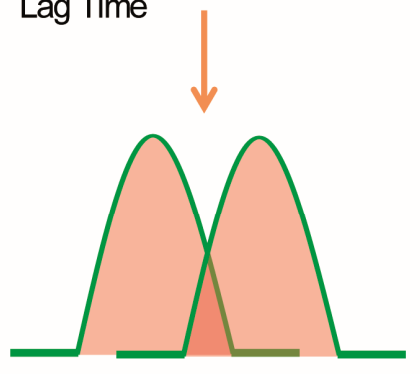

$C: I(t) \mid\left(t+\tau_{2}\right)$
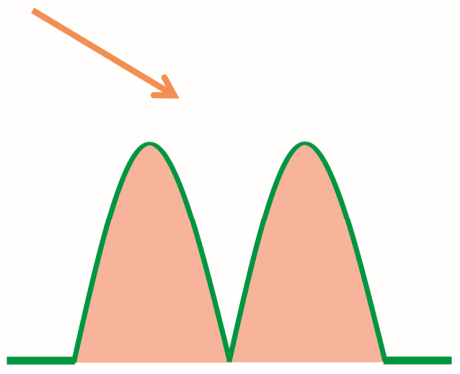

$D: I(t) \mid\left(t+\tau_{3}\right)$

Figure 1.2.4 Principle of autocorrelation analysis. An autocorrelation curve can be derived from the results of the overlapping areas by shifting the original fluorescence intensity with increasing correlation times $\left(\tau_{0}, \tau_{1}, \tau_{2}\right.$ and so on...). The overlapping area is in its maximum in position $\mathbf{A}$ and normalized to unity at correlation time zero. With the increase of correlation time, from position $\mathbf{B}$ to $\mathbf{D}$, the overlapping becomes smaller and reaches a value of zero after the long correlation time shifting. 
The autocorrelation function used in this is thesis is shown in equation (1.11). It is assumed that there are fluctuation signals resulting from the changes of local concentration $\delta \mathrm{C}$ within the effective volume $V_{\text {eff. }}$ These fluctuations around the mean value are defined by equation (1.12). The fluctuations caused by fluctuations of local concentration can also be written as equation (1.13) (Heinze et al, 2000).

$$
\begin{aligned}
& G(\tau)=\frac{\langle I(t) I(t+\tau)>}{<I(t)>^{2}}-1=\frac{\langle\delta I(t) \delta I(t+\tau)>}{<I(t)>^{2}} \\
& \delta I(t)=I(t)-<I(t)> \\
& \delta I(t)=\kappa \int_{V} I_{e x}(\vec{r}) \cdot S(\vec{r}) \cdot \sigma \cdot q \cdot \delta C(\vec{r}, t) d V
\end{aligned}
$$

\section{Parameters:}

$\kappa:$ detection efficiency

$I_{e x}(\vec{r})$ : intensity distribution of excitation light source

$S(\vec{r})$ : optical transfer function of whole optical system

$\sigma$ : molecular absorption cross-section

$q:$ quantum yield of fluorephores

$\delta C(\vec{r}, t)$ : fluctuations of the local particle concentration at time $t$ 
The term " $\mathrm{Iex}_{\mathrm{ex}}(\vec{r}) \cdot \mathrm{S}(\vec{r})$ " can be interpreted as the probability of detecting fluorescence from a molecule at certain position and is related to the spatial distribution of the emitted light $\mathrm{W}(\vec{r})$. The distribution can be approximated by a Gaussian distribution (Rigler et al, 1993).

$$
I_{e x}(\vec{r}) \cdot S(\vec{r}) \propto W(\vec{r}) \approx e^{-2 \frac{x^{2}+y^{2}}{r_{0}^{2}}} \cdot e^{-2 \frac{z^{2}}{z_{0}^{2}}}
$$

We note that the distribution decays by a factor of $1 / \mathrm{e}^{2}$ at $\mathrm{r}_{0}$ in the lateral direction $(\mathrm{x}, \mathrm{y})$ and $\mathrm{z}=\mathrm{z}_{0}$ in axial direction in equation (1.14). The other parameters in equation (1.13) such as $\kappa, \sigma$ and $\mathrm{q}$ can be combined with $\mathrm{I}_{0}$, the maximum amplitude of $\mathrm{I}_{\mathrm{ex}}(\vec{r})$, to form a combined parameter called photon count rate per molecule per second (CPP) shown in equation (1.15), with which the signal-to-noise ratio of different measurements can be evaluated to compare the efficiency among different setups.

$$
\eta=I_{0} \cdot \kappa \cdot \sigma \cdot q
$$

With these simplifications and approximations, equation (1.13) can be re-written as (1.16). Hence, combining (1.11) and (1.16), we obtain the autocorrelation function in the form of (1.17). Assuming that the properties of the fluorophore are kept constant, and $\delta \eta$, the change rate of $\mathrm{CPP}$, is equal to zero, therefore, equation (1.17) further simplifies to equation (1.18).

$$
\begin{aligned}
& \delta I(t)=\int W(\vec{r}) \cdot \delta(\eta \cdot C(\vec{r}, t)) d V \\
& G(\tau)=\frac{\iint W(\vec{r}) W\left(\overrightarrow{r^{\prime}}\right)<\delta(\eta \cdot C(\vec{r}, t)) \cdot \delta\left(\eta \cdot C\left(\overrightarrow{r^{\prime}}, t+\tau\right)\right)>d V d V^{\prime}}{\left(\int W(\vec{r}) \cdot<\delta(\eta \cdot C(\vec{r}, t)>d V)^{2}\right.} \\
& G(\tau)=\frac{\iint W(\vec{r}) W\left(\overrightarrow{r^{\prime}}\right)<\delta C(\vec{r}, 0) \cdot \delta C\left(\overrightarrow{r^{\prime}}, \tau\right)>d V d V^{\prime}}{\left(<C>\int W(\vec{r}) d V\right)^{2}}
\end{aligned}
$$


For a three dimensional free diffusion process characterized by a diffusion coefficient $\mathrm{D}$, the number density autocorrelation term $\left\langle\delta \mathrm{C}(\vec{r}, 0) \delta \mathrm{C}\left(\overrightarrow{r^{\prime}}, \tau\right)\right\rangle$ can be calculated based on the equation (1.19) and (1.20), where $<\mathrm{C}>$ represents localized average particle concentration.

$$
\begin{aligned}
& \frac{\partial C(r, t)}{\partial t}=D \nabla^{2} C(r, t) \\
& <\delta C(\vec{r}, 0) \cdot \delta C\left(\overrightarrow{r^{\prime}}, \tau\right)>=<C>\frac{1}{(4 \pi D \tau)^{3 / 2}} e^{-\frac{\left(\vec{r}-\vec{r}^{\prime}\right)^{2}}{4 D \tau}}
\end{aligned}
$$

The autocorrelation function $\mathrm{G}(\tau)$ in equation (1.18) can be re-written by substituting with equation (1.14) and (1.20). The diffusion time $\tau_{\mathrm{D}}$ is related to the diffusion coefficient $\mathrm{D}$ via equation (1.22). The final autocorrelation function obtained by combining equations (1.21), (1.22) and (1.23) results in (1.24). From the experiment, the particle numbers within focal volume can be derived at correlation time zero (equation (1.25)).

$$
\begin{aligned}
& G(\tau)=\frac{1}{\pi^{3 / 2} r_{0}^{2} \cdot z_{0} \cdot\langle C>} \cdot \frac{1}{1+\frac{4 D \tau}{r_{0}^{2}}} \cdot \frac{1}{\sqrt{1+\frac{4 D \tau}{z_{0}^{2}}}} \\
& \tau_{D}=\frac{r_{0}^{2}}{4 D} \\
& V_{\text {eff }}=\pi^{3 / 2} \cdot r_{0}^{2} \cdot z_{0} \\
& G(\tau)=\frac{1}{V_{\text {eff }} \cdot<C>} \cdot \frac{1}{1+\frac{\tau}{\tau_{D}}} \cdot \frac{1}{\sqrt{1+\left(\frac{r_{0}}{z_{0}}\right)^{2} \cdot \frac{\tau}{\tau_{D}}}} \\
& G(0)=\frac{1}{V_{\text {eff }} \cdot\langle C>}=\frac{1}{N}
\end{aligned}
$$




\subsubsection{Focal volume (OPE and TPE) and diffusion coefficient}

A well-calibrated focal volume is a key factor in the accurate determination of diffusion coefficients. The absorption probabilities of a photon under OPE and TPE are proportional to the intensity (I) and the square of intensity $\left(\mathrm{I}^{2}\right)$, respectively.

Because of the inherent non-linearity of two-photon excitation, the appropriate focal volume depends on the square of the intensity point spread function (IPSF${ }^{2}$ ), as shown in Figure 1.2.5. The probability to detect a fluorescence signal emitted from certain position is related to excitation light source and overall optical transfer function, and can be calculated approximately as a three-dimensional Gaussian function as shown in Figure 1.2.5(B). The effective focal volume is calculated by computing the scale for the intensity to reduce by a factor of 1/e $\mathrm{e}^{2}$ (Heinze et al, 2000; Schwille et al, 1999). Hence, the focal volumes of both OPE and TPE can be written as equation (1.26) and (1.27).

$$
\begin{aligned}
& V_{\mathrm{eff}}^{\mathrm{OPE}}=\pi^{3 / 2} \cdot r_{0}^{2} \cdot z_{0} \\
& V_{\mathrm{eff}}^{\mathrm{TPE}}=(\pi / 2)^{3 / 2} \cdot r_{0}^{2} \cdot z_{0} \\
& \tau_{D}^{\mathrm{TPE}}=\frac{r_{0}^{2}}{8 D} \\
& V_{\mathrm{eff}}^{\mathrm{TPE}}=\left(4 \pi \cdot \tau_{D}^{\mathrm{TPE}} \cdot D\right)^{3 / 2} \cdot \omega
\end{aligned}
$$

Comparing the focal volumes between OPE and TPE, one can find that the total volume under TPE decreased by $\sqrt{8}$, which means the effective focal volume in each direction decreased by a factor of $\sqrt{2}$. With dimension of focal volume decreasing, the diffusion time under TPE also changes by a factor of 2. Compared with equation (1.22), equation (1.28) represents the diffusion time under TPE. 
The determination of experimental focal volume can be characterized by measuring the diffusion time of Rhodamine 6G (R6G) in the FCS setup. With averaged diffusion time $\tau_{\mathrm{D}}=(4.6 \pm 0.14) \cdot 10^{-5} \mathrm{~s}$ and for the structural parameter $\omega=z_{0} / r_{0} \cong 5$ in this setup, one can substitute the parameters with those numbers in equation (1.29). According to the well characterized diffusion coefficient of R6G: $D_{\mathrm{R} 6 \mathrm{G}}=4.41 \times 10^{-6} \mathrm{~cm}^{2} / \mathrm{s}$ (Culbertson et al, 2002; Gendron et al, 2008; Müller et al, 2008), the experimental TPE focal volume in this setup can be calculated as about $0.6 \times 10^{-18} \mathrm{~m}^{3}$, that is about $0.6 \mathrm{fL}$. Based on the optical property of TPE, the focal volume can therefore reach down to the scale of sub-femtoliter $(\sim \mathrm{fL})$ in a pinholefree set-up. 
(A)

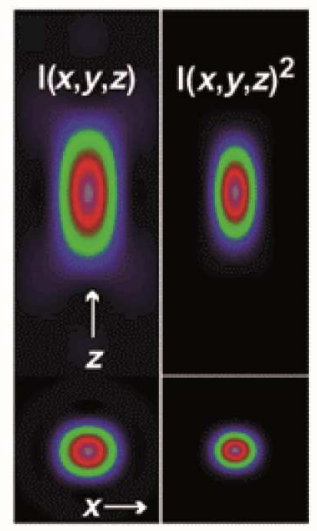

(B)

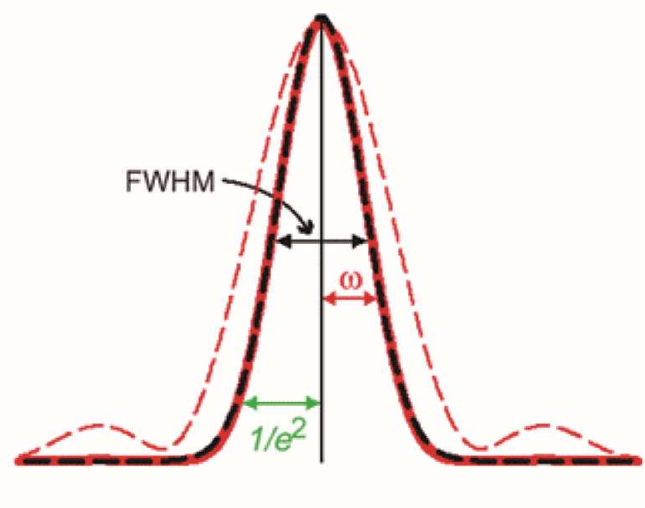

(C)

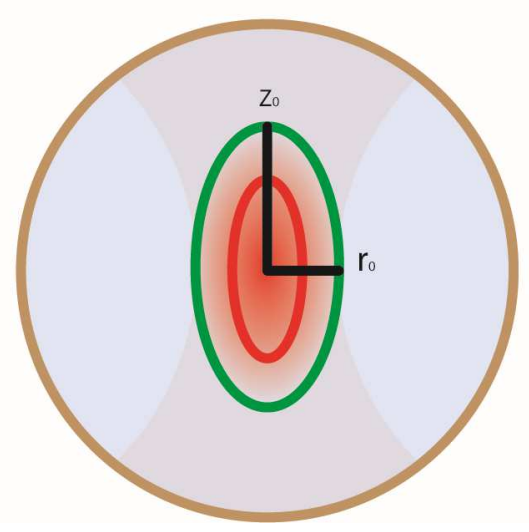

Figure 1.2.5 Intensity point spread function (IPSF) of one-photon excitation (OPE) and two-photon (TPE). (A) The comparison of the IPSF of OPE and TPE (B) Approximately 3D Gaussian distribution fitting of light intensity, or more precisely described by Lortentz distribution (Dertinger et al, 2007), Figure modified from (Zipfel et al, 2003) (C) Brief illustration of focal volume of OPE and TPE; the focal volume decreased by $\sqrt{8}$ while TPE is applied. 


\subsubsection{Fluorescence Microscopy (FM)}

By taking advantages of fluorescence labeling within living cells, fluorescence microscopy has long been employed as a tool to investigate cellular function in vivo or in vitro (Baumgartel et al, 2012; Germain et al, 2012; Toomre, 2012). There are several basic components to construct a set of fluorescence microscopy. For the most common used fluorescence microscopy, such as wide-field fluorescence microscopy, the key components include: a light source to excite the fluorophores, a dichroic mirror to reflect and transmit light for excitation and observation, an optical filter to selectively choose appropriate signals and a detector to record the signal.

Excitation of a large sample area is possible by focusing the incident light on the back aperture of the microscope's objective lens. As shown in Figure 1.2.6, this is a schematic of wide-field fluorescence microscopy setup. Two-dimensional large-area detectors such as charge-coupled devices (CCD) are often used to record the fluorescence images.

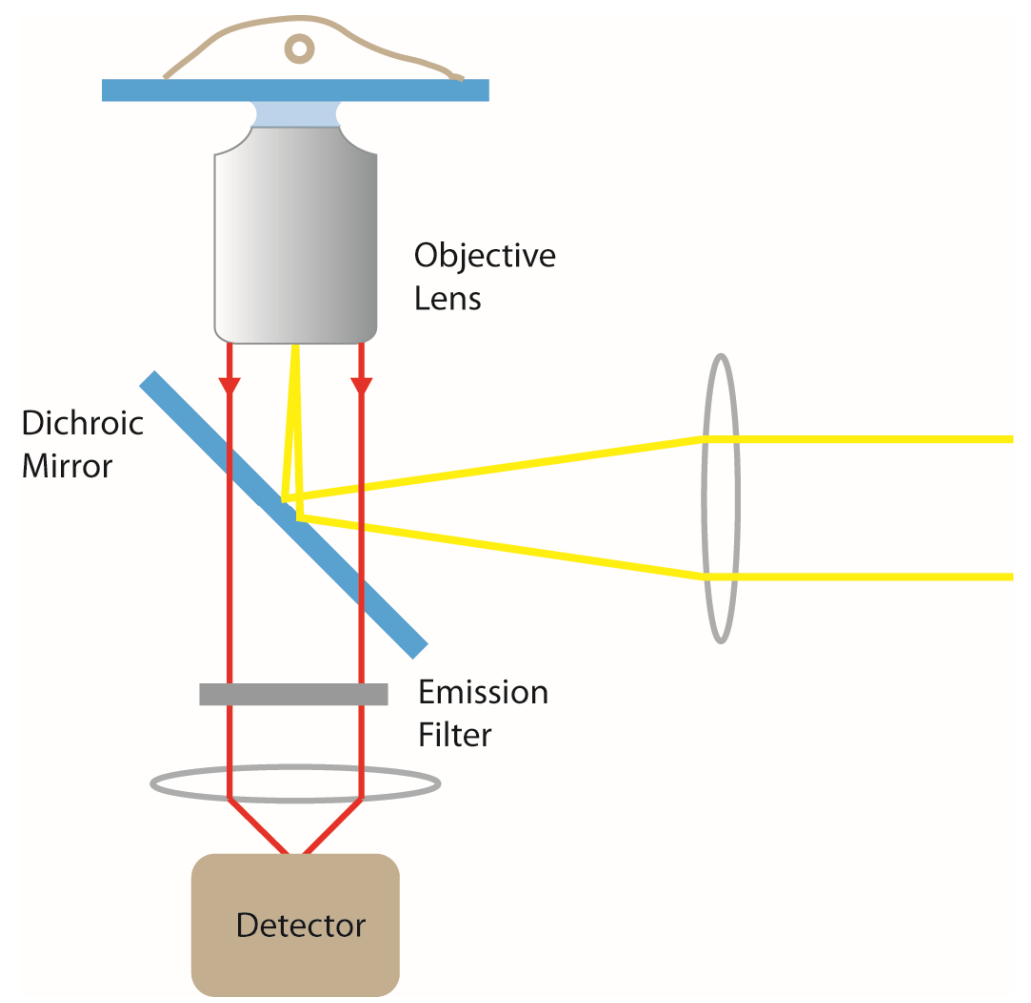

Figure 1.2.6 Schematic representation of a wide-field fluorescence microscope setup. A wide-field fluorescence microscope consists of: an objective lens, a dichroic mirror, an emission filter and a detector. With the light focusing on the back aperture of the lens, the wide-field fluorescence microscope can be made to observe fluorescence images in large areas. 


\subsubsection{Other methods to measure molecular diffusion}

Molecular diffusion is essential for maintaining cellular functions. It is therefore important to quantitatively decipher the dynamic behaviors of these molecules. Besides FCS measurements, there are other optical platforms that are suitable for studying the molecular dynamics within living cells, such as fluorescence recovery after photobleaching (FRAP) (Axelrod et al, 1976; Schlessinger et al, 1976) and single particle tracking (SPT) (Kusumi et al, 1993; Qian et al, 1991).

FRAP, as shown in Figure 1.2.7(A), is a fluorescence technique that takes advantage of irreversible photochemical bleaching. First, fluorescence molecules are bleached within a region of interest by using intense laser irradiation. After bleaching, the excitation light source is applied to image the bleached area and thus making the intensity recovery after photobleaching recordable by a detector. Molecules labeled with fluorescence dyes start to diffuse until a steady state is reached. Because of this dynamic mobility of diffusing molecules, the intensity recovers to a certain level. Based on this property, FRAP can be used to distinguish and to determine mobile/immobile molecules within living cells. Furthermore, one can also interpret the diffusion constant by fitting the intensity recovery curve into proper mathematical models. The recovery efficiency relies on several parameters, such as the geometric property of bleached area, the dynamic behavior of bleached molecules and even the binding affinity of different molecules. The quantitative information derived from FRAP techniques, however, is an averaging outcome of many particles.

The method of SPT, as shown in Figure 1.2.7(B), is to record and analyze the trajectories of small fluorescent particles that are tagged in a target protein at high spatial and temporal resolution by making use of a sensitive detector. First of all, the fluorescence molecules attach to the molecules of interest and the mobile behavior is recorded as a function of time. By using a mathematical approach to calculate the central positions of each moving particle, one can determine the so-called "mode of motion" from the mean square displacement of the particle trajectories. The curve of mean square displacement (MSD) is a straight line (MSD $\sim \mathrm{D} t$, where $\mathrm{D}$ : diffusion coefficient, $t$ : observation time) if the molecules move as a three dimensional Brownian diffusion, as shown in Figure 1.2.7 (B), right side, blue curve. The diffusion coefficient can also be derived from the slope. Other diffusion modes can also be monitored, such as hindered diffusion (green curve) and diffusion behavior that mixed with flow phenomena (red curve). The modes of diffusion provide not only the 
dynamic properties of the monitored molecules but also give information concerning the surrounding structure composition as well as the information of their interacting molecules.
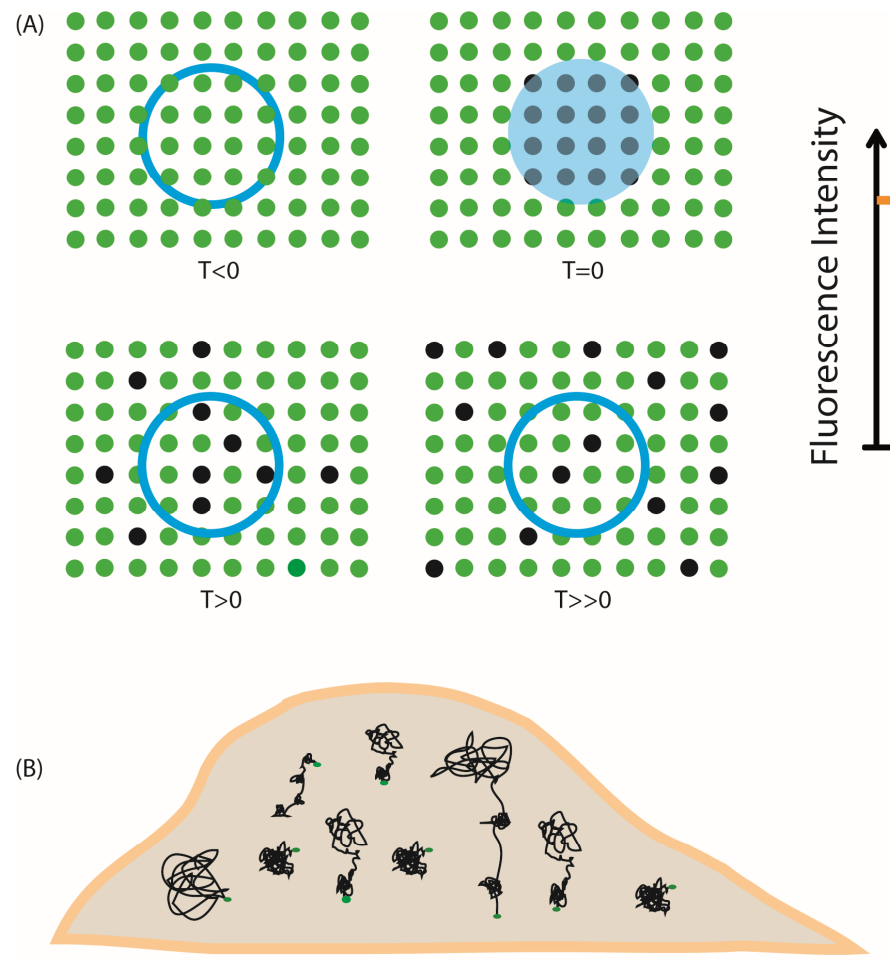
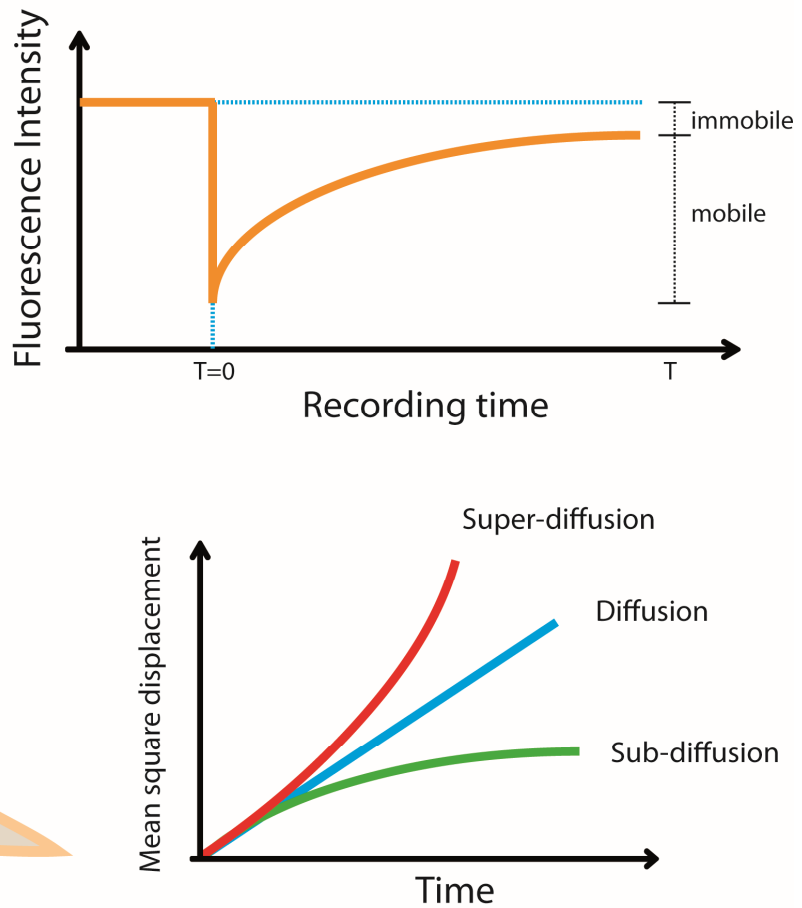

Figure 1.2.7 Other methods for molecular diffusion measurements: (A) Fluorescence recovery after photobleaching (FRAP) can provide the averaging diffusion dynamics of many molecules. (B) Single particle tracking provides the information on diffusion modes. Blue, green and red curves represent 3D Brownian diffusion, hindered diffusion and active transport mode, respectively. 


\subsection{Outline of this study}

In the content of my thesis, the construction and calibration of a setup that combined conventional wide field fluorescence microscopy (FM) and two-photon fluorescence correlation spectroscopy (2P-FCS) was first described. The combination of FM and 2P-FCS allowed the investigation of the actin dynamic changes at higher temporal resolution and to gain better insights into the dynamic behavior of the different forms of actin filamentous structures. Samples investigated in the course of this study were living neuronal cells (CA3) that had been transfected with eGFP-actin and fCherry to simultaneously monitor changes in actin dynamics and morphology with the function of Tetraethylammonium (TEA) induced LTP. The actin dynamics before and after TEA stimulation was scrutinized to correlate with the morphological plasticity after long-term potentiation (LTP).

Using FCS measurement, we can distinguish two groups of actin filaments within living cell by two-component fitting models. Further, the absolute particle number and brightness can be compared before and after TEA stimulation. We conclude that dendritic spines underwent morphological enlargement with the function of TEA stimulation are correlated to dynamic changes of actin filaments. These more dynamic actin filaments serve as an important role to drive morphological enlargement after LTP. 


\section{Materials and Methods}

\subsection{Materials}

\subsubsection{Chemicals}

All the chemicals used in this research were purchased from those companies: SigmaAldrich (St. Louis, USA), Merck (Darmstadt, Germany), BIOTREND Chemicals AG (Zurich, Switzerland), Invitrogen (USA), Molecular Probes (USA), Fluka (Switzerland)

\subsubsection{Preparation of organotypic hippocampus slices}

Hippocampal organotypic cultures were prepared from postnatal day 5 (P5) C57BL/6 wild-type mice as described in reference (Stoppini et al, 1991). The hippocampi were dissected in ice-cold sterile Gey's balanced salt solution (GBSS) and sliced transversally at a thickness of $400 \mu \mathrm{m}$ using a McIllwain tissue chopper. The slices were placed on Millicells $\mathrm{CM}$ membrane inserts (Millipore) and cultivated at $37^{\circ} \mathrm{C}, 5 \% \mathrm{CO}_{2}, 99 \%$ humidity, with a medium containing 50\% BME (Eagle, with Hank's salts without glutamine), 25\% Hank's buffered salt solution (HBSS), $1 \mathrm{ml}$ of glucose (50\%), 25\% donor equine serum (HyClone), and $0.5 \mathrm{ml}$ of L-glutamine ( $200 \mathrm{mM}$ stock solution). To reduce the number of non-neuronal cells, a mixture of antimitotic drugs (cytosine arabinoside, uridine, and fluorodeoxyuridine; $10^{-6}-10^{-7} \mathrm{M}$ each; Sigma-Aldrich) was applied for $24 \mathrm{~h}$. The preparation of hippocampal organotypic cultures was done by our collaborators from TU-Braunschweig.

\subsubsection{MCF-7 cancer cells}

MCF-7 cells (human breast adenocarcinoma cell line) were cultured by using Eagle's minimum essential medium (MEM), which is supplemented with $10 \%$ fetal bovine serum, $1 \%$ penicillin/streptomycin. One million cells in $10 \mathrm{ml}$ MEM medium were seeded in a $10 \mathrm{~cm}$ petri dish with 2 or 3 cover slips. Actin filaments were expressed with eGFP for FCS observation and the membrane was stained by PKH26 (Sigma-Aldrich) red fluorescence dye 
for the morphological observation in FM. This cell was provided by Dr. Han-Ning Chuang from Universitat Klinikum Goettingen.

\subsubsection{Buffers}

The buffers used within this research are listed as follows (Gu et al, 2010; RothAlpermann et al, 2006):

Table 2.1 Buffers

\begin{tabular}{|c|c|c|c|}
\hline \multicolumn{4}{|c|}{ Physiological condition buffer for cells } \\
\hline Buffer & Concentration & Components & M.W. (g/mole) \\
\hline \multirow{6}{*}{$\begin{array}{l}\text { HEPES Buffer } \\
\qquad(\mathrm{pH}=7.4)\end{array}$} & $140 \mathrm{mM}$ & $\mathrm{NaCl}$ & 58.44 \\
\hline & $5 \mathrm{mM}$ & $\mathrm{KCl}$ & 74.55 \\
\hline & $2 \mathrm{mM}$ & $\mathrm{CaCl}_{2} \cdot 2 \mathrm{H}_{2} \mathrm{O}$ & 147.02 \\
\hline & $1.5 \mathrm{mM}$ & $\mathrm{MgCl}_{2} \cdot 6 \mathrm{H}_{2} \mathrm{O}$ & 203.3 \\
\hline & $10 \mathrm{mM}$ & Glucose & 180.16 \\
\hline & $25 \mathrm{mM}$ & HEPES & 238.31 \\
\hline
\end{tabular}

\begin{tabular}{|c|c|c|c|}
\hline \multicolumn{4}{|c|}{ LTP induction buffer for neuron cells } \\
\hline Buffer & Concentration & Components & M.W. (g/mole) \\
\hline \multirow{7}{*}{$\begin{array}{c}\text { HEPES } \\
\text { Buffer } \\
\text { With TEA } \\
(\mathrm{pH}=7.4)\end{array}$} & $140 \mathrm{mM}$ & $\mathrm{NaCl}$ & 58.44 \\
\hline & $5 \mathrm{mM}$ & $\mathrm{KCl}$ & 74.55 \\
\hline & $5 \mathrm{mM}$ & $\mathrm{CaCl}_{2} \cdot 2 \mathrm{H}_{2} \mathrm{O}$ & 147.02 \\
\hline & $0.1 \mathrm{mM}$ & $\mathrm{MgCl}_{2} \cdot 6 \mathrm{H}_{2} \mathrm{O}$ & 203.3 \\
\hline & $10 \mathrm{mM}$ & Glucose & 180.16 \\
\hline & $25 \mathrm{mM}$ & HEPES & 238.31 \\
\hline & $25 \mathrm{mM}$ & $\begin{array}{l}\text { Tetraethylammonium } \\
\text { (TEA) }\end{array}$ & 130.25 \\
\hline
\end{tabular}




\begin{tabular}{|c|c|c|c|}
\hline \multicolumn{4}{|c|}{ Physiological condition buffer for control experiments } \\
\hline Buffer & Concentration & Components & M.W. (g/mole) \\
\hline \multirow[b]{3}{*}{ HEPES Buffer } & $140 \mathrm{mM}$ & $\mathrm{NaCl}$ & 58.44 \\
\hline & $5 \mathrm{mM}$ & $\mathrm{KCl}$ & 74.55 \\
\hline & $2 \mathrm{mM}$ & $\mathrm{CaCl}_{2} \cdot 2 \mathrm{H}_{2} \mathrm{O}$ & 147.02 \\
\hline Plus AP5 and & $1.5 \mathrm{mM}$ & $\mathrm{MgCl}_{2} \cdot 6 \mathrm{H}_{2} \mathrm{O}$ & 203.3 \\
\hline Verapamil & $10 \mathrm{mM}$ & Glucose & 180.16 \\
\hline \multirow[t]{3}{*}{$(\mathrm{pH}=7.4)$} & $25 \mathrm{mM}$ & HEPES & 238.31 \\
\hline & $100 \mu \mathrm{M}$ & AP5 & 197.13 \\
\hline & $30 \mu \mathrm{M}$ & Verapamil & 491.06 \\
\hline
\end{tabular}

\begin{tabular}{|c|c|c|c|}
\hline \multicolumn{4}{|c|}{ LTP induction buffer plus AP5 and Verapamil for control experiment } \\
\hline Buffer & Concentration & Components & M.W. (g/mole) \\
\hline \multirow{9}{*}{$\begin{array}{c}\text { LTP } \\
\text { Inhibition } \\
\text { Buffer } \\
(\mathrm{pH}=7.4)\end{array}$} & $140 \mathrm{mM}$ & $\mathrm{NaCl}$ & 58.44 \\
\hline & $5 \mathrm{mM}$ & $\mathrm{KCl}$ & 74.55 \\
\hline & $5 \mathrm{mM}$ & $\mathrm{CaCl}_{2} \cdot 2 \mathrm{H}_{2} \mathrm{O}$ & 147.02 \\
\hline & $0.1 \mathrm{mM}$ & $\mathrm{MgCl}_{2} \cdot 6 \mathrm{H}_{2} \mathrm{O}$ & 203.3 \\
\hline & $10 \mathrm{mM}$ & Glucose & 180.16 \\
\hline & $25 \mathrm{mM}$ & HEPES & 238.31 \\
\hline & $25 \mathrm{mM}$ & $\begin{array}{l}\text { Tetraethylammonium } \\
\text { (TEA) }\end{array}$ & 130.25 \\
\hline & $100 \mu \mathrm{M}$ & AP5 & 197.13 \\
\hline & $30 \mu \mathrm{M}$ & Verapamil & 491.06 \\
\hline
\end{tabular}




\begin{tabular}{|c|r|l|r|}
\hline \multicolumn{3}{|c|}{ HEPES buffer plus Lat-A for control experiment } \\
\hline \multirow{4}{*}{ Buffer } & Concentration & \multicolumn{1}{|c|}{ Components } & M.W. (g/mole) \\
\hline \multirow{4}{*}{$\begin{array}{c}\text { Actin } \\
\text { depolymerization } \\
\text { buffer } \\
\text { (pH=7.4) }\end{array}$} & $140 \mathrm{mM}$ & $\mathrm{NaCl}$ & 58.44 \\
\cline { 2 - 4 } & $5 \mathrm{mM}$ & $\mathrm{KCl}$ & 147.02 \\
\cline { 2 - 4 } & $2 \mathrm{mM}$ & $\mathrm{CaCl}_{2} \cdot 2 \mathrm{H}_{2} \mathrm{O}$ & 203.3 \\
\cline { 2 - 4 } & $1.5 \mathrm{mM}$ & $\mathrm{MgCl}_{2} \cdot 6 \mathrm{H}_{2} \mathrm{O}$ & 180.16 \\
\cline { 2 - 4 } & $10 \mathrm{mM}$ & $\mathrm{Glucose}$ & 238.31 \\
\cline { 2 - 4 } & $25 \mathrm{mM}$ & $\mathrm{HEPES}$ & 421.55 \\
\hline
\end{tabular}




\subsubsection{Living cell chamber system}

Molecular dynamics within living cells are very sensitive to the changes of temperature; therefore it is very important to keep the temperature constant while performing experiments. To keep the constant temperature, a flow chamber with buffer circulation is necessary. Before the actual experiments, neuron cells were selected and cut from the culture membrane inserts, and then fixed it tightly between coverslip and circulation chamber for observations. A bathing and recording chamber (Harvard Apparatus) was connected to two solution pumps (Harvard Apparatus) that can simultaneously pump into and suck off buffer solution. A dual channel bipolar temperature controller (CL-20, Harvard Apparatus) was connected to the sample holder to control the temperature at a constant level. Buffer solution was not circulated during FCS data acquisition to avoid stage vibration that will result in erroneous autocorrelation curves.

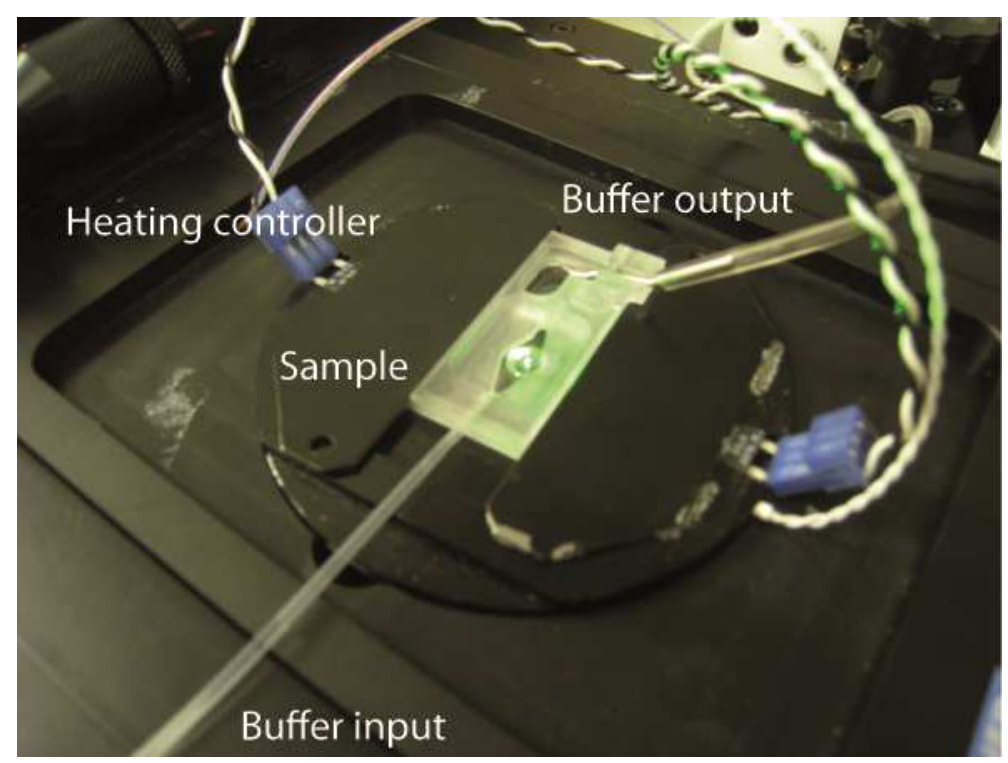

Figure 2.1.1 Living cell chamber system. Neuron samples were kept in a constant temperature $\left(\sim 35^{\circ} \mathrm{C}\right)$ by a heating controller. During FCS measurements, the buffer circulation was stopped to prevent stage vibration. 


\subsection{Methods}

\subsection{1 fCherry and eGFP-actin transfection}

In order to visualize the morphological changes of dendritic spines, neurons were transfected with expression vectors carrying a farnesylated form of mcherry (fcherry) (Shaner et al, 2004). Another transfection was done by using expression vectors that carry eGFP-actin (CA Schoenenberger, University of Basel, Switzerland) for the observation of actin dynamics by using 2P-FCS. Both transfections were under the control of a cytomegalovirus (CMV) promoter. Co-expression of eGFP-actin and fcherry was done by our collaborators from TUBraunschweig and further confirmed by fluorescence microscopy.

\section{(1) Particle-mediated gene transfer:}

By means of gold particle mediated gene transfer, the Helios Gene Gun System (BioRad) was applied to transfect hippocampal slice cultures (pyramidal cells in the CA3 region) after 14-18 days in vitro (DIV). For the co-transfection via gene gun, eGFP-actin and fcherry were mixed with a ratio of 1:2 for a total amount of $2 \mathrm{~g}$ DNA/mg gold particles. The slices were transfected by shooting at a pressure of 80-100 psi, and a filter with a pore size of $3 \mu \mathrm{m}$ was used to prevent gold clumps from damaging the slices. 2P-FCS measurements of actin dynamics were performed 12-24 hours after the transfection.

\section{(2) Single cell electroporation:}

The single cell electroporation was performed by using the Axoporator 800A (Axon Instruments/Molecular Devices Corp.) within pyramidal cells in the CA3 region of hippocampal slice cultures after 14-18 DIV. A 1:1 DNA ratio eGFP-actin to fcherry was used with a total DNA concentration of $100 \mathrm{ng} / \mu 1$ for the co-electroporation. The slices were kept in sterile 1x Hank's Buffered Salt Solution (HBSS) and electroporated with a train of stimuli. The process of electroporation was performed by $200 \mathrm{~Hz}$ stimulation for $100 \mathrm{~ms}$ with each stimuli $1 \mathrm{~ms}$ duration at $5 \mathrm{~V}$ amplitude. The tip resistance of the electrode $(1.5 / 0,86 \mathrm{~mm}$; GC150F-10, Harvard Apparatus) was controlled by a pressure gauge (GDH200, Greisinger) with resistance of 5-7 M $\Omega$ at a pressure of 10-20 mbar. The slices were used for FRAP measurements ${ }^{*} 24 \mathrm{~h}$ after single cell electroporation.

\footnotetext{
${ }^{*}$ Experiments were done by Y.K.
} 


\subsubsection{Living cell measurements}

\section{(1) Image recording and FCS measurement:}

For the purpose of monitoring the morphological modulations of dendritic spines during cLTP, the method of maximum projection images were often used to characterize the quantity of size changes. Firstly, the neuron images were collected by a conventional fluorescence microscopy (FV1000, Olympus), which is equipped with a 60x water immersion objective lens and a CCD camera of $1024 \times 512$ pixels, with an interval of 10 minutes. The $\mathrm{Z}$ sectioning was done by scanning $\mathrm{z}$-axis with a step of $0.5 \mu \mathrm{m}$.

Before cLTP, the hippocampal slice cultures were kept within warm HEPES buffer solution at $35^{\circ} \mathrm{C}$. The induction of cLTP was performed by replacing the HEPES buffer solution with TEA contained stimulation solution. After 10 minutes bathing for stimulation, the TEA contained solution was then washed out by HEPES buffer solution. Detailed buffer recipes are shown in Table 2.1.1.

For the 2P-FCS measurements, the two-photon spot was placed right on the center of dendritic spines before and after cLTP stimulation. Every measurement was 10-15 seconds for 6 rounds to get nice autocorrelation curves. With spine enlargement after 30 minute of cLTP induction, another FCS measurement was performed by the same procedure. Variations of spine shape (Fischer et al, 1998) or sample dislocation could be problems for observing actin dynamics within dendritic spine by FCS. However, the time scales of shape variation or sample dislocation are much larger than that for actin dynamics, which could result in significantly slow diffusion mobility.

\section{(2) Fluorescence Recovery After Photobleaching (FRAP):}

FV1000 with laser wavelength of 488nm was used to excite eGFP molecules in FRAP experiment". Single spines of secondary or tertiary dendritic branches from CA3 hippocampal neurons between 15-19 DIV were used for the measurements. The low level $(\sim 6.9 \mu \mathrm{W})$ excitation laser power was applied to gain a reasonable signal-to-noise ratio for the observation and at the same time, to reduce the photobleaching as much as possible. The scan speed was set to $8 \mu$ s per pixel for a total image size of $640 \times 128$ pixels. The photobleaching of a single spine was performed by using the commercial FRAP unit (FV5-LDPSU, Olympus)

\footnotetext{
${ }^{*}$ Experiments were done by Y.K.
} 
at a wavelength of $405 \mathrm{~nm}$ with a power up to $\sim 2.3 \mathrm{~mW}$ for $25 \mathrm{~ms}$. To determine the baseline and the fluorescence recovery, several images were taken at intervals of 2 seconds before and after the photobleaching.

\subsubsection{Data analysis}

\section{(1) $\underline{\text { FRAP }}$}

The region of interest (ROI) around the bleached spines was defined before FRAP* analysis via Olympus software FV1000. Of course, the background fluorescence was subtracted before calculating the averaging intensity from the ROIs. A bleaching correction derived from neighboring regions was also included. Fluorescence intensity from each spine was then normalized to the pre-bleach levels. The statistical analysis was performed by using the Microsoft Excel or the GraphPad Prism. The FRAP* experimental data was tested by applying an unpaired two-tailed Student t-test point by point. Values of $\mathrm{p}<0.05$ were considered as significant. All data are presented as mean \pm standard error of the mean (s.e.m.).

\section{(2) 2P-FCS}

Actin dynamics within dendritic spines was measured by 2P-FCS before and after TEA stimulation, and the images of morphological changes due to cLTP were further analyzed using the Fiji image process open software. The statistical analysis was performed by using the Microsoft Excel or the GraphPad Prism. The statistic data from MCF-7 cells were tested by applying an unpaired two-tailed t-test. To compare actin dynamics in the same dendritic spine before and after TEA induced cLTP, the statistics were done by applying the paired t-test in single spines. Values of $\mathrm{p}<0.05$ were considered as significant. All data are presented as mean \pm standard error of the mean (s.e.m.).

\footnotetext{
${ }^{*}$ Analysis was done by Y.K.
} 


\subsubsection{Two photon fluorescence correlation spectroscopy (2P-FCS) and Wide-field fluorescence microscopy (FM)}

A home-built setup which combines two photon fluorescence correlation spectroscopy (2P-FCS) and conventional wide field fluorescence microscopy (FM) was used for this study. A Chameleon titanium-sapphire laser (Coherent, USA) was used as a light source for 2P-FCS measurements. Excitation with $87 \mathrm{MHz}$ repetition rates, $900 \mathrm{~nm}$ in wavelength, and $12 \mathrm{~mW}$ of power in the back aperture of objective lens was used to excite eGFP-actin. A continuous wave laser with wavelength at 568nm was used for observing morphology of fCherry labeled dendritic spine head.

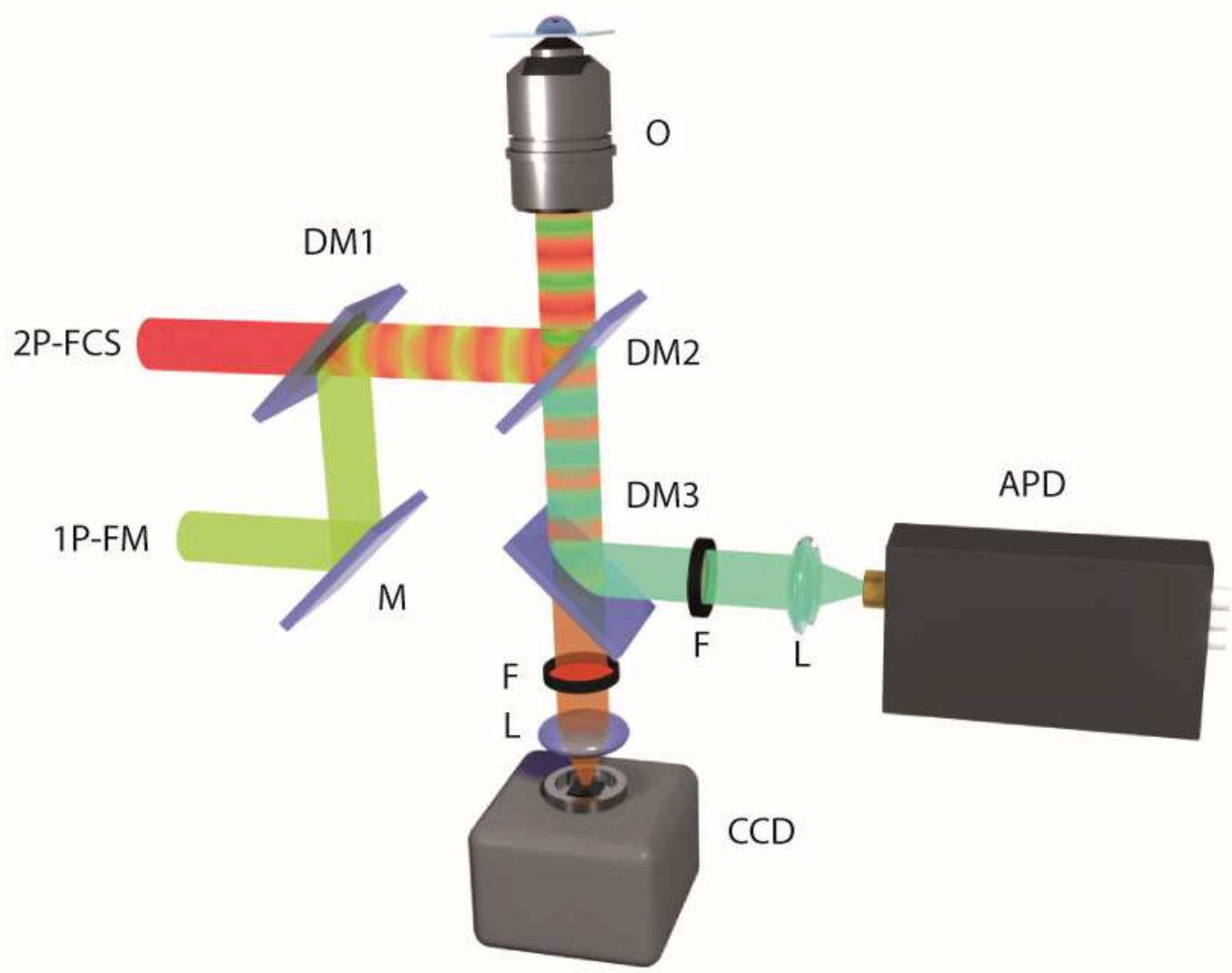

Figure 2.2.1 Home-built 2P-FCS combined with FM. A pulsed Ti:Sa laser and continuous wave one-photon laser were used as light sources. Excitation laser lines were reflected by a special designed dichroic mirror (DM2). DM2 also permits the transmission of emission light. Filters (F) are fixed in front of detectors. CCD is used for image recording and APD is used to record fluorescence fluctuation for FCS calculation. (DM: dichroic mirror, F: filter, L: lens, O: objective lens, M: mirror, APD: avalanche photodiodes, CCD: charged couple device) 
As shown in Figure 2.2.1, two excitation laser lines were coupled to an inverted microscope (IX71, Olympus) and reflected to the back aperture of an objective lens (UPLSAPO 60x, NA=1.2, water immersion, Olympus) through a customized dichroic mirror, DM2, (AHF, Germany). The transmission property of this dichroic mirror is shown in Figure 2.2.2. It reflects both laser light sources at $568 \mathrm{~nm}$ and $900 \mathrm{~nm}$ and permits the transmission of emission light from fluorescence molecules (eGFP and fCherry).

A two-photon rejection filter, E700SP2 (AHF, Germany), was used to block the scatter lights from 2P excitation light source (not shown in Figure 2.2.2). The emission light was further divided into two parts by DM3 (590 DCXR, AHF, Germany) that can further be recorded by corresponding detectors. Before being recorded by CCD and APD detectors (CCD: F-view II, Olympus, APD: SPCM-AQR-13, Perkin-Elmer), emission signals were filtered by appropriate band-pass filters (HQ 645/75 for fCherry and HQ 535/50 for eGFP). Another lens was used in the front of the APD detector to focus emission signals in a small chip area. Signals gathered from APD were further analyzed by a time correlated single photon counting card (TimeHarp 200, PicoQuant $\mathrm{GmbH}$ ). Correlation curves were fitted by using a home-built program (provided by Matthias Grunwald).

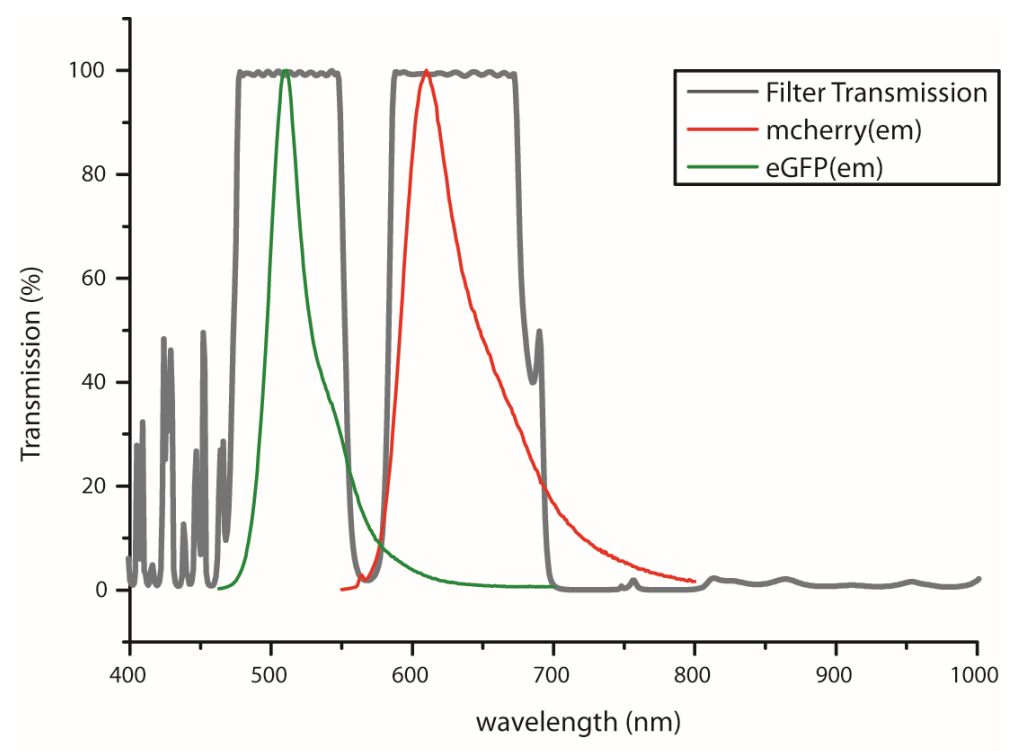

Figure 2.2.2 Special designed dichroic mirror (DM2). Characteristics of the dichroic mirror designed for the simultaneously observation of two different labeling, fCherry and eGFP. (fCherry: Ex:568nm, Em(max):610nm; eGFP: Ex:900nm, Em(max): 507nm) 


\subsubsection{Co-localization of focal plane between 2P-FCS and FM}

The co-localization adjustment of both focal planes from one-photon and two-photon excitation is important to ensure accurate experimental observation. By using the double transfected neuron sample, fCherry for OPE and eGFP-actin for TPE, the co-localization in the XYZ dimensions can be confirmed by simultaneously recording the sharpest images and the maximum eGFP emission. In Figure 2.2.3, with in-focus image in the XY plane observed by OPE fluorescence microscopy, the emission intensity of eGFP is higher while hitting the dendritic spine by two-photon spot, as highlighted with red square area. On the other hand, a relative dimmer emission signal is seen while only hitting the background area without dendritic spine (yellow square area).

Next, the objective lens was adjusted along the Z-axis to record the changes in fluorescence intensity emitted from focal plane. As demonstrated in Figure 2.2.4, the maximum emission from TPE can be observed only in the position $\mathbf{A}$ that the in-focus image was seen by fluorescence microscopy. The fluorescence beads and neuron samples were used for testing. The emission intensities from TPE excitation in position $\mathbf{A}$ are as follows: fluorescence beads: $3 \times 10^{5}$ (a.u.) and neuron cells: $2 \times 10^{4}$ (a.u.). Compared with that in position $\mathbf{B}$ and $\mathbf{C}$, one can find smaller emission intensity (fluorescence beads: $2 \times 10^{4}$ (a.u.); neuron cells: $4.7 \times 10^{3}$ (a.u.)).

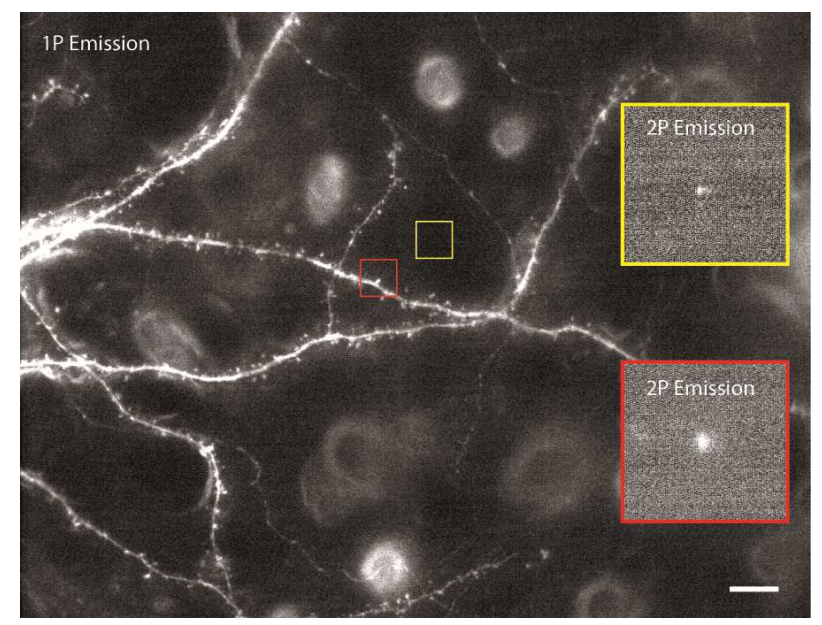

Figure 2.2.3 Co-localization between 1P and 2P excitation in X-Y plane. The red square shows the hitting of two-photon excitation onto the dendritic spine and therefore a brighter emission signal from eGFP-actin molecules. The yellow square shows only a background signal without hitting dendritic spine. (Scale bar: $10 \mu \mathrm{m}$ ) 

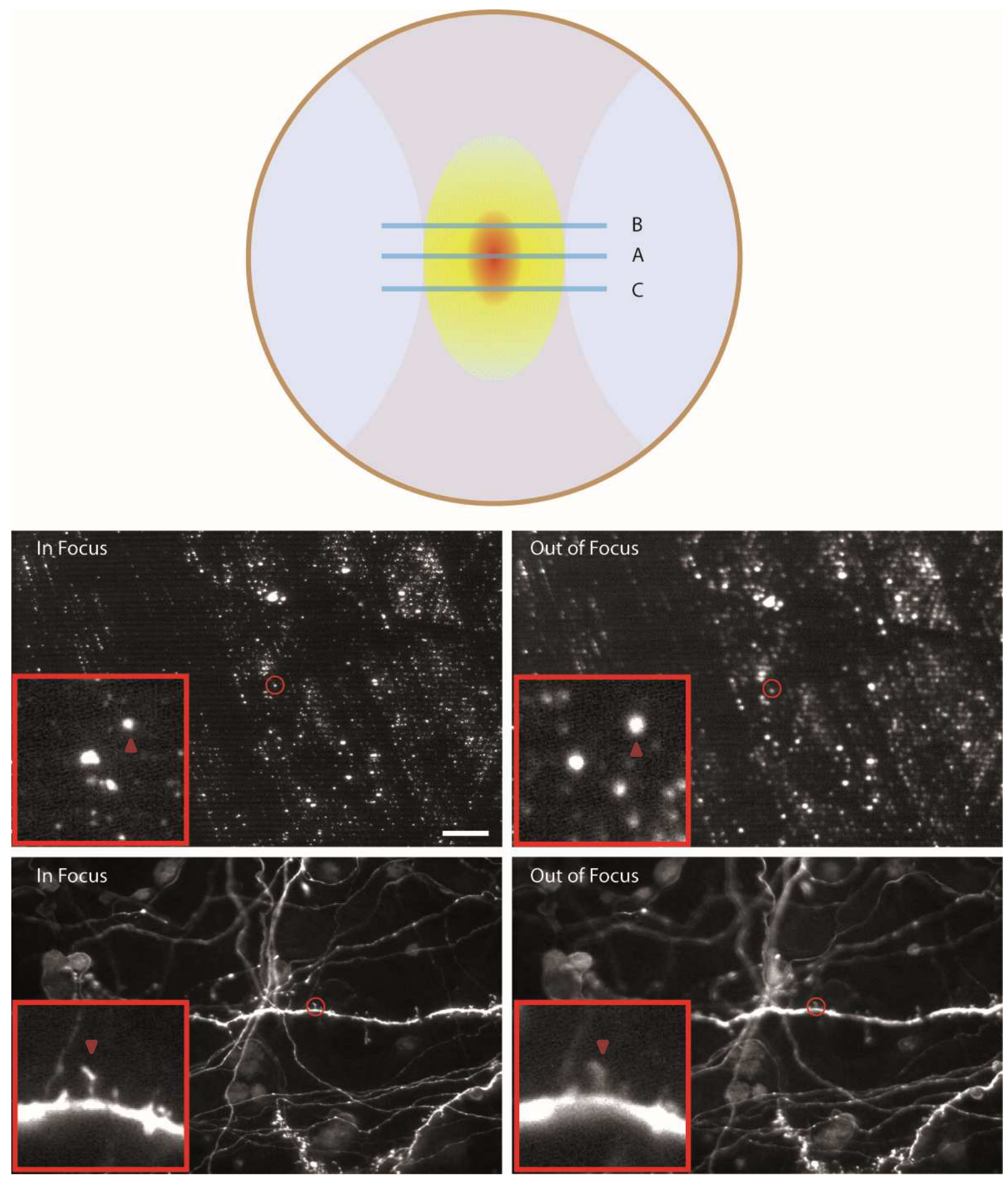

Figure 2.2.4 Co-localization between OPE and TPE in $Z$ plane. The intensity recorded from $2 \mathrm{P}$ emission in APD depending on the focus positions. The emission intensity is at its maximum with the excitation light source in position $\mathbf{A}$ (in-focus). The counts were relatively smaller if images are out of focus. Fluorescence beads and fixed neuron cells were used for testing. (Scale bar: $10 \mu \mathrm{m}$ ) 


\section{Results and Discussions}

\subsection{Free diffusion of GFP measured by 2P-FCS}

Green fluorescence protein (GFP) contains 238 amino acids ( $27 \mathrm{kDa}$ ) (Chalfie et al, 1994; Tsien, 1998). It emits bright fluorescence when excited by OPE at $~ 488 \mathrm{~nm}$ or by TPE at $~ 900 \mathrm{~nm}$. GFP is the most common label for visualizing molecular dynamics within living cells, owing to its high photo-stability and the possibility to express in living cell without interfering the normal functions of fused target proteins. Before performing FCS experiments in GFP expressing living cells, some important parameters, e.g. proper excitation intensity or focal volume, are crucial for the 2P-FCS observation.

Finding the optimal excitation intensity is important because higher intensities lead to faster photobleaching of fluorescent molecules, which in turn distorts FCS data. Photobleaching can be inferred by several parameters in FCS measurements, such as the shortened diffusion time and the increase of autocorrelation amplitude (Kim et al, 2005; Petrasek \& Schwille, 2008).

According to previous studies, the optimal TPE wavelength for exciting GFP molecules is $\sim 900 \mathrm{~nm}$ (Kim et al, 2005). Subsequently, FCS measurements of Annexin VeGFP $(\sim 35 \mathrm{kDa})$ in solution were performed at $900 \mathrm{~nm}$ with various excitation intensities. The total count rate and particle brightness were increased in a power-dependent (quadraticdependent) manner up to $30 \mathrm{~mW}$ (Figure 3.1.1 (A) and (B)). The autocorrelation amplitude $\mathrm{G}(0)$ and diffusion time measured at the respective excitation intensities are shown in Fig. 3.1.1 (C) and (D). The decrease of both parameters beyond $30 \mathrm{~mW}$ clearly demonstrates that photobleaching becomes prominent which may result in data misinterpretation.

For living cell experiments, there should be a trade-off between the overall signal intensity and photobleaching effect. In conclusion, based on the power dependent experiments, the best excitation power for eGFP is $12-25 \mathrm{~mW}$. The diffusion time of Annexin V-eGFP measured with $900 \mathrm{~nm}, 12 \mathrm{~mW}$ is used to calculate the diffusion coefficient. With an averaged diffusion time of $0.26 \mathrm{~ms}$, the diffusion coefficient of Annexin V-eGFP in solution at room temperature is determined to be $77 \mu \mathrm{m}^{2} / \mathrm{s}$, which is in line with published data (Dayel et al, 1999; Partikian et al, 1998; Stasevich et al, 2010). 

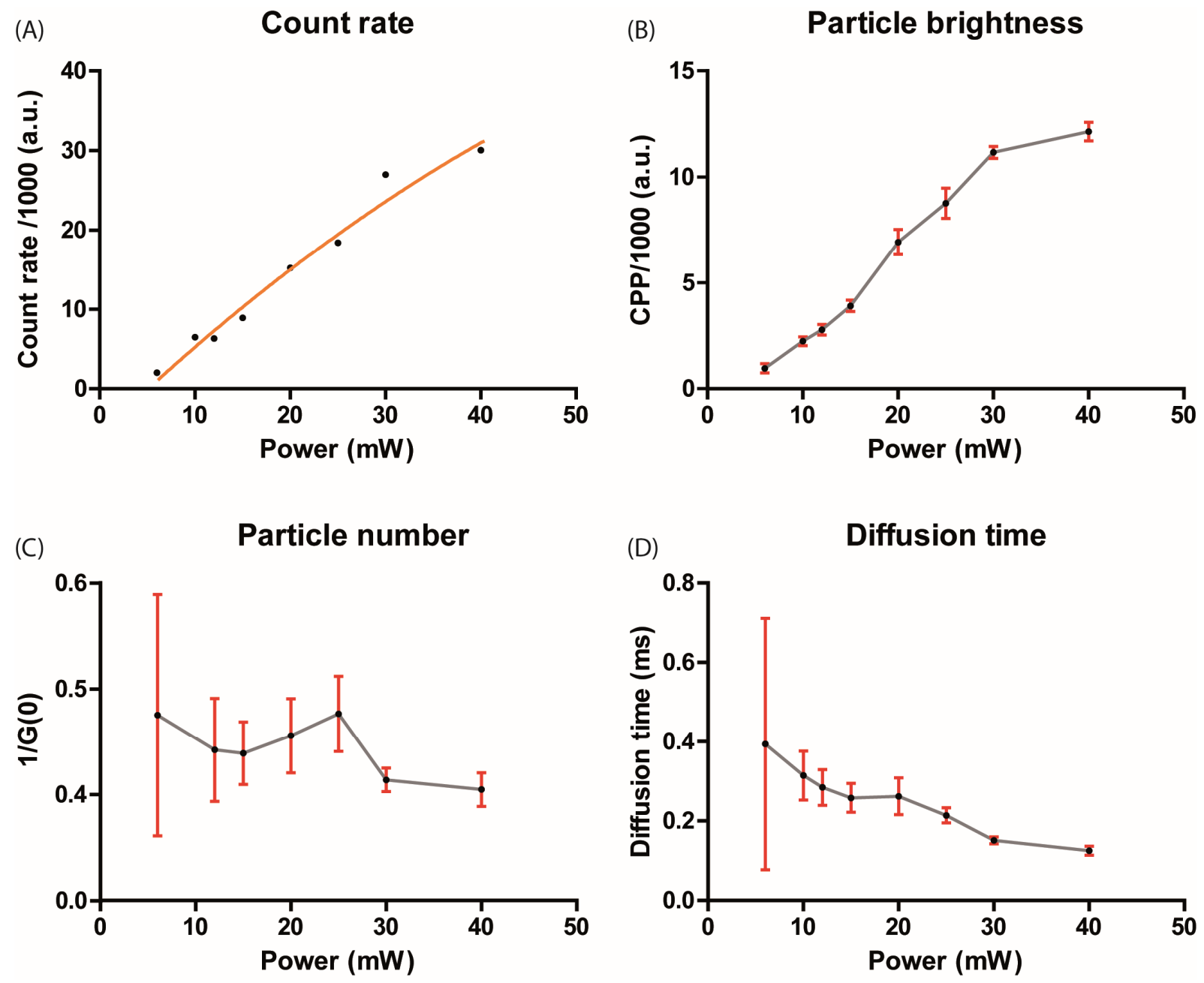

Figure 3.1.1 Parameters of Annexin V-eGFP obtained from power-dependent FCS measurements. (A) The total count rate, (B) particle brightness, (C) particle number and (D) diffusion time. 


\subsection{Autocorrelation curve fitting models}

Autocorrelation curves generated after FCS measurements have to be further fitted with appropriate mathematical models. Based on the inherent molecular diffusion mobility, one can choose models from normal three-dimensional diffusion, anomalous diffusion or active transport modes. As mentioned before, actin structure is always in a dynamic equilibrium to maintain cellular functions, thus, it is important to choose a physical model that best describes this highly heterogeneous dynamic behavior within neurons. The discussions of how to interpret data from autocorrelation curves will be presented in the following content.

Figure 3.2.1 represents schematically the difference between homogeneous and heterogeneous molecular distribution. Molecules with a uniform structure within FCS focal volume result in a more homogeneous intensity fluctuation, as shown in Figure 3.2.1(B). On the contrary, there is a heterogeneous intensity fluctuation if molecules assume non-uniform structures. The difference of homogeneous and heterogeneous molecular compositions can be distinguished from distinct autocorrelation curves. A homogeneous molecular diffusion results in a smooth autocorrelation curve that is represented as the black curve in Figure 3.2.1(C), whereas heterogeneous molecular diffusion is represented in the red one. In this section, we will discuss the choices of fitting models based on the properties of actin dynamics and structural compositions. 
(A)

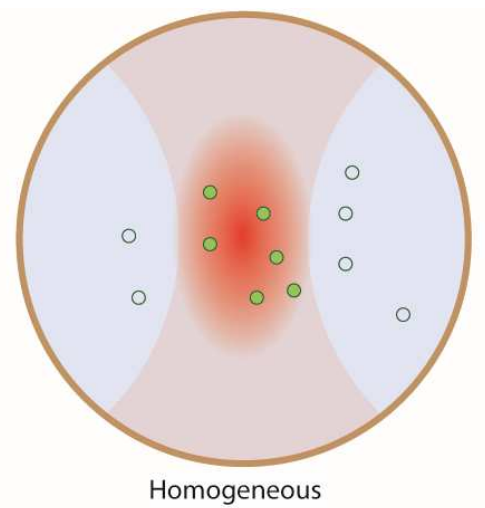

(B)

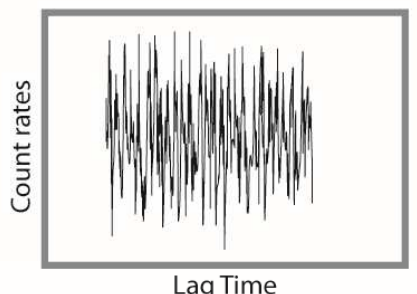

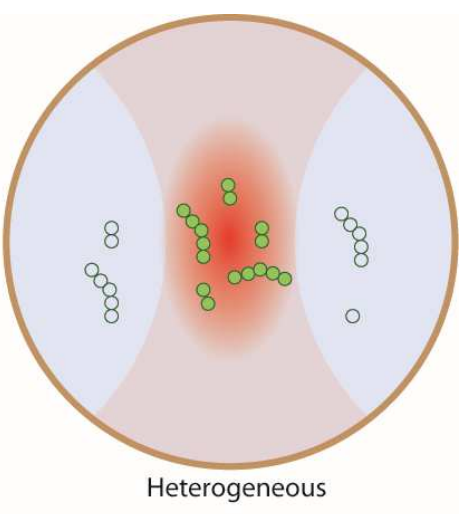

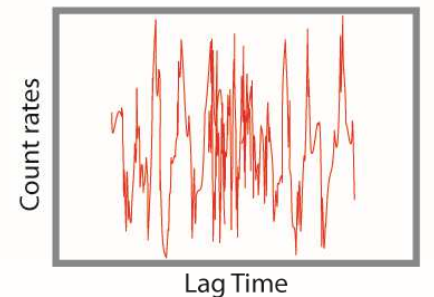

(C)

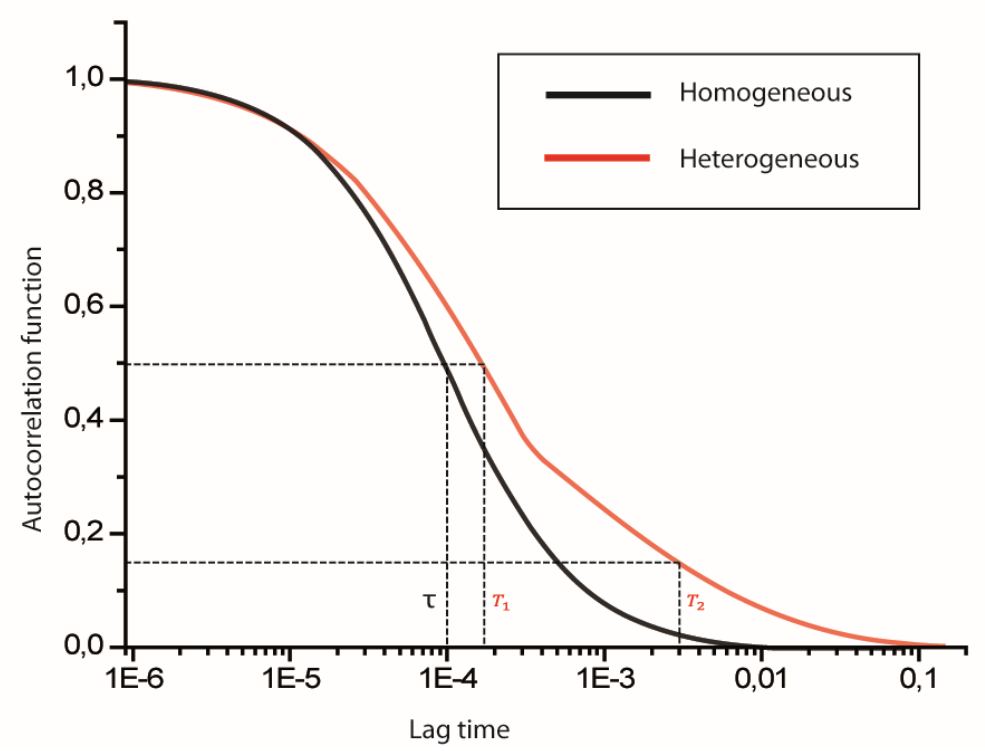

Figure 3.2.1 Homogeneous and heterogeneous diffusion particles. (A) Schematic representation of the difference between homogenous and heterogeneous distribution, (B) Comparisons of intensity fluctuations between homogenous and heterogeneous diffusion (C) The autocorrelation curves illustrate the differences between homogeneous (black curve) and heterogeneous (red curve) populations. 


\subsubsection{Two-component fitting model}

Actin filaments are always in a dynamic equilibrium between actin monomers and filaments. Therefore, we first fit the autocorrelation curve using a two-component model, which is described in equation (3.1) (Lakowicz, 2006; Rhoades et al, 2006; Rusu et al, 2004). Besides two different diffusion times, the corresponding molecular fractions can be derived from this model. Assuming the same particle brightness, relative molecular concentrations can be calculated according to fraction number $\alpha$. Moreover, as shown in Figure 3.2.2, the fraction ratio between two different groups of molecules can be used as an indicator to describe molecular heterogeneity.

The comparison between one- and two-component fittings of the averaged autocorrelation curve (Figure 3.2.3(B)) from several independent FCS measurements (Figure 3.2.3(A)) is shown in Figure 3.2.3(C) and (D). An advantage of averaging the autocorrelation curves is to reduce errors due to photobleaching, which distort the autocorrelation curves at longer correlation time. Compared with one-component fitting model in Figure 3.2.3(C), the smaller residuals of two-component fitting model in Figure 3.2.3(D) denotes better characterization of actin dynamics within living cell. Two different diffusion times derived from this fitting model represent two groups of actin filaments having different degrees of polymerization. In conclusion, several parameters can be derived from the two-component fitting model to describe actin dynamics within living cells: (1) actin filaments with faster diffusion time, (2) actin filaments with slower diffusion time and (3) the molecular fractions of two components.

Based on the two-component fitting method, the very heterogeneous distribution of actin filaments is grouped into two classes. One class consists of shorter forms of F-actin having higher dynamic mobility (diffusion coefficient $\sim 7 \mu \mathrm{m}^{2} / \mathrm{s}$ ). Compared with the diffusion coefficient of freely diffusing eGFP (section 3.1), this dynamic F-actin comprises of $\sim 10$ actin basic units. Another class is F-actin with lower diffusion mobility (diffusion

coefficient $\sim 0.07 \mu \mathrm{m}^{2} / \mathrm{s}$ ), indicating its size is of magnitude about two orders larger than the dynamic one.

$$
G(\tau)=\frac{1}{V_{\text {eff }} \cdot\langle C>} \cdot\left[\left(\frac{\alpha}{1+\frac{\tau}{\tau_{1}}} \cdot \frac{1}{\sqrt{1+\omega^{-2}\left(\frac{\tau}{\tau_{1}}\right)}}\right)+\left(\frac{1-\alpha}{1+\frac{\tau}{\tau_{2}}} \cdot \frac{1}{\sqrt{1+\omega^{-2}\left(\frac{\tau}{\tau_{2}}\right)}}\right)\right]
$$




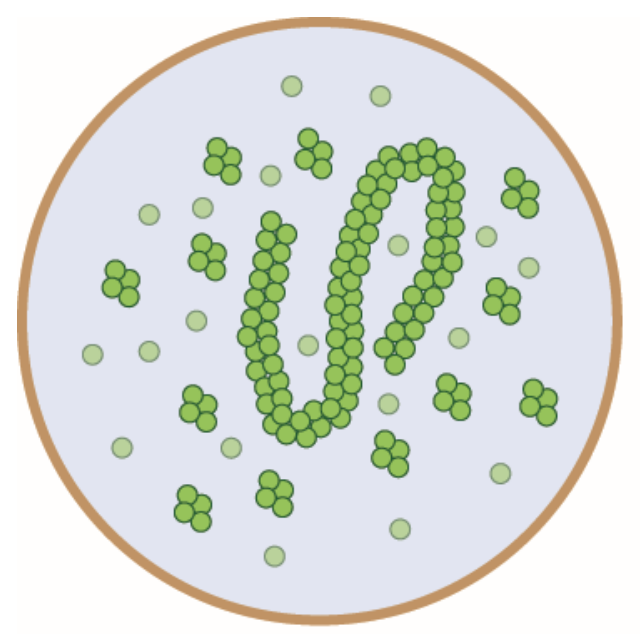

Fraction $=0.91: 0.09$

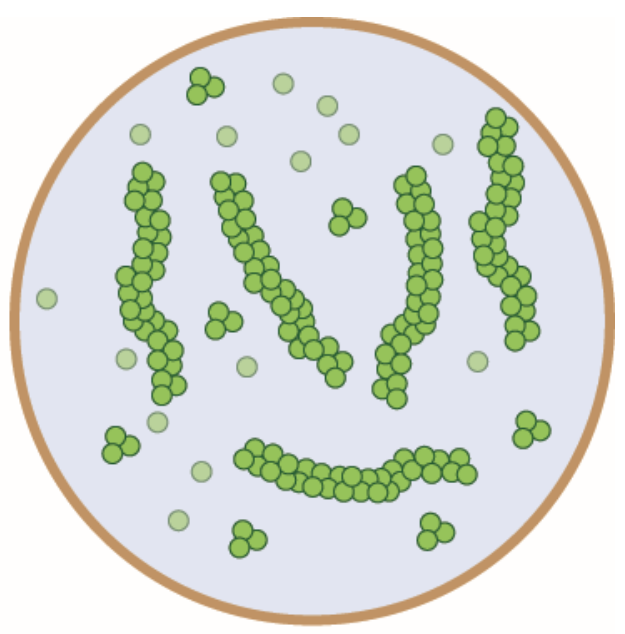

Fraction $=0.58: 0.42$

Figure 3.2.2 Fraction between two distinct compositions within the focal volume. Different degrees of polymerization of fluorescence molecules can be distinguished with FCS measurement using two-component fitting model. The molecular fractions can be used as an indicator to describe molecular heterogeneity. 

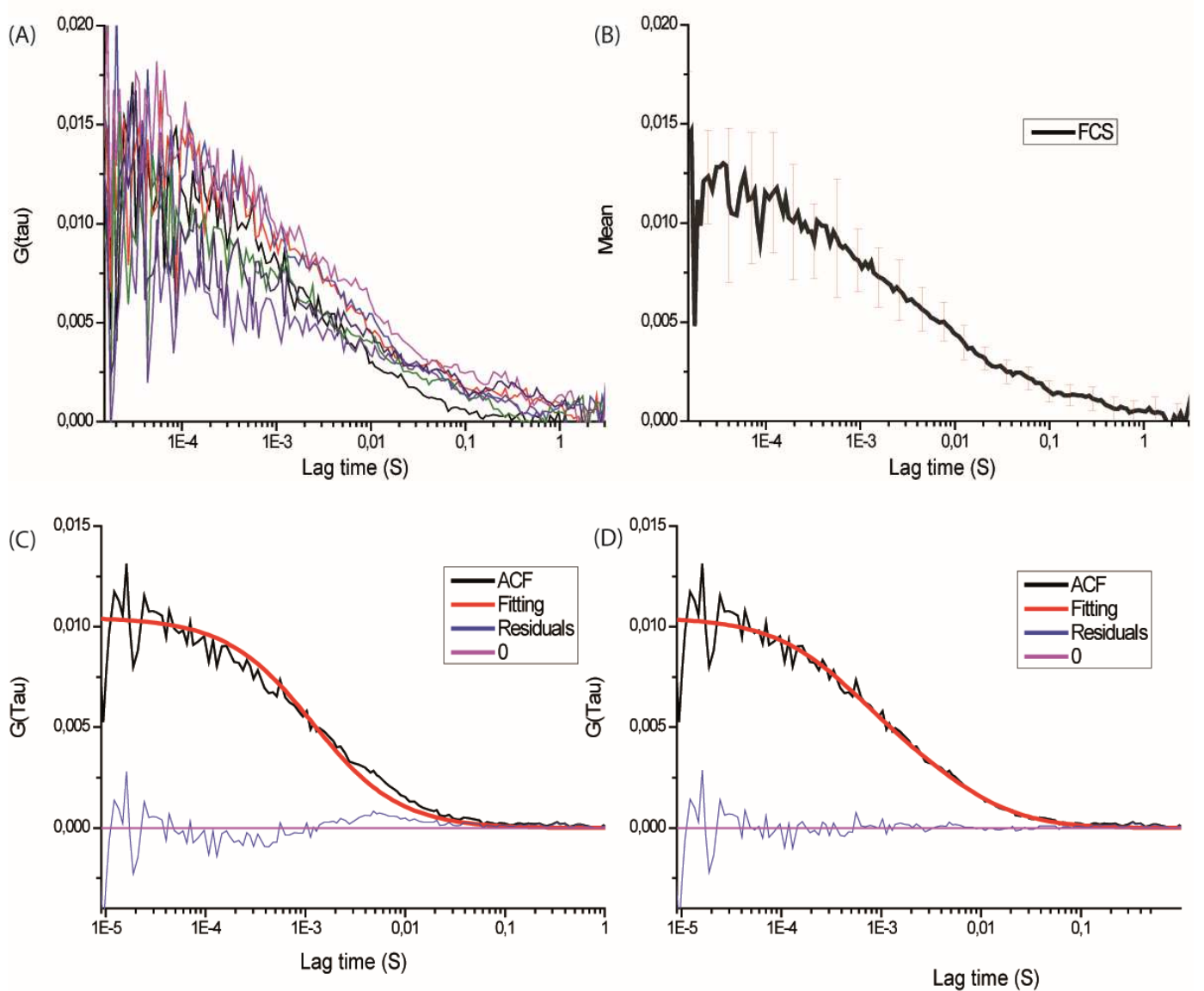

Figure 3.2.3 Statistics and fitting methods to quantify autocorrelation curve of living cells. (A) The autocorrelation curves of actin dynamics measured by 2P-FCS. (B) The averaged autocorrelation curve with error bars. (C) One-component fitting model (red curve), which has a larger residual (blue curve). (D) Two-component fitting model with smaller residuals. 


\subsubsection{Multiple diffusion species with different brightness}

From the two-component fitting model, molecules with different diffusion mobility can be distinguished and the corresponding molecular fractions are also derived to describe the heterogeneity in molecular compositions within the focal volume. However, this fitting model is only applicable when the brightness of the two populations does not differ significantly.

With complicated structural compositions, these two groups of actin filaments with different diffusion mobility represent different degrees of polymerization. Since eGFP was expressed in actin monomers, the different degrees of polymerization in actin filaments result in different brightness. Therefore, there should be significant brightness differences between these two groups. With largely differences in particle brightness, fluorescence fluctuations will be dominated by the brighter molecules. Thus, the absolute particle numbers and brightness is not readily determined. With a highly heterogeneous composition, actin dynamics observed with FCS measurements should be fitted with the brightness taken into account. The formula for fitting the autocorrelation function considering a brightness factor is written as (Lakowicz, 2006; Walla, 2009):

$$
G(\tau)=\frac{I^{2} \cdot N_{1} \cdot M_{1}+(A \cdot I)^{2} \cdot N_{2} \cdot M_{2}}{\left(I \cdot N_{1}+A \cdot I \cdot N_{2}\right)^{2}}
$$

$$
\begin{aligned}
& I=\text { short form intensity (brightness) } \\
& A \cdot I=\text { long form intensity (brightness) } \\
& N_{1}=\text { averaged short form particle number } \\
& N_{2}=\text { averaged long form particle number } \\
& \left.M_{1}=1 /\left(1+\tau / \tau_{1}\right) \cdot 1 /\left(\sqrt{1+\omega^{-2}\left(\tau / \tau_{1}\right)}\right) \text { (mobility term of } N_{1}\right) \\
& M_{2}=1 /\left(1+\tau / \tau_{2}\right) \cdot 1 /\left(\sqrt{1+\omega^{-2}\left(\tau / \tau_{2}\right)}\right)\left(\text { mobility term of } N_{2}\right)
\end{aligned}
$$


According to equation (3.2), the contributions of each different species to the autocorrelation function are proportional to the square of the corresponding particle brightness. Comparing with normal two-component fitting, more parameters can be derived: (1) the brightness of short-form F-actin, (2) the brightness of long-form F-actin, (3) the particle number of short-form F-actin, (4) the particle number of long-form F-actin, and (5) the diffusion time of both groups. 


\subsection{Actin dynamics within living cells}

Based on experimental methods and fitting models mentioned in previous sections, actin dynamics within MCF-7 cells was measured first to characterize the setup. The data shown in Figure 3.3.1 represents the average diffusion time and the corresponding molecular fractions measured at eight different points within the cellular cytosol and fitted with the twocomponent model. A very large heterogeneity in the diffusion time is observed and the actin filaments can roughly be categorized into two groups: short-form F-actin and long-form Factin. The first group is shorter actin filaments with faster diffusion mobility and the second group is longer actin filaments with slower diffusion mobility. The diffusion time of both group are $[0.0023 \pm 0.0002 \mathrm{~s}]$ and $[0.050 \pm 0.006 \mathrm{~s}]$, respectively. Based on the difference in diffusion time, it is estimated that the difference in polymerization degree is a factor of 20.

Although the two-component fitting model cannot precisely describe the absolute particle number and brightness, the relatively molecular fractions can still be compared. The results shown in Figure 3.3.1(B) depict that there are larger amounts of short-form F-actin within the cytosol.

(A)

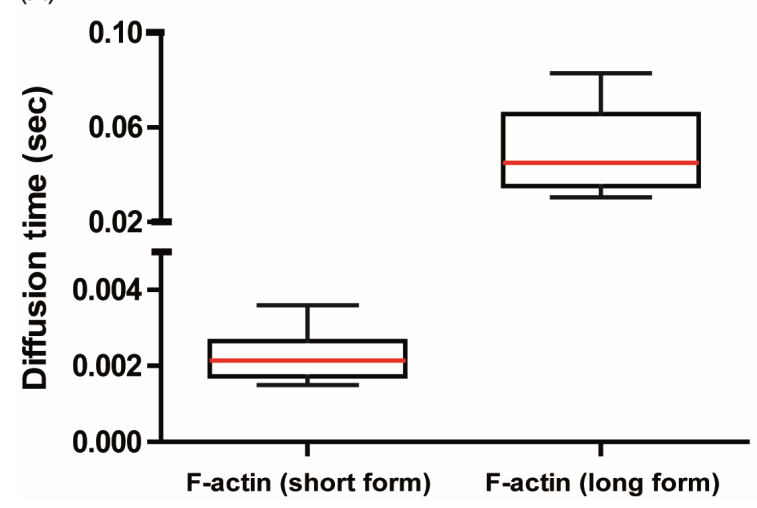

(B)

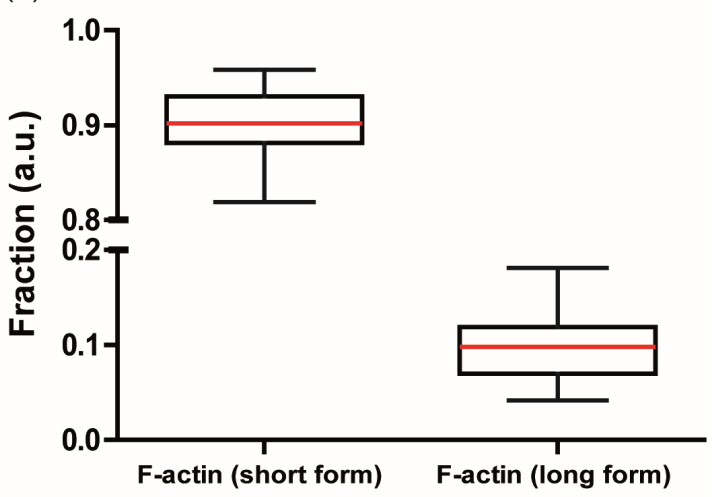

Figure 3.3.1 Actin dynamics within MCF-7 cells. (A) Actin filaments with different dynamics observed by FCS can be classified into two groups: short-form F-actin and longform F-actin. (B) The corresponding molecular fractions of actin filaments. 


\subsubsection{The treatment of Lat-A in MCF-7 cancer cells}

Latrunculin A (Lat-A) is a chemical that is commonly used to sequester actin monomers (Coue et al, 1987; Yarmola et al, 2000), which in turn induce depolymerization of actin filaments. In Figure 3.3.2, MCF-7 cell was treated with Lat-A (4.74 $\mu \mathrm{M})$ to inhibit actin polymerization for FCS measurements and the changes in the parameters derived from autocorrelation curves were compared.

Figure 3.3.2 (A) demonstrates that there is no significant change in diffusion time of the short-form F-actin within the first 30 minutes of incubation, on the other hand, in Figure 3.3.2(B), the disruption of long-form F-actin has already occurred within the first 30 minutes according to its faster dynamics. With longer incubation time up to 60 minutes, the remarkably accelerated diffusion time of short-form F-actin implies that the disruption has also taken place. Comparing the changes in diffusion time of long-form F-actin, the diffusion mobility has increased by a factor of 2 and 4 after 30 and 60 minutes Lat-A treatment.

As expected, actin filaments underwent depolymerization while inducing the disruption of stable actin structure by Lat-A. The observation of decreased diffusion time (increased diffusion mobility) after Lat-A treatment is in line with other studies, which show that actin filaments are cut or disrupted into smaller fragments. The data shown in Figure 3.3.2 (C) and (D) represents the molecular heterogeneity which has increased due to filament disruption after Lat-A treatment. For both actin groups, the molecular heterogeneity has increased significantly within 30 minutes. 
(A)

F-actin(short form)

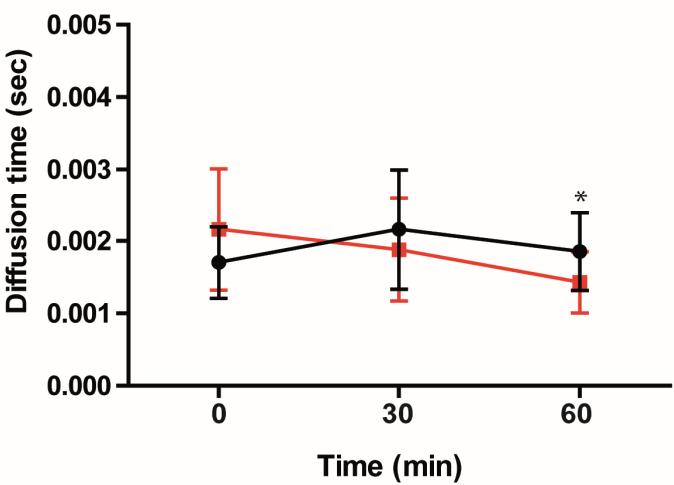

(C)

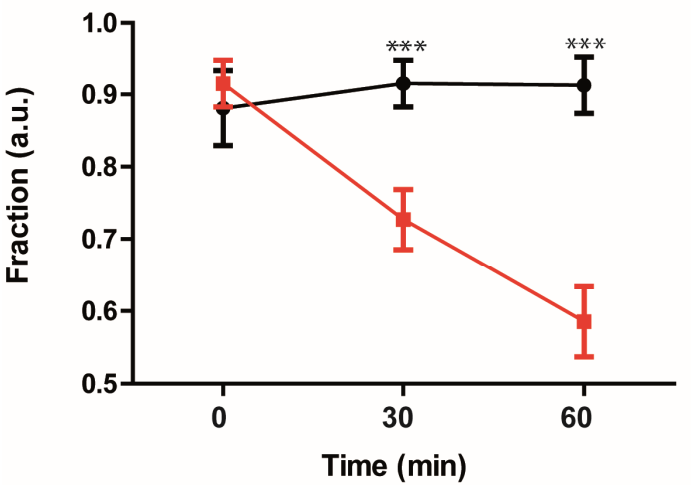

(B)

F-actin(long form)

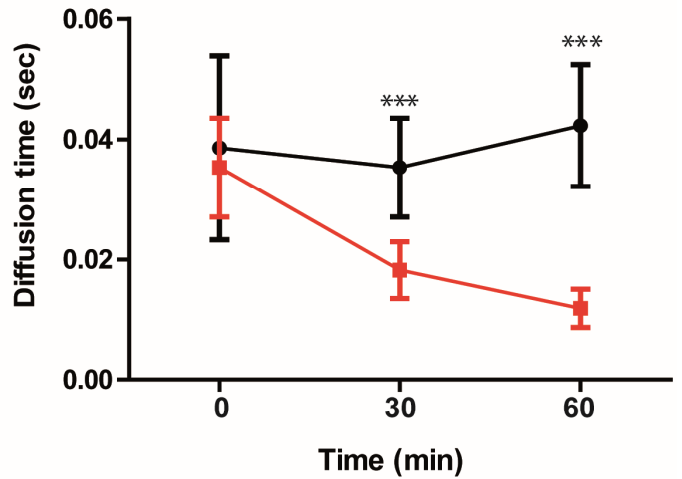

(D)

F-actin(long form)

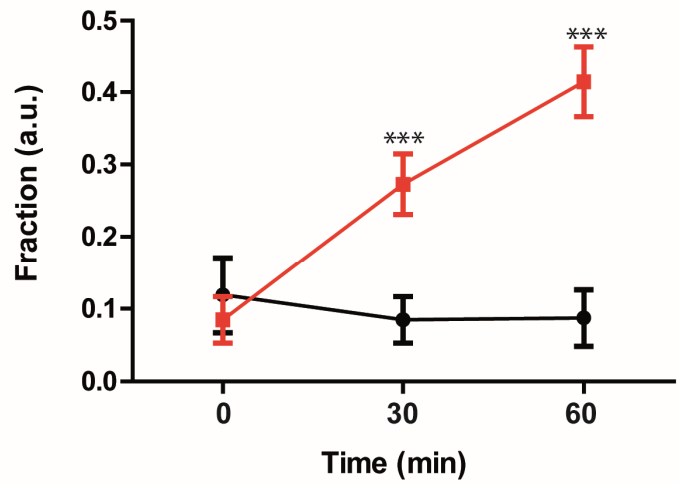

Figure 3.3.2 Actin dynamics within MCF-7 cells treated with Lat-A. With the treatment of Lat-A $(4.7 \mu \mathrm{M})$, actin filaments undergo depolymerization, which disrupts the stable actin structure. The disruption results in faster diffusion dynamics. (A) and (B) show the changes in diffusion time for short-form F-actin and long-form F-actin, respectively. (C) and (D) show the changes in molecular fractions with the function of incubation time. 


\subsubsection{Treatment of Lat-A in dendritic spines}

Lat-A was then applied to induce disruption of actin filaments within dendritic spines. Based on the time scales whereas actin filament within MCF-7 cells underwent disruption, the comparison of actin dynamics measured by FCS was performed at time point 0 (before stimulation) and after 30 minutes treatment (after stimulation).

Figure 3.3.3(A) demonstrates the changes of autocorrelation curves of actin dynamics before and after the neuron had been treated with Lat-A, and the fitting curves before (red) and after (pink) Lat-A treatment were derived from the two-component model with the factor of different particle brightness. Changes in diffusion mobility of actin filaments can be observed from the comparison of normalized autocorrelation curves, as shown in Figure 3.3.3(B), where faster dynamics is exhibited after Lat-A treatment. The fact that the diffusion time decreases for both actin populations after Lat-A treatment was consistent with MCF-7 cells. In addition, the results are in line with earlier studies (Frost et al, 2010; Honkura et al, 2008; Okamoto et al, 2004), which confirmed that the Lat-A treatment induces the breakdown of actin filaments into smaller fragments and therefore a faster mobility is observed.

(A)

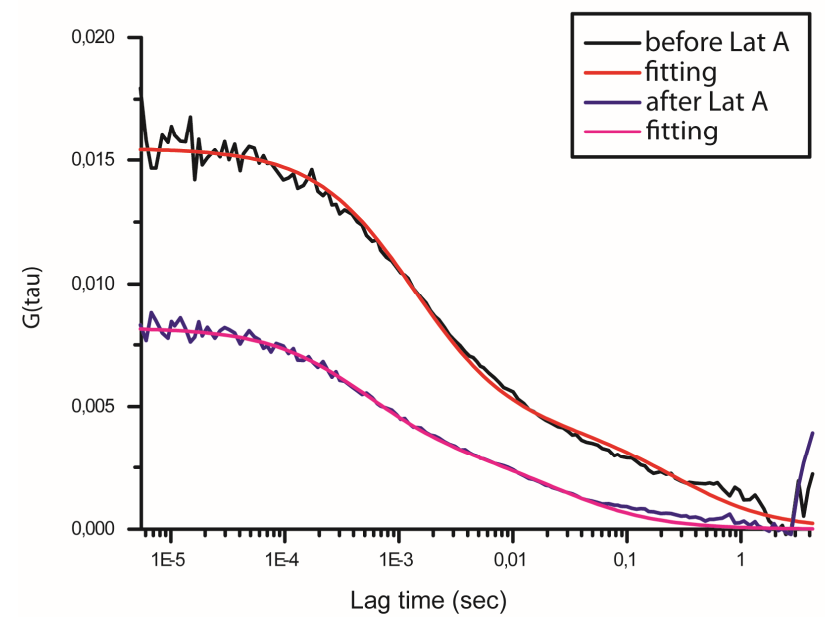

(B)

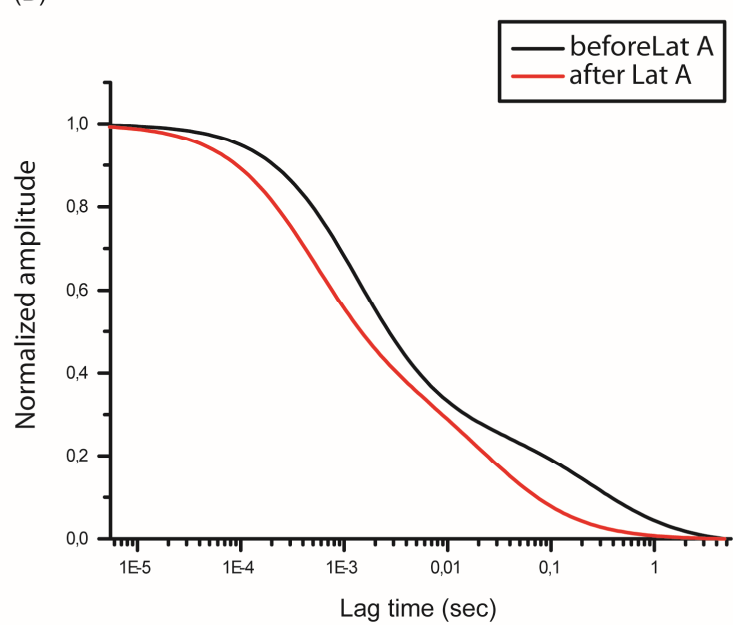

Figure 3.3.3 Actin dynamics within dendritic spines treated with Lat-A. (A) The averaged autocorrelation curves before and after Lat-A treatment (black and blue curves), which were fitted with the different brightness model (red and pink curves). (B) Normalized fitting curves demonstrate the changes in diffusion mobility after Lat-A treatment. 
In Figure 3.3.4, based on two-component fitting, comparisons of molecular diffusion time and fractions are presented to describe actin dynamics before and after Lat-A treatment for 8 single dendritic spines. The decrease of diffusion time in both actin populations and the increase of molecular heterogeneity can be observed after the disruption of longer form actin filaments. However, in order to further scrutinize the changes in actin dynamics and compositions, the factor of different brightness has to be considered while fitting autocorrelation curves.

As can be seen in Figure 3.3.4(A) and (B), the change in diffusion time of short-form F-actin after Lat-A treatment is from $[0.0022 \pm 0.0005 \mathrm{~s}]$ to $[0.0009 \pm 0.0004 \mathrm{~s}]$, and that of long-form F-actin is from $[0.21 \pm 0.04 \mathrm{~s}]$ to $[0.06 \pm 0.01 \mathrm{~s}]$. Furthermore, as represented in Figure 3.3.5 (A) and (B), the particle number of longer form F-actin is increased significantly (310 $\pm 74 \%)$ with the addition of Lat-A. In contrast, one can find that the average particle

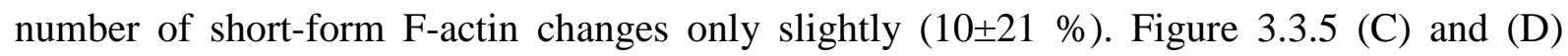
demonstrate that the particle brightness is decreased in both populations of actin filament. For short-form F-actin, it changed from [ $4230 \pm 458$ a.u.] to [2186 \pm 488 a.u.], while it changed from $\left[(352 \pm 31)^{*} 10^{3}\right.$ a.u.] to $\left[(101 \pm 20)^{*} 10^{3}\right.$ a.u.] for long-form F-actin. The changes in diffusion time, particle number and particle brightness are listed in Table 3.1.

Actin filaments become shorter with the treatment of Lat-A based on the results of accelerated diffusion time. The fact that actin filaments have been shortened can be confirmed with the decreased particle brightness. Treatment of neuron with Lat-A has the same effect as for MCF-7 cell. The slight change of particle number in short-form F-actin may likely due to the faster diffusion mobility. However, particle number of longer form F-actin did increase significant after Lat-A treatment due to the disruption of the more stable F-actin filaments. Furthermore, as shown in Figure 3.3.6, the drastic decrease in overall fluorescent signal within the observation focal volume implies the loss of fluorescent molecules. In principle, the concentration of fluorescence molecules is proportional to the overall intensity (Walla, 2009). Therefore, it is clear that the total concentration of actin filaments decreased within dendritic spine after Lat-A treatment. 
(A) F-actin(short form)

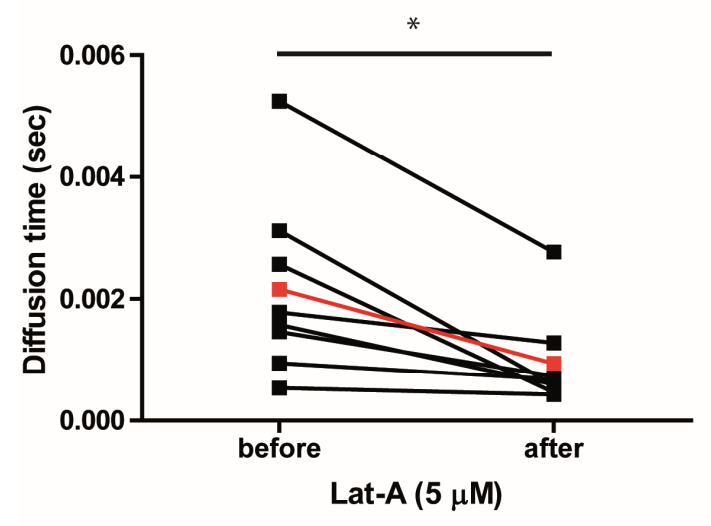

(C)

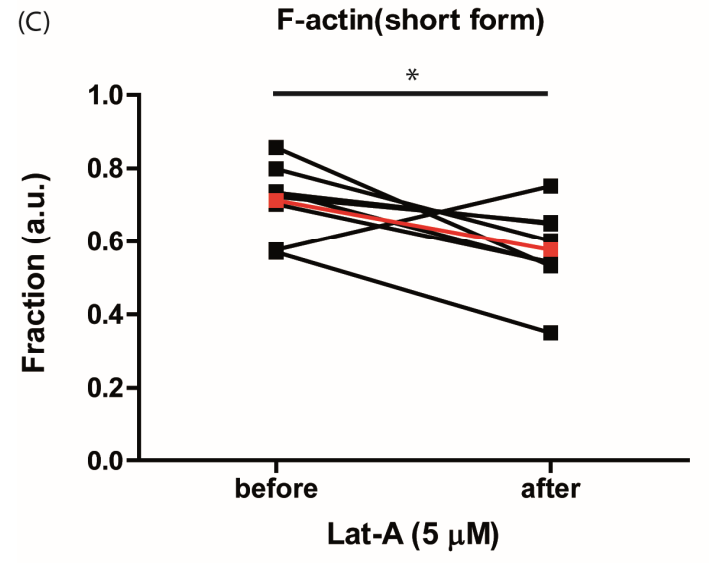

(B) F-actin(long form)

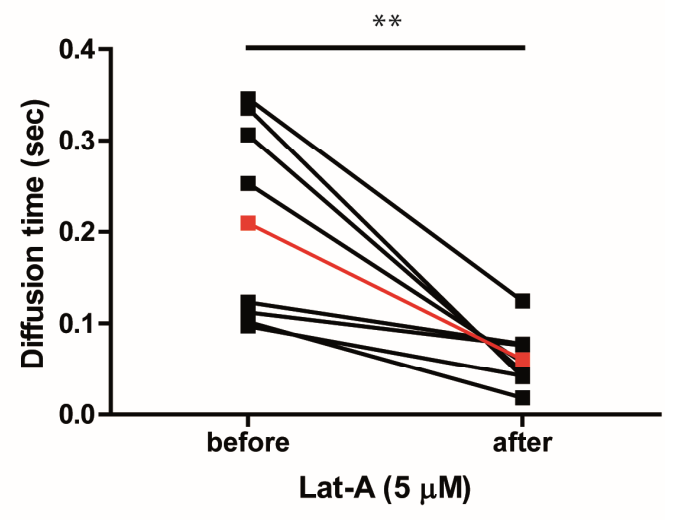

(D)

F-actin(long form)

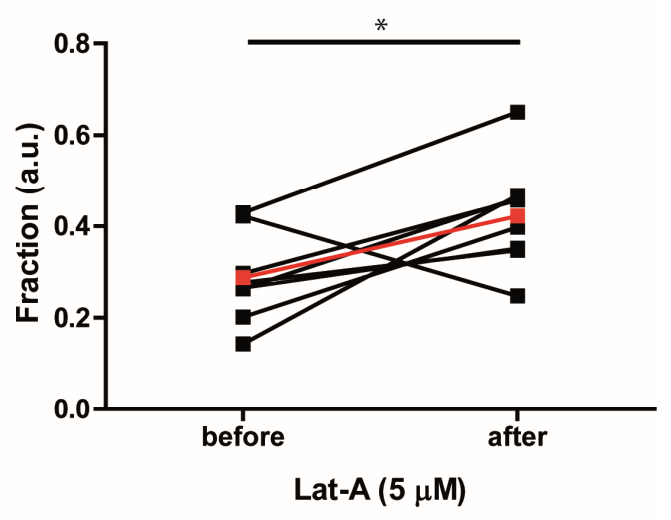

Figure 3.3.4 Changes in actin dynamics and molecular fractions within dendritic spines before and after Lat-A treatment. ( $N=8)$ With the treatment of Lat-A $(5 \mu \mathrm{M})$, actin filaments within dendritic spines undergo depolymerization. This induces the disruption of stable actin structures resulting in faster diffusion of two major populations. (A) and (B) show the decrease of diffusion time in both groups, the short-form and long-form F-actin. ( $\mathrm{p}=0.0111$ and 0.0051 , respectively) (C) The molecular fraction of short-form F-actin decreases with the treatment of Lat-A; (D) The molecular fraction of long-form F-actin increases with the treatment of Lat-A $(\mathrm{p}=0.0367)$ (Average values are shown in red, and the molecular fractions are obtained from two-component fitting). 
(A)

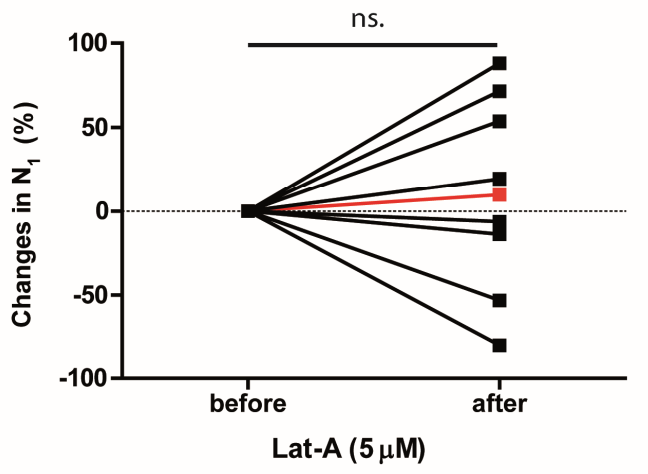

(C)

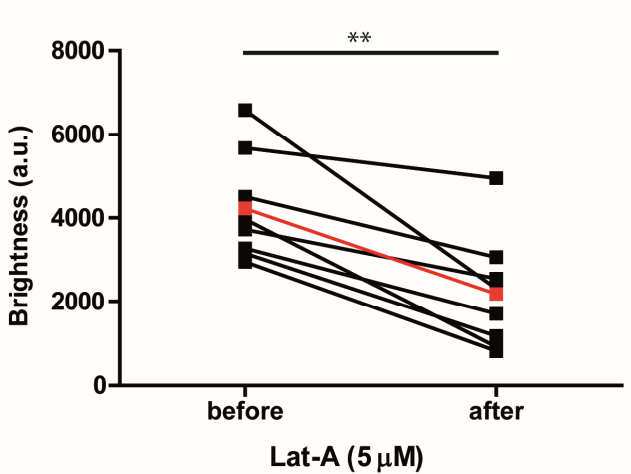

(B)

F-actin(long form)
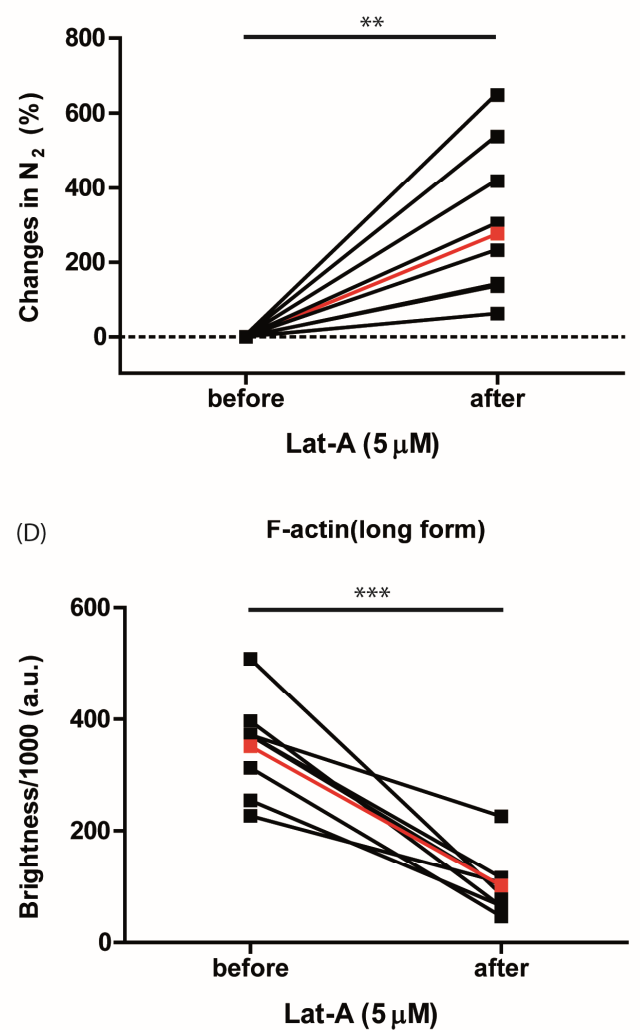

Figure 3.3.5 Changes in particle number and brightness within dendritic spines before and after Lat-A treatment. With the treatment of Lat-A, the actin structure changed due to depolymerization. (A) The changes in averaged particle numbers of short-form F-actin did not alter significantly. (B) The changes in averaged particle numbers of long-form F-actin increased $(\mathrm{p}=0.0040)$. (C) and (D) represent the decrease of particle brightness after Lat-A treatment in both short- and long-form F-actin ( $\mathrm{p}=0.0014$ and 0.0002 , respectively). 


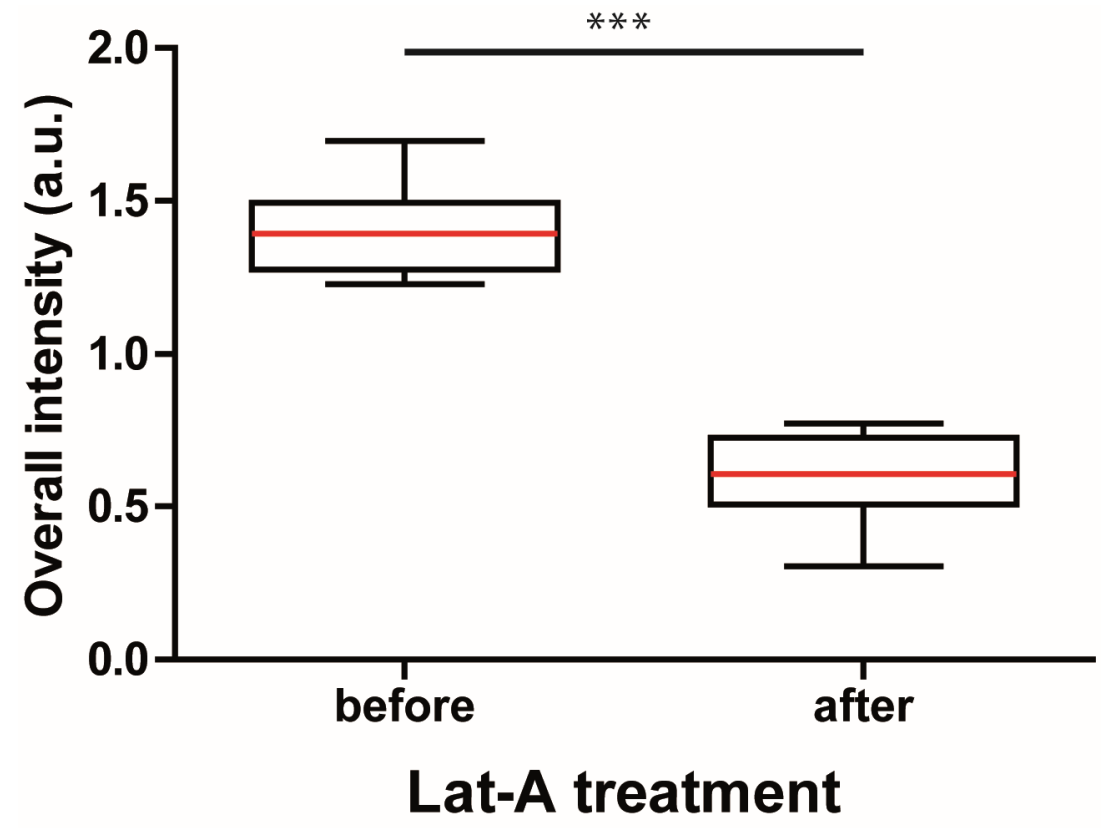

Figure 3.3.6 Changes in overall intensity before and after Lat-A treatments. The significant decrease in overall intensity within the focal volume after Lat-A treatment represents the loss of actin molecules ( $\mathrm{p}=0.0002)$.

Table 3.1 Summary of physical parameters of actin dynamics before and after Lat-A treatment.

\begin{tabular}{|c|c|c|c|}
\hline \multicolumn{2}{|c|}{} & Before Lat-A & After Lat-A \\
\hline \multirow{2}{*}{ Diffusion time (sec) } & Short-form F-actin & $0.0022 \pm 0.0005$ & $0.0009 \pm 0.0004$ \\
\cline { 2 - 4 } & Long-form F-actin & $0.21 \pm 0.04$ & $0.06 \pm 0.01$ \\
\hline \multirow{3}{*}{ Change in particle number (\%) } & Short-form F-actin & 0 & $10 \pm 21$ \\
\cline { 2 - 4 } & Long-form F-actin & 0 & $310 \pm 74$ \\
\hline \multirow{3}{*}{ Particle brightness (a.u.) } & Short-form F-actin & $4230 \pm 458$ & $2186 \pm 488$ \\
\cline { 2 - 4 } & Long-form F-actin & $(352 \pm 31) * 103$ & $(101 \pm 20) * 103$ \\
\hline
\end{tabular}




\subsection{Chemical induction of long-term potentiation (cLTP)}

Long-term potentiation is one of the important processes related to learning and memory (Bliss \& Collingridge, 1993; Bliss \& Lomo, 1973; Collingridge \& Bliss, 1987). There are two major methods to induce LTP in neuron cells. One way is to employ electrical stimulation; the other one is by using chemically induction. Chemically induced long-term potentiation (cLTP) has similar effects as the electrical one (Gu et al, 2010; Roth-Alpermann et al, 2006) but a larger proportion of synapses will be potentiated. In my thesis, I used chemically stimulation to induce cLTP.

As mention in section 1.1.3, the mechanism of LTP induction is that NMDAR serves as the initiator of the LTP process when the depolarized membrane potential is sufficient to remove $\mathrm{Mg}^{2+}$ from NMDAR. The opening of the $\mathrm{Mg}^{2+}$ gated ion channel in the early stimulation stage facilitates the elevation of the $\mathrm{Ca}^{2+}$ concentration. This $\mathrm{Mg}^{2+}$ blockage allows the flow of $\mathrm{Na}^{+}$and $\mathrm{Ca}^{2+}$ ions into, and $\mathrm{K}^{+}$out of the cell in a voltage-dependent manner. Moreover, the opening of the channel to facilitate $\mathrm{Ca}^{2+}$ flow into the cell is further promoted by the binding of glutamate to the receptors. A potentiation buffer that contains increased potassium ions, reduced magnesium ions and an extra addition of $25 \mathrm{mM}$ TEA was used to evoke cLTP. TEA has been known as a $\mathrm{K}^{+}$channel blocker that produces more depolarized membrane potential and therefore cLTP occurs. Morphological enlargement of dendritic spine is one criterion to judge whether the neuron cell have been potentiated.

A reconstructed maximum projection image from a series of optical sectioning images of CA3 hippocampal pyramidal neurons is shown in Figure 3.4.1(A). The image of dendritic spines in part of the apical domain of CA3 region (red rectangle area) labeled with fCherry was shown in Figure 3.4.1(B). Inducing cLTP with 25mM TEA, the morphological changes with a function of time are shown in Figure 3.4.1(C), where the morphological enlargements of dendritic spines after TEA stimulation were highlighted with orange arrows, while blue arrows indicate dendritic spines without morphological changes. The statistics of the change in spine head width are shown in Figure 3.4.2, in which the measurement of spine head diameter at different time points shows that it reaches its maximum after 20 minutes of TEA stimulation. The time point to measure actin dynamics after cLTP was set at 30 minutes after the treatment of TEA for the FCS experiments, as well as for the FRAP experiments. 
(A)

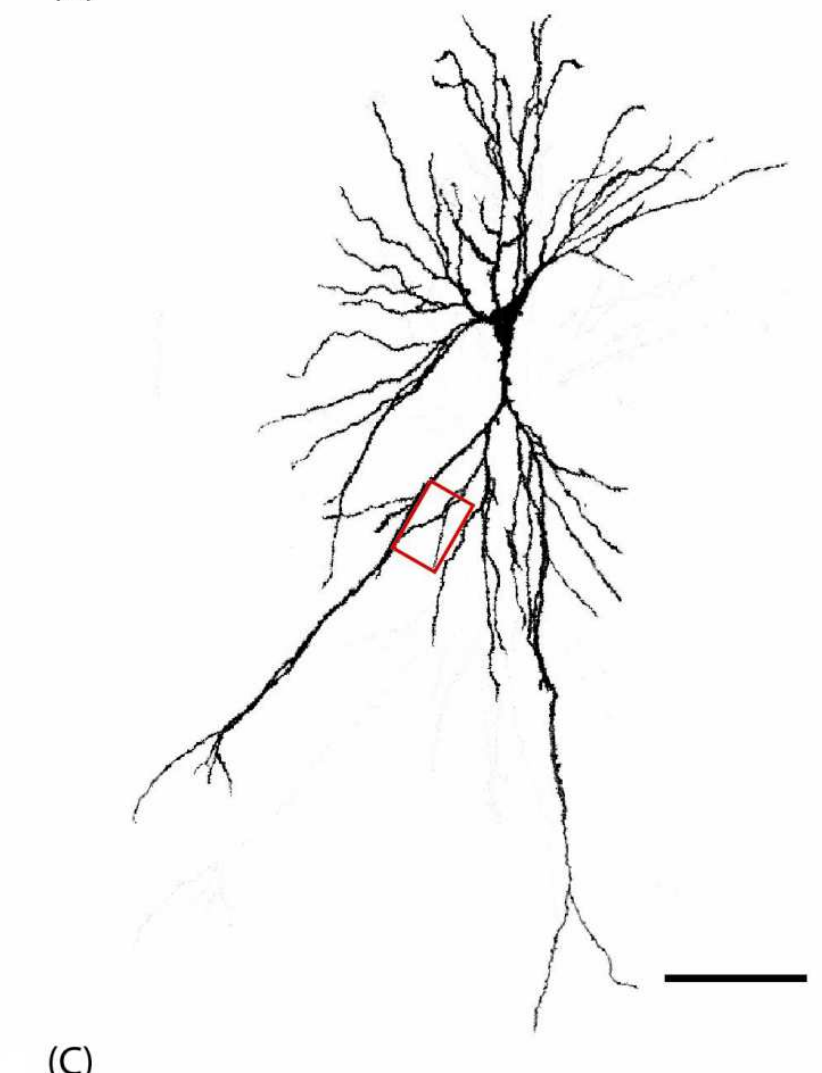

(C)

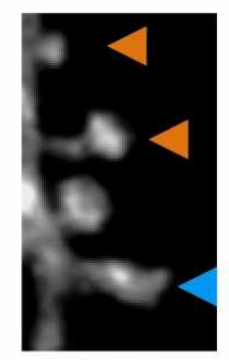

$-10 \min$

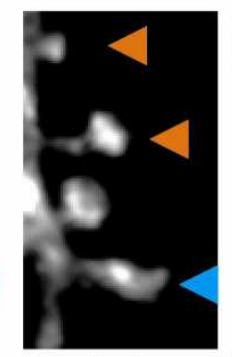

Omin

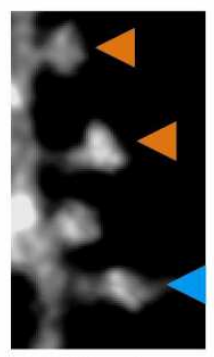

$10 \mathrm{~min}$

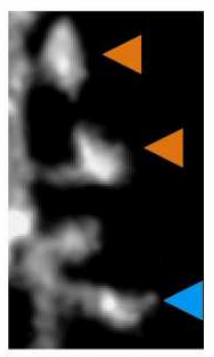

$20 \mathrm{~min}$
(B)
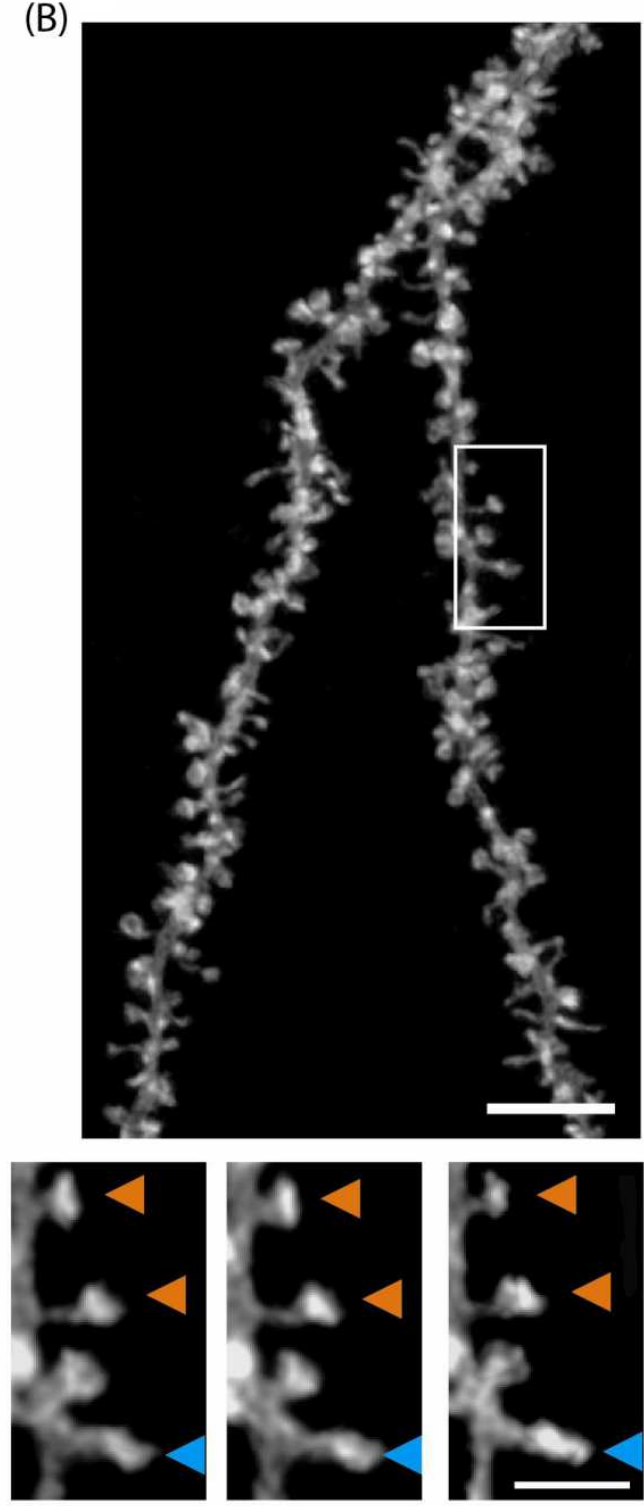

$30 \mathrm{~min}$

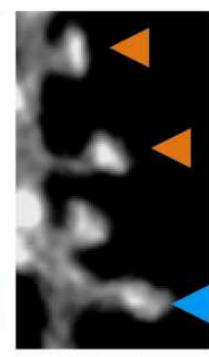

$40 \mathrm{~min}$

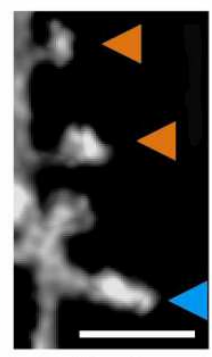

$50 \mathrm{~min}$

Figure 3.4.1 Dendritic spines in CA3 hippocampal pyramidal neurons and their morphological changes after cLTP. (A) Maximum projection image from a stack of optical sectioning images of CA3 hippocampal pyramidal neurons. (B) fCherry labeled dendritic spines in part of the apical domain of CA3 pyramidal cells (zoom in from red rectangle area in (A)). (C) TEA induced morphological changes in dendritic spines (zoom in from white rectangle area in (B)) with a function of time. The orange arrows point out the enlargement of dendritic spines and the blue arrow points out that the dendritic spine remains stable. (Scale bar: $50,5,2 \mu \mathrm{m}$, respectively)

(Data taken by Yves Kellner) 


\section{spine head width after cLTP}

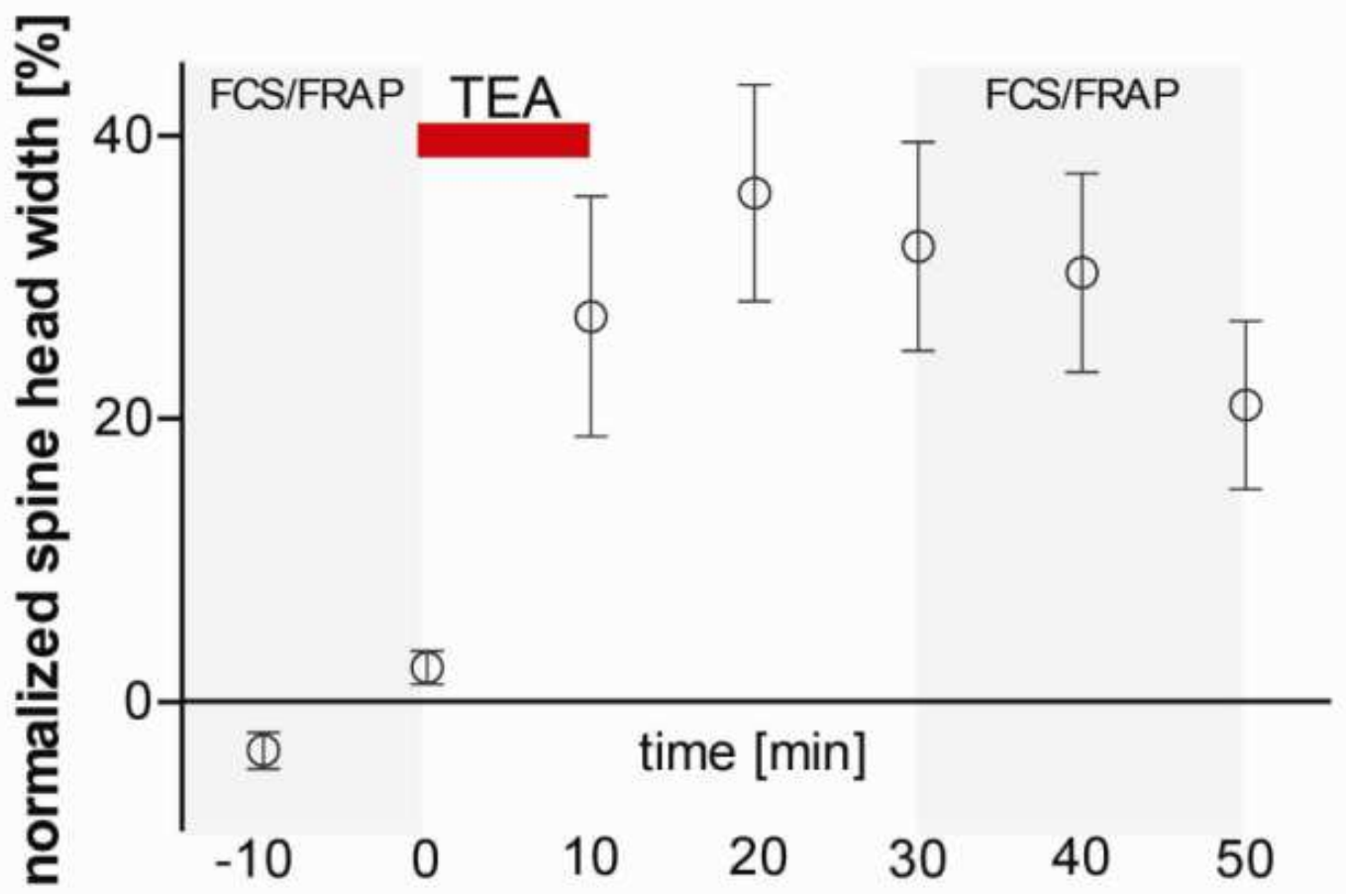

Figure 3.4.2 Statistics of morphological enlargement after cLTP. Morphological enlargement with the function of time shows that the increase of the spine width reached its maximum after 20 minutes. FCS and FRAP experiments were firstly performed before TEA stimulation while another round of experiments on actin dynamics after TEA stimulation was performed after 30 minutes. Error bars represent s.e.m.

(Data taken by Yves Kellner) 


\subsection{Actin dynamics with the function of cLTP}

The main goal of this study is to characterize the dynamic changes of actin filament due to cLTP induced morphological enlargement of dendritic spine. In this section, two different methods were applied to investigate actin dynamics with the function of TEA induced cLTP. The first approach was done by our collaboration partners and is based on fluorescence recovery after photobleaching (FRAP) and the second one is based on FCS. FRAP allows for differentiating the static and dynamic fractions of the actin filaments within the dendritic spine, since 2P-FCS only detects the dynamic fraction. The advantage of FCS measurements compared to FRAP is that the different sub-populations of dynamic actin filaments can be further distinguished.

(A)
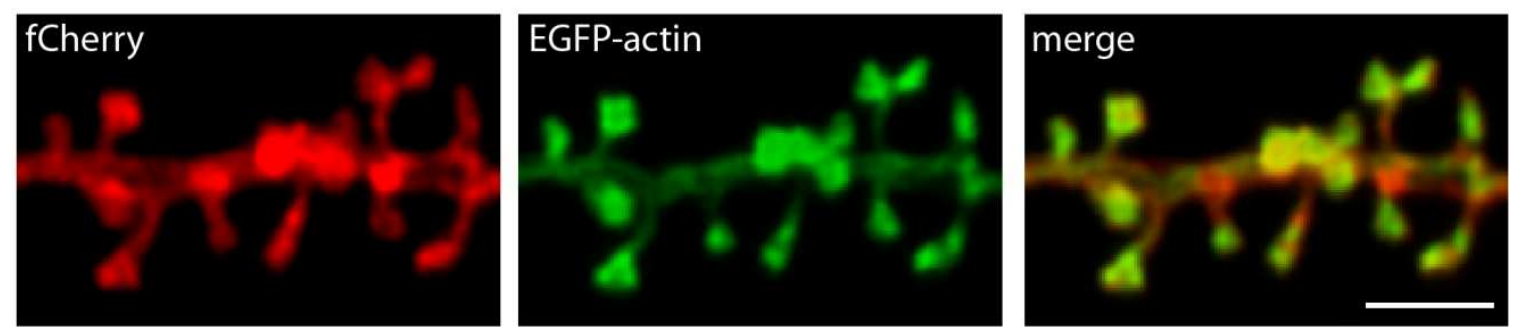

(B)

(C)
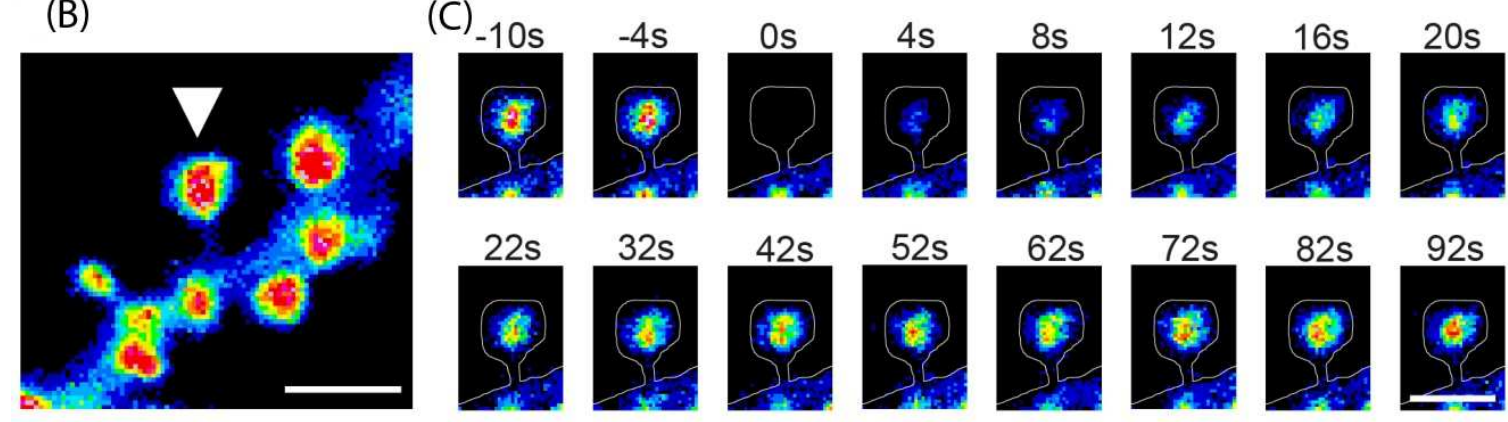

Figure 3.5.1 Fluorescence recovery after photobleaching (FRAP) imaging of eGFP-actin within dendritic spines. (A) Images of fCherry labeled neuron cells, eGFP-actin labeled dendritic spine and the merged image, respectively. The merged image shows that most of the actin structures are accumulated in spine heads. (B) Pseudo-color encoded fluorescence intensity image of eGFP-actin before photobleaching shows that actin molecules are concentrated in the spine head. (C) Intensity recovery of eGFP-actin after photobleaching in a single dendritic spine (pointed out by a white arrow in (B))(Scale bar: $2 \mu \mathrm{m}$ )

(Data taken by Yves Kellner) 


\subsubsection{Actin dynamics within dendritic spine studied by FRAP}

All the FRAP experiments* were performed between 15-19 DIV at single spines of secondary or tertiary dendritic branches of CA3 hippocampal neurons. For both morphological and dynamic observation, the CA3 hippocampal neurons were co-transfected with fCherry and eGFP-actin. The images shown in Figure 3.5.1(A) represent fCherry labeled dendritic spines, eGFP-actin labeled dendritic spines and the merged image, respectively. Data shown in Figure 3.5.1 (B) is pseudo-color encoded to present the fluorescent intensity of eGFP-actin, from which one can find that actin molecules are concentrated in the area of the spine head. The recovery of fluorescence intensity after photobleaching within a single dendritic spine is shown in Figure 3.5.1 (C). Due to dynamic mobility of actin filaments, the intensity recovers with the function of observation time.

To compare dynamic changes of actin filaments, FRAP ${ }^{*}$ was performed before and after TEA stimulation. As shown in Figure 3.5.2, the statistics of intensity recovery was done by recording 16 and 19 single dendritic spines before and after TEA stimulation. Only dendritic spines exhibiting morphological enlargement after TEA stimulation were selected for experiments. Due to the equilibrium diffusion of actin molecules within the dendritic spine head, the fluorescent signal will recover. Based on this method, actin molecules with different mobility can be distinguished and classified as three groups: stable actin pool, dynamic actin pool and monomeric G-actin pool.

In Figure 3.5.2(A), one can find that the recovery fluorescence intensity (red curve) in these enlarged dendritic spines is significantly higher than that in the corresponding control experiments (blue curve). By analyzing the difference of recovery curves, the changes in actin compositions before and after TEA stimulation can be compared. The induction of cLTP leads to a decrease of the stable pool actin filaments from $21.9 \pm 3.3 \%$ to $10.2 \pm 1.5 \%$ and an increase of the dynamic pool actin filaments from $64.2 \pm 3.3 \%$ to $78.6 \pm 2.2 \%$. Neither the Gactin pool nor the turnover time is changed significantly. The comparison of the changes in the composition of actin pools and turnover time are listed in Table 3.2.

\footnotetext{
* Experiments were done by Y.K.
} 


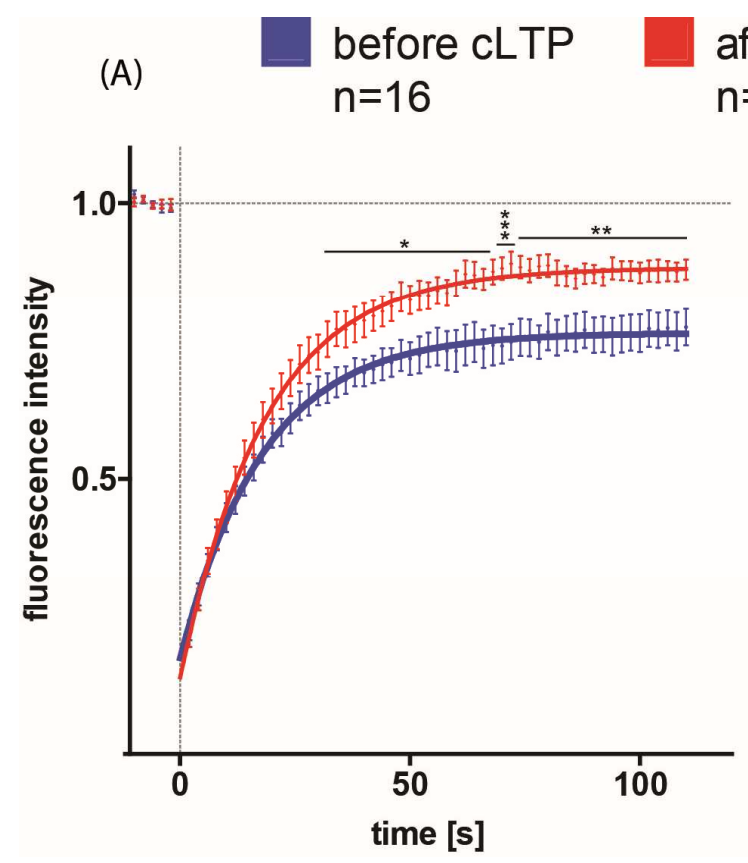

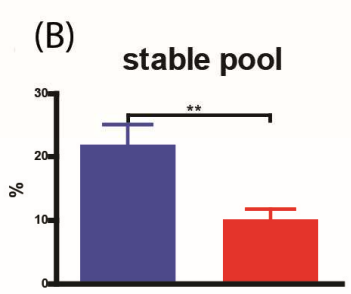

(D) monomeric pool

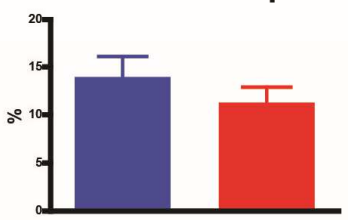

(C) dynamic pool

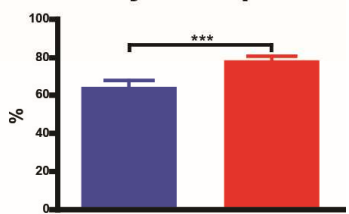

(E) turnover time

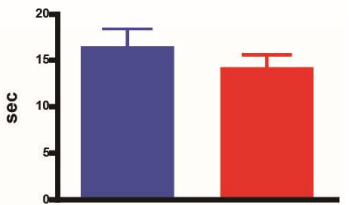

Figure 3.5.2 Fluorescence recovery after photobleaching (FRAP) analysis of eGFP-actin dynamics before and after cLTP. (A) Fluorescence recovery curves for eGFP-actin at single spines after photobleaching, which is performed before (blue curve) and after (red curve) the induction of cLTP. The recovery curves show a shift towards faster actin dynamics and a reduction of the stable F-actin. (B) The decrease of stable actin pool after cLTP $(p=0.0021)$, (C) The increase of dynamic actin pool after cLTP $(\mathrm{p}=0.0008)$. (D) The changes in the Gactin pool are not significant. (E) The changes in turnover time are not significant.

(Data taken by Yves Kellner)

Table 3.2 Comparisons of the changes in different actin pools and turnover time before and after cLTP acquired with FRAP measurements.

\begin{tabular}{|c|c|c|}
\hline Actin pools: & Before cLTP & After cLTP \\
\hline Stable pool (\%) & $21.9 \pm 3.3$ & $10.2 \pm 1.5$ \\
\hline Dynamic pool (\%) & $64.2 \pm 3.3$ & $78.6 \pm 2.2$ \\
\hline G-actin (\%) & $13.9 \pm 2.1$ & $11.2 \pm 1.6$ \\
\hline Turnover time (sec) & $16.5 \pm 1.8$ & $14.3 \pm 1.5$ \\
\hline
\end{tabular}




\subsubsection{Actin dynamics within dendritic spine studied by 2P-FCS}

The FRAP* results suggest that shortly after cLTP induction the actin cytoskeleton becomes more dynamic and may be correlated to morphological changes. Furthermore, these results demonstrate that cLTP influences the structural compositions of the actin cytoskeleton within dendritic spines, with the amount of stable actin filaments being decreased and dynamic actin filaments being increased. In the next step, FCS was used to quantify how the dynamic pool of actin filaments changed after cLTP. In total 200 spines were investigated but only those spines that did neither exhibit movements of the entire spine head before nor after cLTP were used for further analysis. This selection ensures that only intra-spine actin dynamics are analyzed without any bias from movements of the entire spine head. For FCS measurement, the two-photon excitation spot was placed in the center of different spine heads. With the same stimulation procedure as in FRAP experiment, the comparison of actin dynamics in FCS experiments were done by paired measurements only if dendritic spines underwent morphological enlargements after cLTP. Figure 3.5.3 shows those spines that displayed a significant growth in size $(29.48 \pm 2.19 \%)$. In Figure 3.5.3(B), comparisons of normalized autocorrelation curves depict the significant faster dynamics after cLTP.

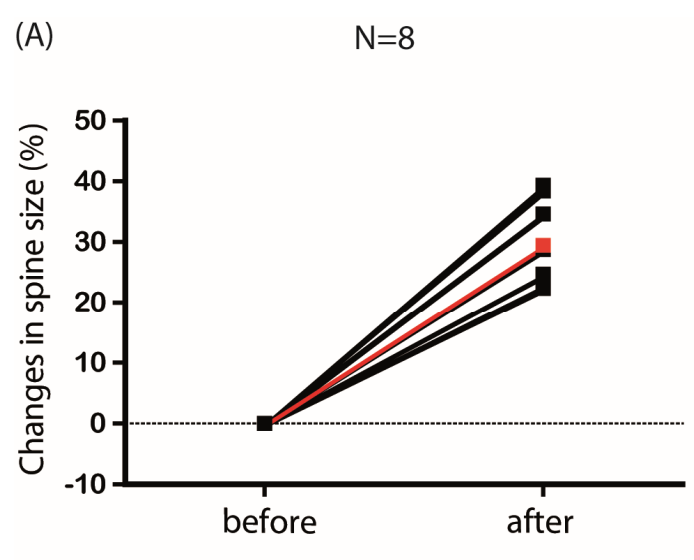

TEA stimulation

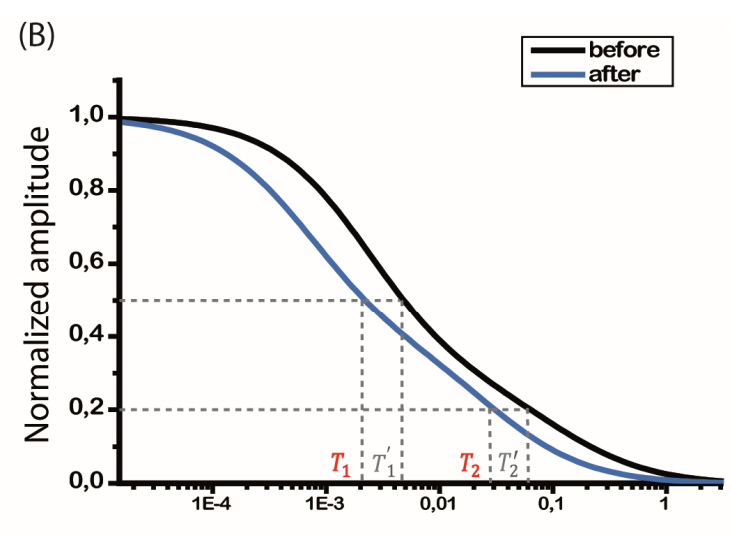

Lag time (sec)

Figure 3.5.3 Morphological changes of dendritic spines and the changes in actin dynamics before and after cLTP. (A) Morphological changes of dendritic spines $(\mathrm{N}=8)$ before and after TEA induced cLTP (Average values are shown in red) (B) Normalized FCS autocorrelation curves before (black curve) and after (blue curve) TEA induced cLTP.

\footnotetext{
${ }^{*}$ Experiments were done by Y.K.
} 
Dynamic and composition changes of actin filaments before and after cLTP are shown in Figure 3.5.4. After cLTP induced morphological enlargement, the diffusion mobility of both actin groups, short-form and long-form F-actin, was faster in the 8 measured spines. The diffusion time of short-form F-actin decreased from $[0.0041 \pm 0.0006 \mathrm{~s}]$ to $[0.0017 \pm 0.0004 \mathrm{~s}]$ and that of long-form F-actin decreased from $[0.47 \pm 0.07 \mathrm{~s}]$ to $[0.18 \pm 0.05 \mathrm{~s}]$, which accounted for $64.5 \%$ and $71.3 \%$, respectively. Changes in diffusion mobility may imply structural modification. With changes in dynamics by a factor of 2-3 after cLTP, one can estimate that there is also a change in polymerization degree. Furthermore, the heterogeneity of actin filaments within these enlarged dendritic spines did also increase after cLTP, which is shown in Figure 3.5.4 (C) and (D).

Next, we fit the autocorrelation curves with the model of different brightness (section 3.2.2) in order to further quantitatively compare the changes in particle number and brightness after TEA stimulation. In Figure 3.5.5 (A), the changes in particle number of the short-form F-actin were not as significant as these in the long-form F-actin after cLTP $(-2 \pm 26 \%$ compared to $75 \pm 25 \%$ ), which may likely due to their faster diffusion mobility. In Figure 3.5.5(B), we observed a significant $75 \%$ increase of longer form F-actin within those enlarged dendritic spines that may result from the cutting of longer form actin filaments. This cutting effect implies the remodeling and reorganization of actin filaments within dendritic spine after cLTP. Furthermore, the decrease in particle brightness for both groups can also provide the evidence of the cutting effect after cLTP. In Figure 3.3.5 (C) and (D), the changes in particle brightness of short- and long-form F-actin are presented as follows: [1809 \pm 347 to $1588 \pm 332$ a.u.] and $\left[(117 \pm 30)^{*} 10^{3}\right.$ to $(78 \pm 22)^{*} 10^{3}$ a.u.], respectively. Furthermore, Figure 3.5.6 demonstrates that the change in overall intensity within the observation focal volume did not significant before and after TEA stimulation. A comparison of physical parameters before and after cLTP is listed in Table 3.3 . 
(A)

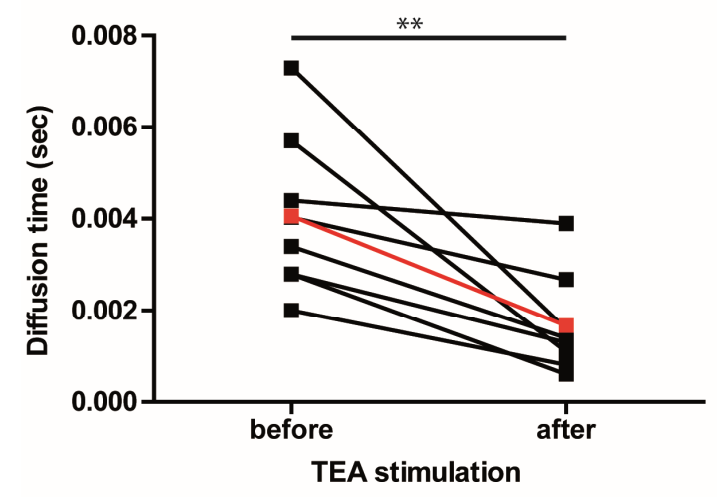

(C)

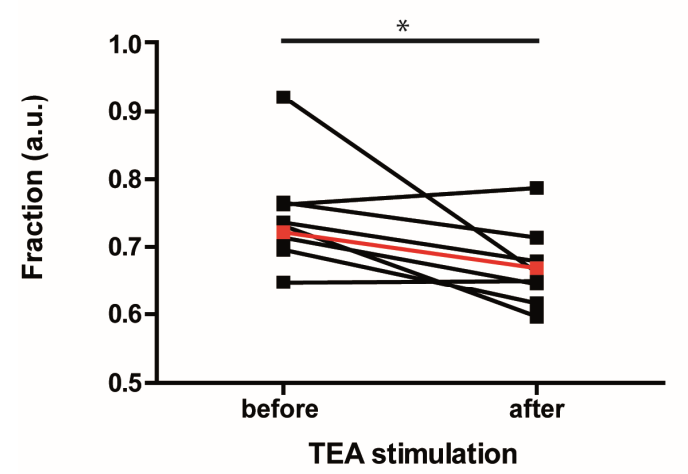

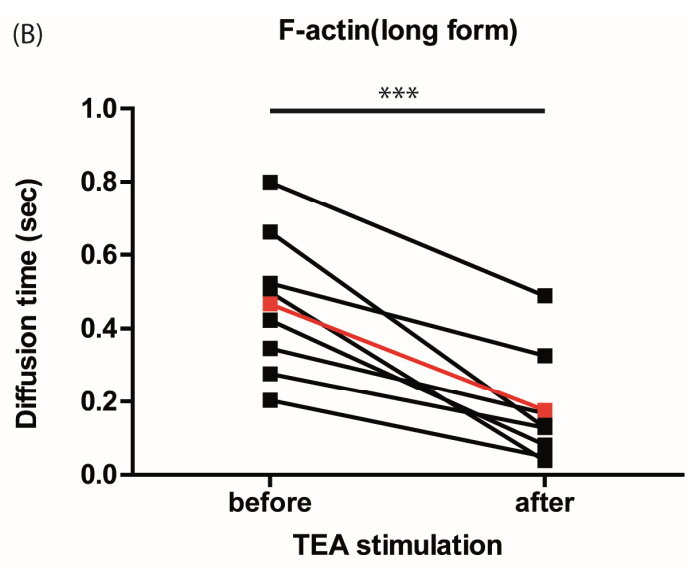

(D)

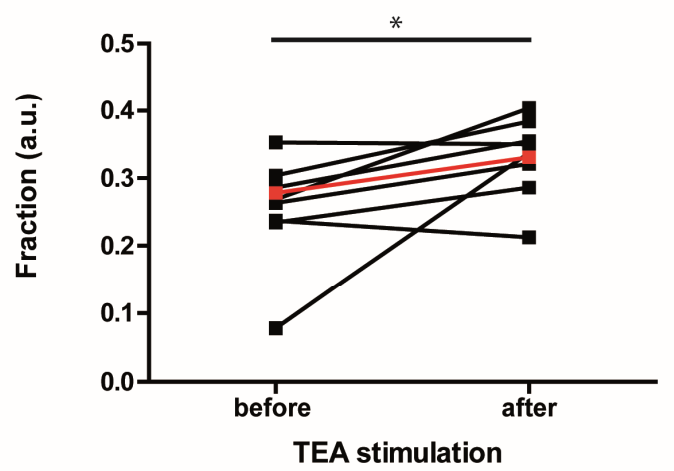

Figure 3.5.4 Changes in diffusion time and heterogeneity of actin filaments before and after cLTP. With TEA stimulation, actin filaments undergo cutting effect within enlarged dendritic spines, which results in faster diffusion dynamics of two major groups. (A) and (B) The diffusion time decreases in both short-form F-actin and long-form F-actin ( $p=0.0074$ and 0.0008, respectively); (C) The molecular fraction of short-form F-actin decreases ( $\mathrm{p}=0.0405$ ); (D) The molecular fraction of long-form F-actin increases (Average values are shown in red; the results of molecular fractions are derived from two-component fitting) 
(A)

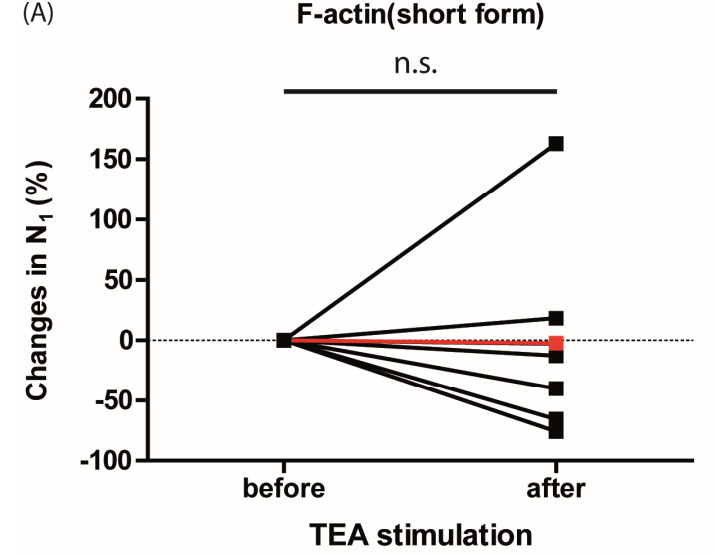

(C)

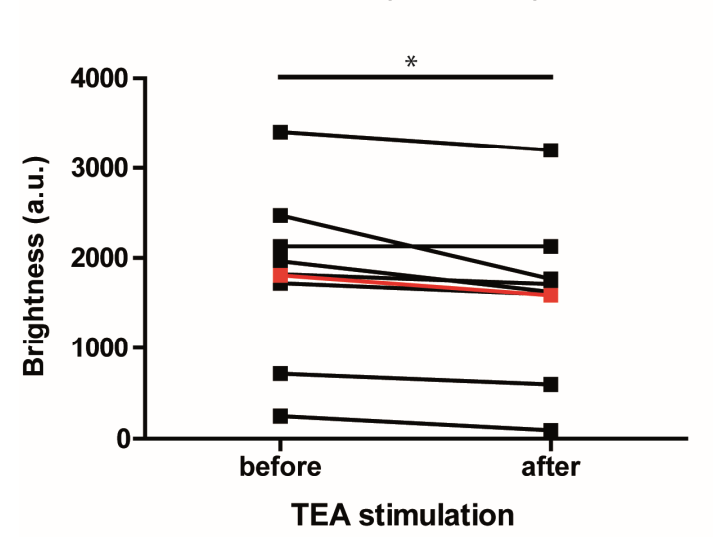

(B) F-actin(long form)

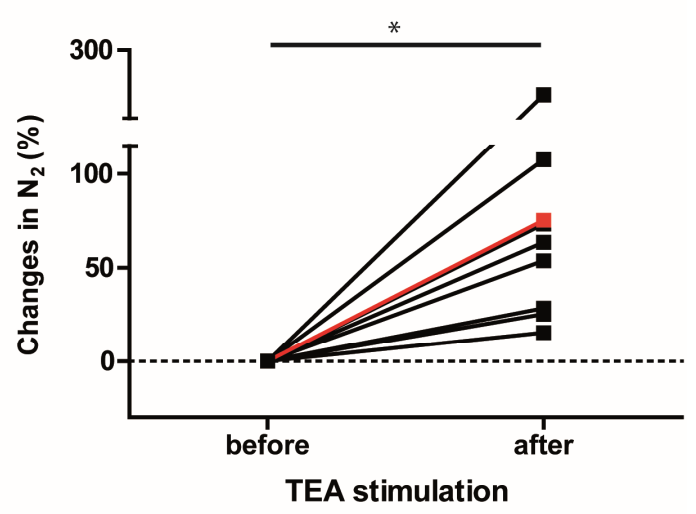

(D) F-actin(long form)

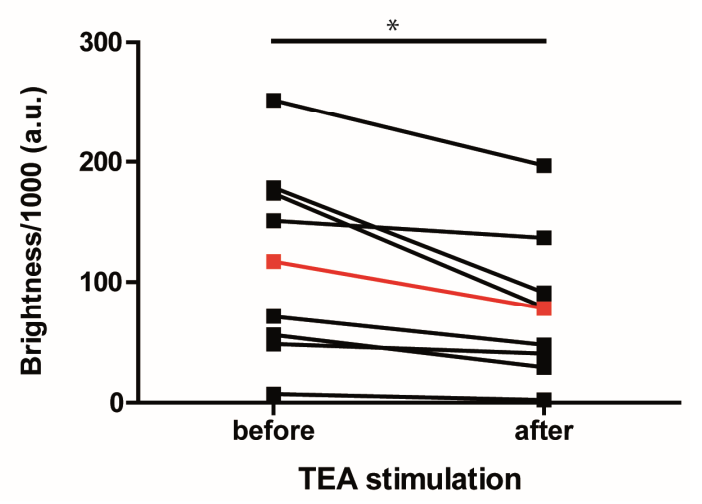

Figure 3.5.5 Changes of particle number and brightness within dendritic spines before and after cLTP. (A) Averaged particle numbers of the short-form F-actin is not altered significantly whereas in (B) that the averaged particle numbers of long-form F-actin increases $(p=0.0014)$. (C) and (D) represent the decrease of particle brightness after TEA stimulation in both short-form and long-form groups ( $\mathrm{p}=0.0243$ and 0.0168 , respectively). 


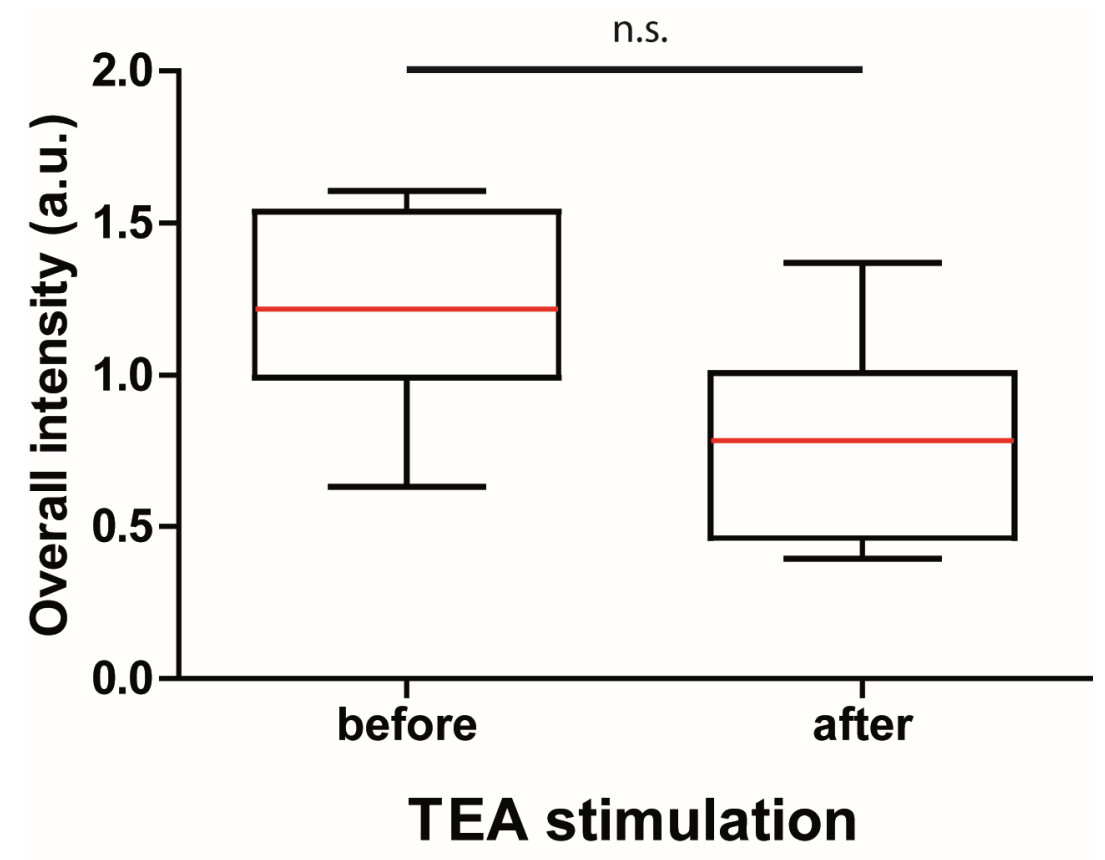

Figure 3.5.6 Changes in overall intensity before and after cLTP. The overall intensity within dendritic spines before and after cLTP is not decreased significantly, which implies that there is no significant decrease of molecular concentration within the observation focal volume.

Table 3.3 Summary of physical parameters of both groups before and after TEA stimulation.

\begin{tabular}{|c|c|c|c|}
\hline \multicolumn{2}{|c|}{} & Before TEA & After TEA \\
\hline \multirow{2}{*}{ Diffusion time (sec) } & Short-form F-actin & $0.0041 \pm 0.0006$ & $0.0017 \pm 0.0004$ \\
\cline { 2 - 4 } & Long-form F-actin & $0.47 \pm 0.07$ & $0.18 \pm 0.05$ \\
\hline \multirow{3}{*}{ Change in particle number (\%) } & Short-form F-actin & 0 & $-2 \pm 26$ \\
\cline { 2 - 4 } & Long-form F-actin & 0 & $75 \pm 25$ \\
\hline \multirow{2}{*}{ Particle brightness (a.u.) } & Short-form F-actin & $1809 \pm 347$ & $1588 \pm 332$ \\
\cline { 2 - 4 } & Long-form F-actin & $(117 \pm 30) * 10^{3}$ & $(78 \pm 22)^{*} 10^{3}$ \\
\hline
\end{tabular}




\subsection{Control experiments}

In order to test whether the changes of actin dynamics are related to the cLTP induced morphological enlargement of dendritic spines, several control experiments were performed to prove the contributions of actin filaments to morphological modulations. The first control experiment is to observe actin dynamics when the function of cLTP is blocked by treating the neuron with the NMDAR antagonist AP5 together with the L-type calcium channel blocker Verapamil (Roth-Alpermann et al, 2006). If cLTP shares the same pathway as electrical LTP, the inhibition of NMDAR and VDCC (voltage-dependent calcium current) should also inhibit potentiation. Secondly, although most of the dendritic spines undergo morphological changes in response to cLTP induction, a few parts of them remain stable. Experiments on spines without morphological changes after cLTP were performed to demonstrate the correlation between changes in the observed actin dynamics and the cLTP induced morphological enlargements. Moreover, neuron without any treatment was measured to investigate the consistency of intrinsic actin dynamics.

\subsubsection{Actin dynamics under cLTP with the treatment of AP5 and Verapamil}

Similar to the experimental sets, the first round of 2P-FCS measurements were performed with the neuron bathed in normal HEPES buffer. After these measurements, the buffer was replaced with TEA stimulation buffer including AP5 and Verapamil for 10 minutes. Afterwards, the solution was changed to AP5 and Verapamil containing HEPES recording buffer for another measurements.

Dendritic spines do not undergo morphological enlargement with the blockage of cLTP. Dendritic spines without significant changes in diffusion time of both actin groups before and after treatment as shown in Figure 3.6.1(A) and (B) implied that the actin structure did not significantly change. Compared with increased heterogeneity in enlarged dendritic spines, there is no significant change within these spines. Also, particle numbers did not change significant in long-form F-actin, as shown in Figure 3.6.4(A). For short-form F-actin, however, there was an obvious decrease in particle numbers. 


\subsubsection{TEA stimulation without morphological changes}

As shown in Figure 3.6.2, dendritic spines that did not show any significant morphological change after TEA stimulation did also not show any significant change in diffusion time and molecular heterogeneity. According to Figure 3.6.2(A) and (B), the changes in diffusion time of both actin groups are not significant within these stable dendritic spines. This result supports the idea that actin dynamics play a vital role in morphological modulation during the process of cLTP. Furthermore, based on Figure 3.6.2(C) and (D), the heterogeneity of molecular compositions did not change significantly before and after the stimulation. In Figure 3.6.4(B), the changes in particle numbers were not significant in both groups, either. Compared with the experimental sets of enlarged dendritic spines after cLTP, it can be concluded from this control experiment that the changes in actin dynamics and morphological enlargements are correlated. The remodeling and reorganization of actin filaments contribute to the morphological enlargements.

\subsubsection{Intrinsic actin dynamics without TEA stimulation}

Actin filaments are always in a dynamic equilibrium state to maintain diverse cellular function. The control experiment in this part focuses on the intrinsic dynamics of actin filaments within dendritic spines. The experiments were performed in 10 minutes intervals without any specific treatments to compare the differences of actin dynamics with the function of time.

As shown in Figure 3.6.3, using the same parameters as in previous experiments, the intrinsic actin dynamics show a time dependent consistency. Neither the diffusion time of both actin groups, nor the molecular heterogeneity change significantly. In addition, changes in particle numbers were not significant in both groups, as shown in Figure 3.6.4(B). The results represent that intrinsic actin dynamics were at steady-state equilibrium. This control experiment again proved that the dynamic actin filaments contribute to the morphological enlargements. Furthermore, Figure 3.6.5 demonstrates that the moderate changes of overall intensity among these control experiments. 


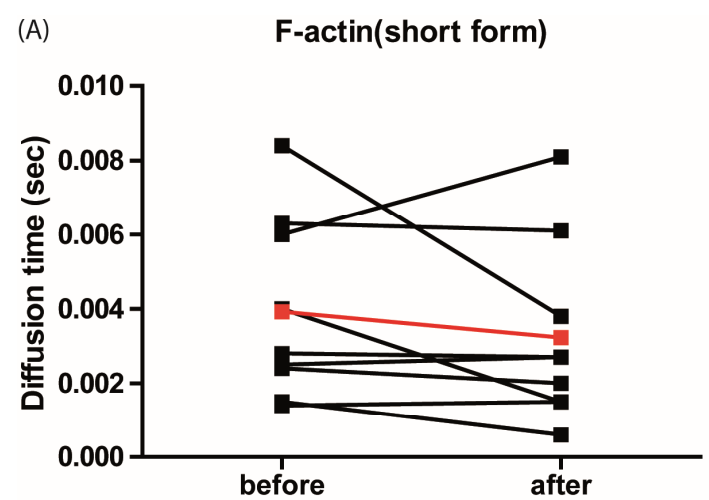

TEA+AP5+Verapamil

(C)

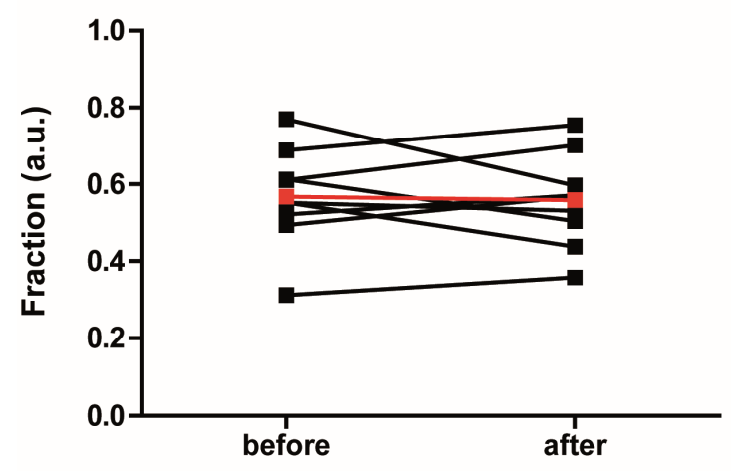

TEA+AP5+Verapamil

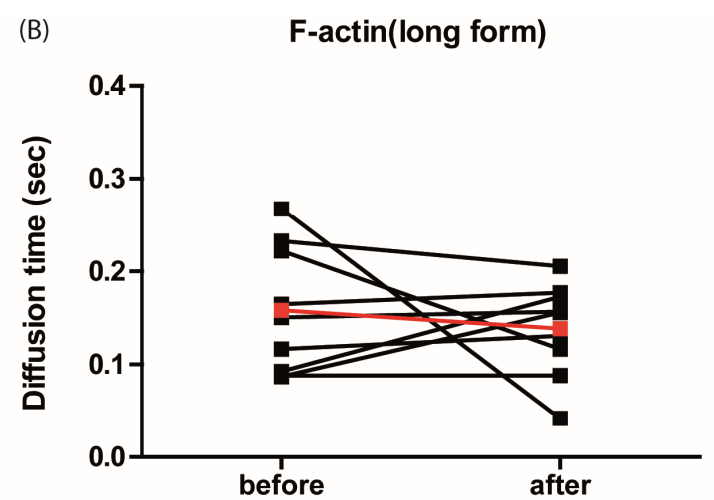

TEA+AP5+Verapamil
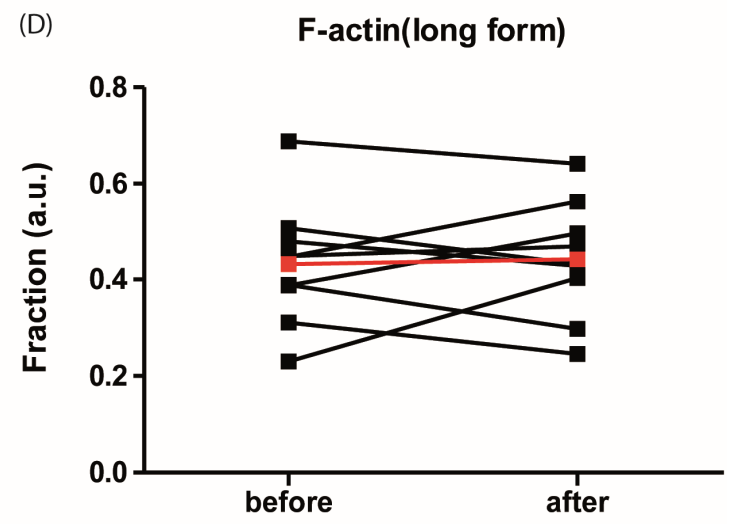

TEA+AP5+Verapamil

Figure 3.6.1 Changes in diffusion time and molecular heterogeneity with blockage of cLTP. There is no significant change in diffusion time and molecular heterogeneity after the blockage of cLTP (N=8). (A) and (B) The changes in diffusion time of two actin groups before and after the blocking of cLTP. (C) and (D) The changes in molecular heterogeneity of two actin groups before and after the blocking of cLTP. 


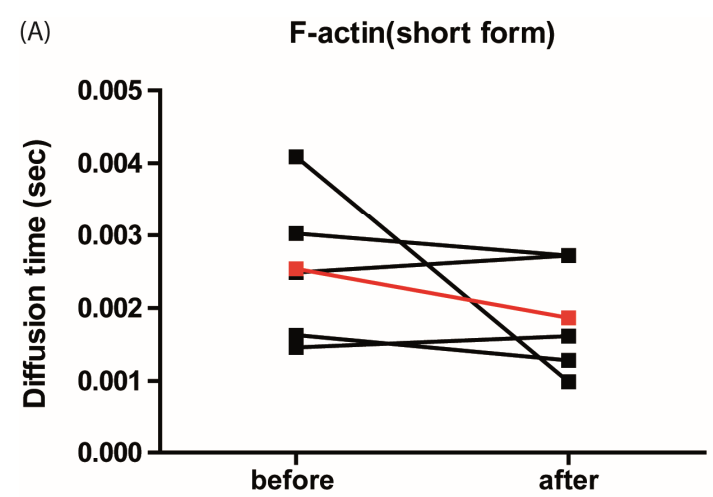

Stimulation without morphological change

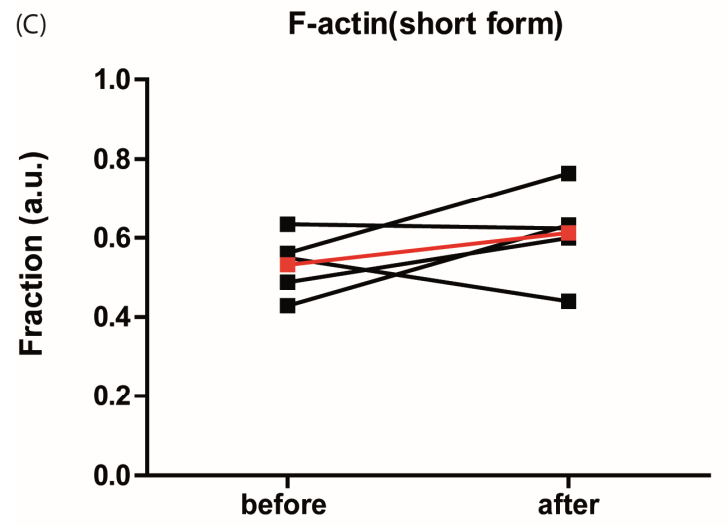

Stimulation without morphological change

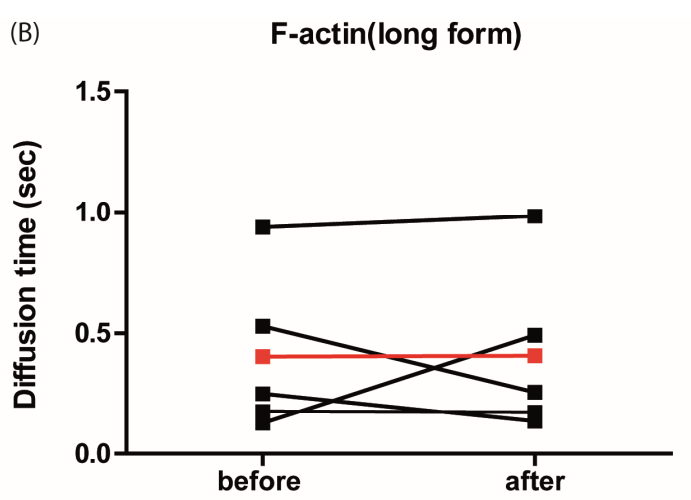

Stimulation without morphological change

(D) F-actin(long form)

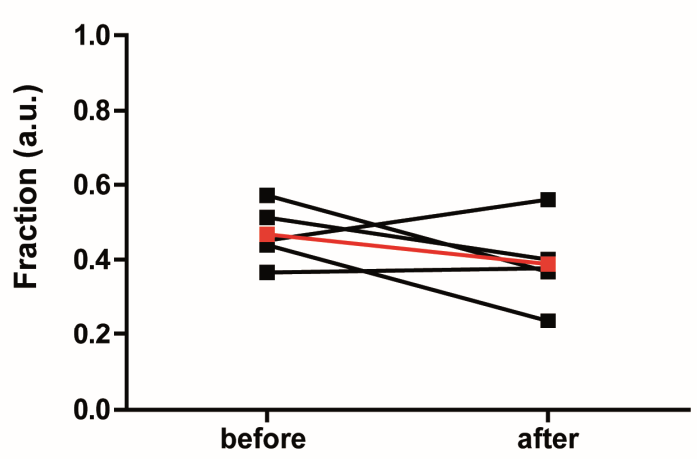

Stimulation without morphological change

Figure 3.6.2 Changes in diffusion time and molecular heterogeneity within dendritic spines without morphological changes with TEA treatment. There is no significant change in diffusion time and molecular heterogeneity within stable dendritic spines (N=5). (A) and (B) The changes in diffusion time of two actin groups within stable dendritic spines. (C) and (D) The changes in molecular heterogeneity of two actin groups within stable dendritic spines. 


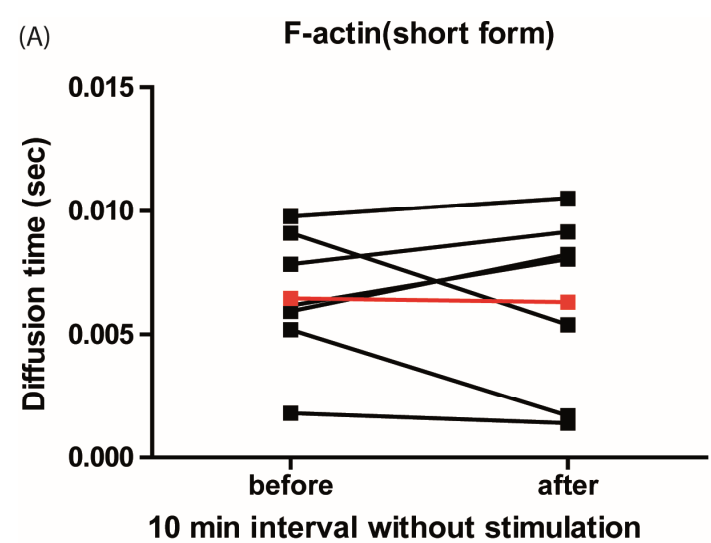

(C)

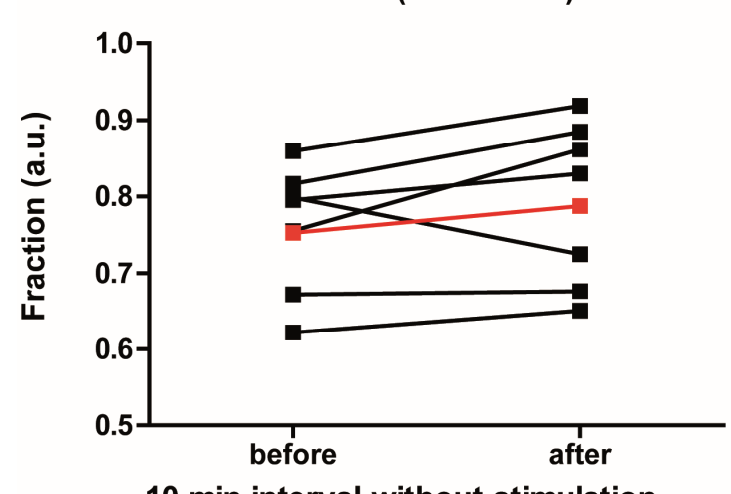

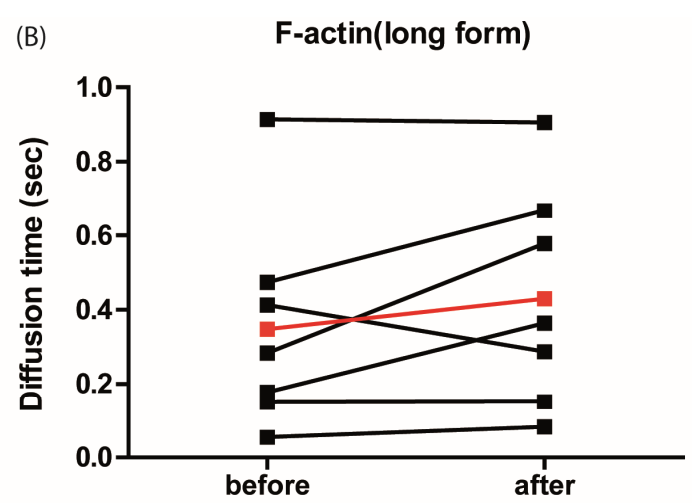

10 min interval without stimulation

(D) F-actin(long form)

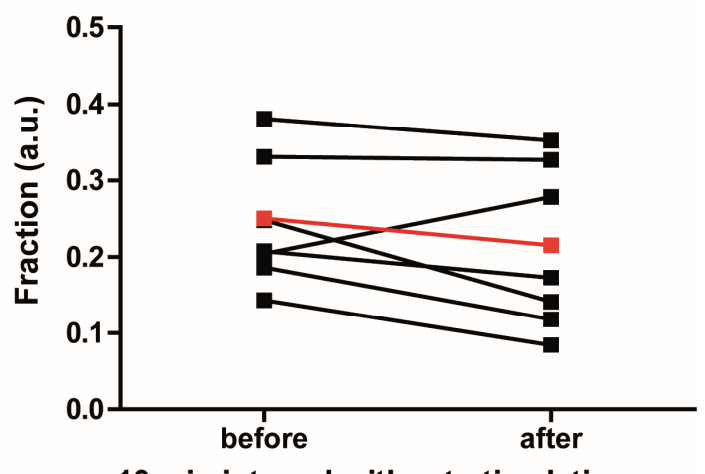

10 min interval without stimulation

Figure 3.6.3 Intrinsic actin dynamics and molecular heterogeneity without any treatment. There is no significant change in diffusion time and molecular heterogeneity within measured dendritic spines ( $N=7)$. (A) and (B) The changes in diffusion time of two actin groups within measured dendritic spines. (C) and (D) The changes in molecular heterogeneity of two actin groups within measured dendritic spines. 
(A)

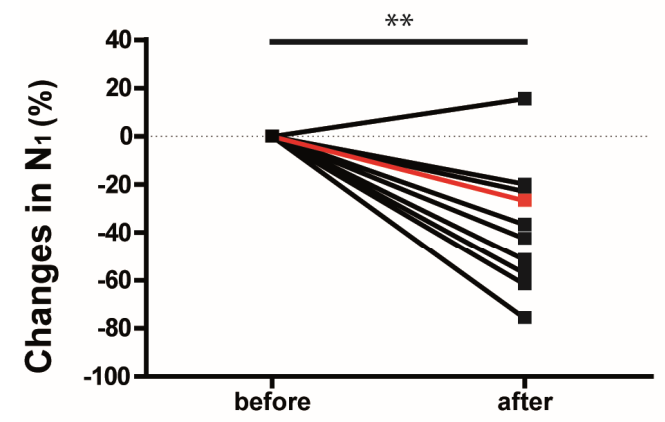

F-actin(long form)

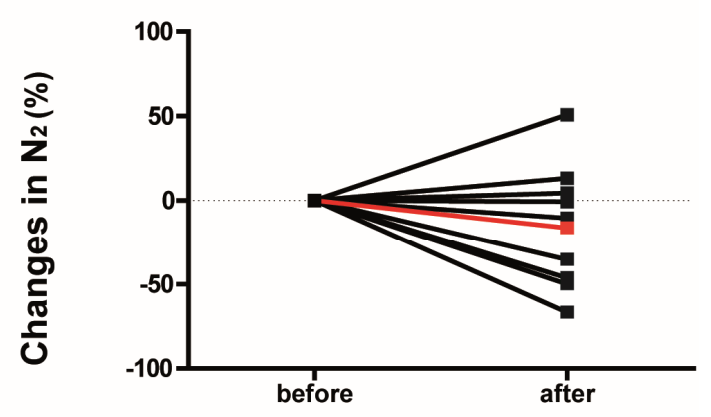

TEA+AP5+Verapamil

(B)

$$
\text { F-actin(short form) }
$$

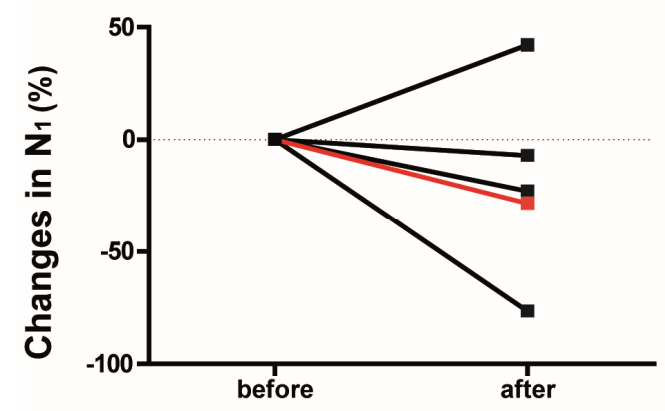

F-actin(long form)

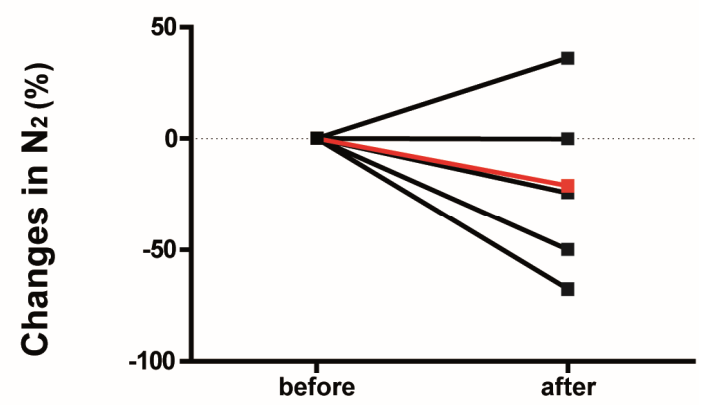

Stimulation without morphological change

(C)

$$
\text { F-actin(short form) }
$$

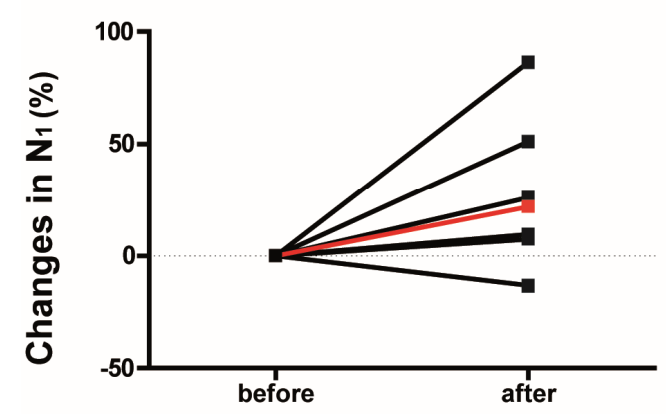

10 min interval without stimulation
F-actin(long form)

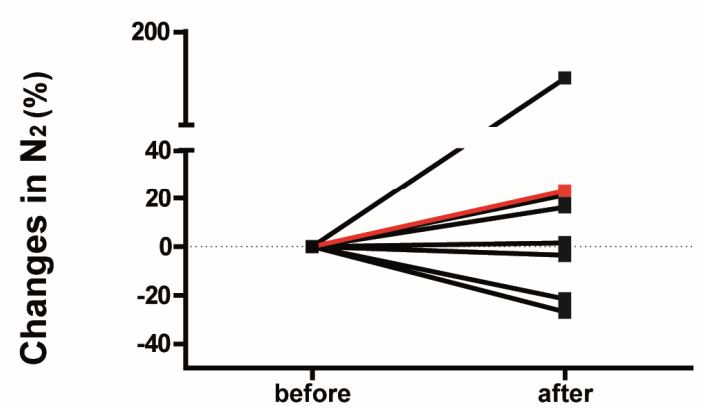

Figure 3.6.4 Changes in particle numbers in control experiments. The changes of particle number of both groups in the control experiments. (A) cLTP blocked by AP5 and Verapamil, (B) TEA stimulation without morphological changes and (C) Experiments measured in 10 min interval without stimulation. 


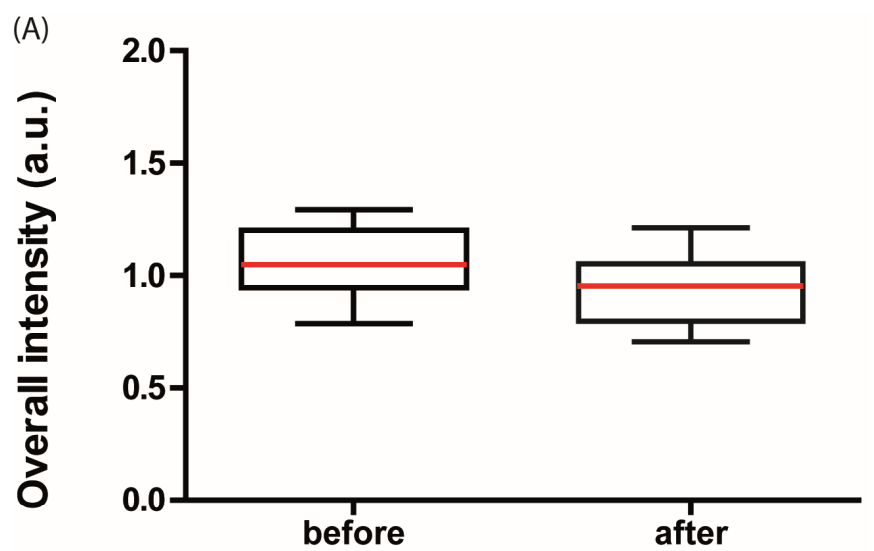

TEA+AP5+Verapamil

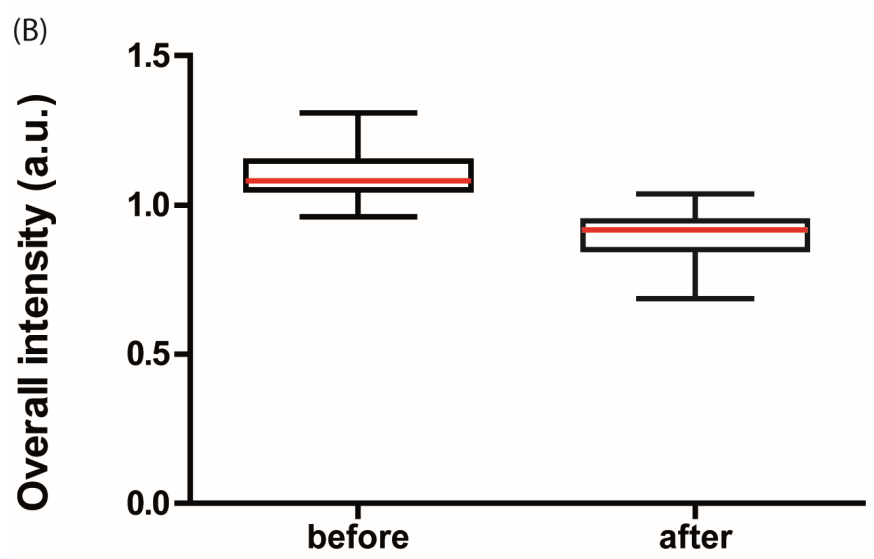

Stimulation without morphological change

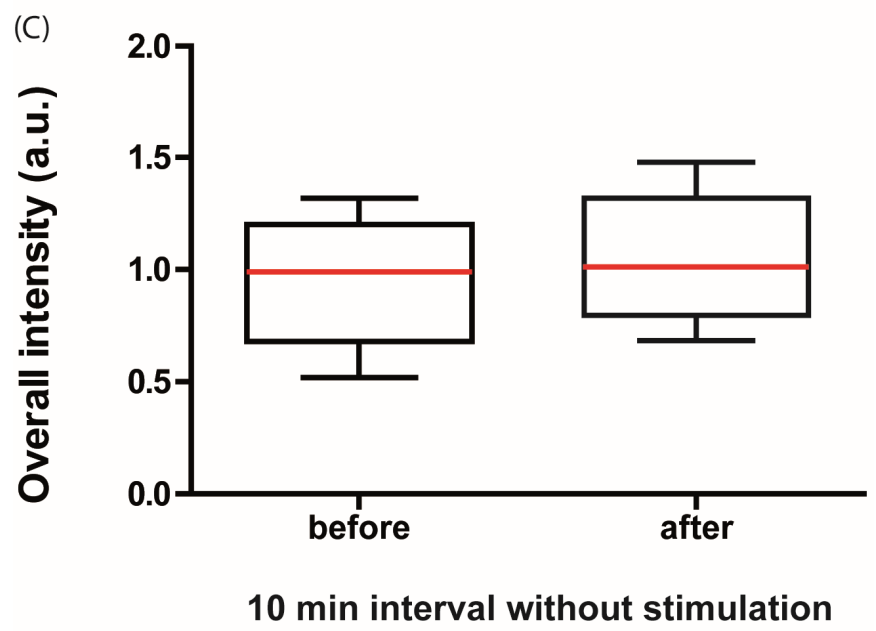

Figure 3.6.5 Changes in overall intensity in control experiments. (A) cLTP blocked by AP5 and Verapamil, (B) TEA stimulation without morphological changes and (C) Experiments measured in $10 \mathrm{~min}$ interval without stimulation. 


\subsection{Summary of molecular dynamics within living cells}

We have observed the heterogeneous dynamics of actin filaments within living cells by use of FRAP* and FCS (section 3.5). Actin filaments play a vital role in modulating structural or functional changes of living cells. Morphological changes or movements of living cells rely largely on actin filaments (Pollard \& Borisy, 2003), and therefore, most of the actin filaments are highly dynamic in order to support rapid changes within living cells (Honkura et al, 2008; Star et al, 2002). Due to their great importance, actin molecules are of great research interest in the fields of biochemistry and biophysics.

To begin with, using the FRAP method*, G-actin, mobile dynamic actin filaments and immobile stable actin filaments can already be distinguished (Figure 3.5.2). However, dynamics spanning orders of magnitude cannot be fitted from a single fluorescent intensity recovery curve. In earlier studies, monomeric actin molecules (molecular weight of eGFPactin $\sim 75 \mathrm{kDa}$ ) reach their equilibrium rapidly within 2 seconds (Star et al, 2002) owing to that fast molecular exchanging between dendritic spine and shaft (Svoboda et al, 1996). Therefore, the $\sim 10$ times slower turnover time mentioned in section 3.5.1, which is about 15 seconds, was mostly contributed by mobile dynamic actin filaments.

In my experiment, two-photon fluorescence correlation spectroscopy (2P-FCS) was used to characterize molecular dynamics within the dynamic pool of actin filaments. The key advantage of this approach is that it allows identification of different sub-populations within this dynamic actin pool, which is only observed as one averaged population in conventional FRAP experiments. From FCS measurements, we are able to characterize the dynamic part of actin filaments into two groups, short-form F-actin ( 10 actin monomers) and long-form Factin ( 1000 actin monomers), with diffusion coefficients differing by more than 100 times ( $\sim 7$ and $\sim 0.07 \mu \mathrm{m}^{2} / \mathrm{s}$, respectively). The FRAP experiment, while able to separate three different actin pools, does not provide quantitative information about different populations within the dynamics pool. Prior to the FCS measurements on actin filaments, the calibration is necessary and is performed using freely diffusing Annexin V-eGFP molecule within the twophoton focal volume determined previous with R6G. The diffusion coefficient of Annexin VeGFP is in line with the literature (Dayel et al, 1999; Partikian et al, 1998; Stasevich et al, 2010).

\footnotetext{
${ }^{*}$ Experiments were done by Y.K.
} 
The differences in particle size and structural composition result in heterogeneously fluorescence fluctuations in FCS measurements (Figure 3.2.1). Due to the heterogeneous diffusion mobility of actin filaments (Frost et al, 2010; Honkura et al, 2008), autocorrelation curves were fitted using a two-component fitting model (equation (3.1) and (3.2)). The dynamic behavior of two actin sub-populations as well as the molecular heterogeneity can be analyzed in detail. However, owing to different particle brightness of these two sub-groups, molecular fractions derived from this model do not describe the absolute particle numbers precisely. Therefore, a fitting model, which takes account into the difference in brightness, was used to provide quantitative information on particle number and brightness. The significant decrease in particle brightness of both the short- and long-form F-actin (Figure 3.5.5) confirms the compositional changes of actin filaments after cLTP, which is correlated to the faster actin dynamics.

Compared with researches using FRET to study the dynamic equilibrium between Gand F-actin (Okamoto et al, 2004), a major advantage of our approach is the use of a single label. In FRET experiments, double transfected cells are mandatory and the cross-talk between donor and acceptor signals has to be taken into consideration. Moreover, quantitative analysis of the dynamic and structural heterogeneity of actin filaments is not available from the FRET experiment.

While acquiring the temporal information of actin dynamics with FCS, the changes in morphology of dendritic spine are recorded using wide-field fluorescence microscopy. In this study, the spatial resolution of wide-field fluorescence image is sufficient to observe the morphological changes since the dimensions of spine heads are around $1 \mu \mathrm{m}$. 


\subsection{Remodeling and reorganization of actin filaments during synaptic plasticity}

In this work, we focused on the remodeling and reorganization of actin filaments within dendritic spines enlarged after cLTP. The morphological changes were fund to be correlated to the functional changes (Holtmaat \& Svoboda, 2009; Nagerl et al, 2008; Yuste \& Bonhoeffer, 2001), and our results agree with the notion that, during synaptic plasticity, the remodeling and reorganization of actin filaments are responsible for the structural modulations.

With the FRAP experiments*, it had been demonstrated that the fraction of dynamic actin filaments was increased after cLTP (Figure 3.5.2). By the combination of 2P-FCS and FM, we were able to further quantify the changes in the dynamics and heterogeneity of such filaments, via analyzing the various parameters obtained, such as diffusion time, particle number and molecular brightness. According to the Rouse model (Rouse, 1953; Ruddies et al, 1993), the decrease of diffusion time in both actin sub-populations by a factors of 2 (Figure 3.5.4) corresponds to a proportional decrease in the polymerization degree. The significant reduction of particle brightness in short-form and long-form F-actin after cLTP (Figure 3.5.5) confirmed the reductions of polymerization degree of actin filaments. Briefly, there is more dynamic shorter form F-actin within enlarged dendritic spine after cLTP that is in line with earlier observations that the dynamic actin filaments within dendritic spines are necessary for stable LTP (Krucker et al, 2000; Tatavarty et al, 2009).

The two sub-populations of mobile actin filaments consist of $\sim 10$ and $\sim 1000$ actin monomers, respectively, and were both shortened within enlarged dendritic spine after cLTP. This information is not available from FRAP or FRET measurements (as described in section 3.7). On the other hand, highly heterogeneous short actin filaments can be visualized by electron microscopy (EM) (Korobova \& Svitkina, 2010). However, it is not possible to monitor dynamic changes of actin filaments using EM.

Using the two-component fitting model considering different brightness, the shift of equilibrium towards shorter F-actin after cLTP is evidenced by the increased particle numbers. Furthermore, the reduced particle brightness yields the same conclusion. Comparing with drastically decreased overall intensity after Lat-A treatment (Frost et al, 2010; Honkura et al,

\footnotetext{
${ }^{*}$ Experiments were done by Y.K.
} 
2008; Okamoto et al, 2004), dendritic spine treated with TEA did not undergo significant intensity losses (Figure 3.5.6) which ensured the constant concentration of actin molecules confined within enlarged spine heads after cLTP.

In sum, taken into account the conserved overall concentration of actin molecules, the 2-3 times faster diffusion mobility, the reduced particle brightness and the increase particle numbers, we conclude that the actin filaments within dendritic spines, especially the longform ones, were cut shorter after cLTP. These shortened actin filaments may serve as the driving force or support backbones to facilitate morphological enlargement during LTP. Compared with other techniques, our FCS measurements provided detailed information regarding to the heterogeneous molecular mobility and the structural reorganization of actin filaments. 


\section{Conclusions}

First of all, from a technical point of view, the combination of two-photon fluorescence correlation spectroscopy (2P-FCS) with conventional one-photon wide field fluorescence microscopy (FM) and by using two different labels - one for the cellular morphology as detected by FM and another one for the very localized specifically observation of distinct proteins within the two-photon volume of $\sim 1 \mathrm{fl}-$ permits us to study simultaneously in high detail and resolution (bio)-molecular processes within selected dendritic spines. The penetration abilities of two-photon excitation allows us to focus on the tiny single dendritic spine with precision and thus to record the fluorescence fluctuation within this tiny focal volume (sub-micron level). The technical approach of our set-up observing the overall living cell structure while simultaneously investigating processes on a (bio)-molecular level at distinct and selected spots - can be easily applied to research fields other than neurobiology.

This approach was used in my thesis to decipher the dynamic properties and population changes of different classes actin filaments within the dendritic spine that occur during morphological changes induced by chemical long term potentiation (cLTP). The key advantage of our approach is that it allows identifying different sub-populations within the dynamic fraction of actin filaments that was previously only observed as one averaged population in conventional FRAP experiments. As expected, the experiments showed that morphological enlargements of dendritic spines due to the TEA induced cLTP are highly correlated to the dynamic changes of actin filaments.

It turned out that two-component fitting models are sufficient to describe quantitatively the observed FCS data. Thus, two groups of actin filaments with different dynamic mobility can be clearly distinguished and characterized. The different diffusion times observed from these fractions suggest an average size of $\sim 10$ and $~ 1000$ actin monomers, respectively. Both fractions became much more dynamic within spine heads upon cLTP but only when a morphological enlargement occurred. Surprisingly, the data showed that the number of long-form actin filaments increased significantly in the observation volume while the overall number of short-form filaments does at least not increase, as demonstrated by the non-significant change in the overall fluorescence intensity. This together with the additional fact that the diffusion time of the long-form F-actin decreases two to three times suggests that 
the longer actin filaments are cut into shorter filaments rather than simply being depolymerized. Of course, in total the final structure contains more actin molecules than before since the spine head grows.

Based on these observations, the model shown in Figure 5.1.1 is proposed for the actin reorganization and remodeling during cLTP within enlarging dendritic spines. The left and right parts show only the static fraction of the actin cytoskeleton before and after TEA induced morphological enlargement. The middle upper and lower parts show the population numbers as well as fragment sizes of the dynamic fraction of actin filaments as suggested by the 2P-FCS observation during cLTP. The red circular area within spine head indicates the volume that is actually observed in 2P-FCS and that remains constant during the growth of the spines. The 2P-FCS data suggest that the longer dynamic actin filament population is cut in somewhat smaller fragments of higher number. This higher number of long-form actin filaments can then be used to reorganize and extent the spine head in a similar fashion as a tent, which is pitched by tent poles that can be used as the backbones to support morphological enlargements. To sum up, we have observed more dynamic shorter branched actin filaments within enlarged dendritic spines after cLTP that may probably provide the exertion forces for the expansion of spine heads. The disruption of long-form F-actin into short-form F-actin may further support the morphological enlargement by using these fragments for a "tree-growing-like" behavior of the actin structure. 


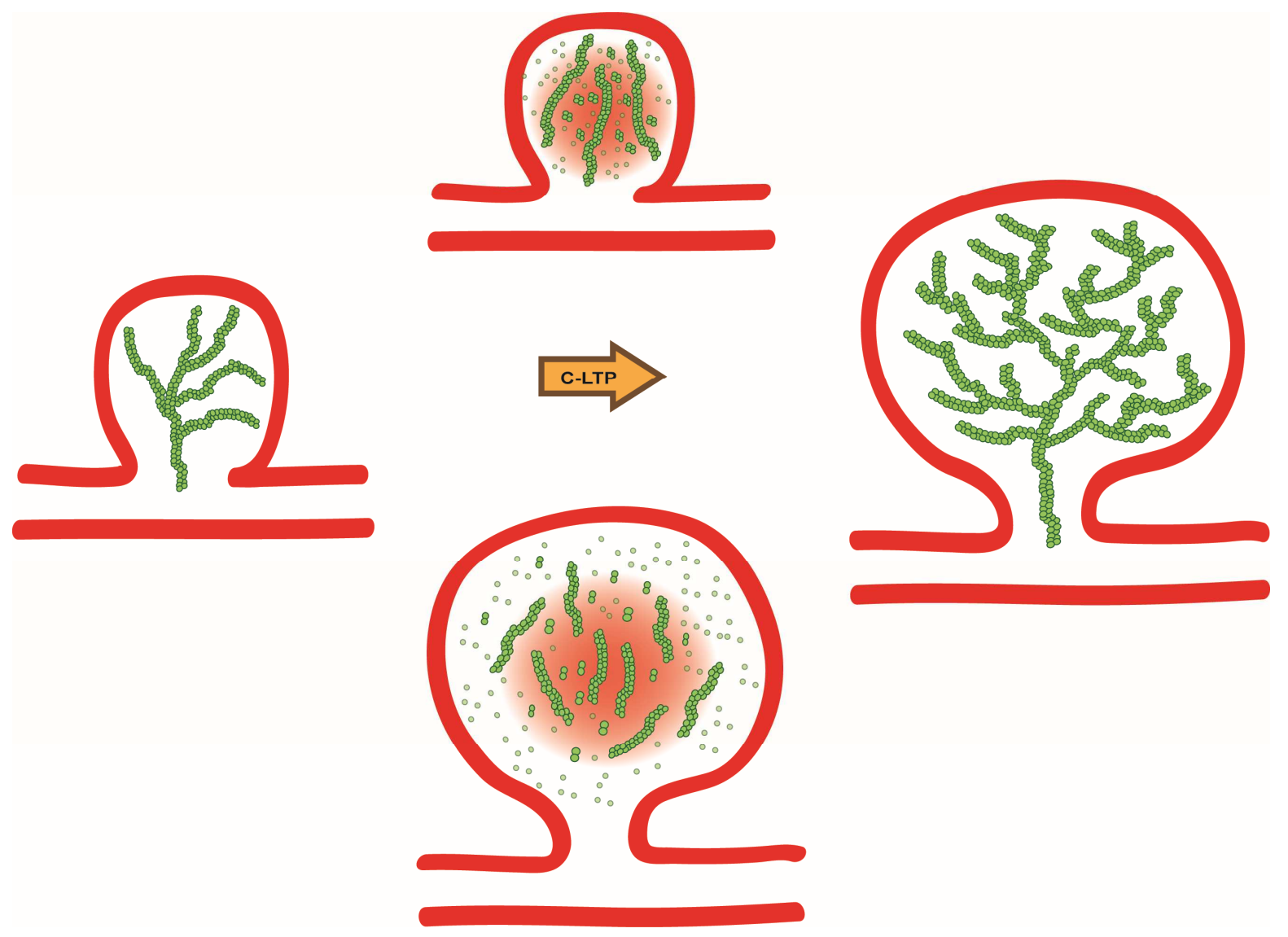

Figure 5.1.1 Reorganization and remodeling of actin filaments within dendritic spine during the process of chemically induced LTP. During the process of LTP, the size of dendritic spine enlarged in response to the long-term potentiation. With the enlargement of dendritic spine, the dynamic actin filaments serve as backbone to facilitate morphological enlargement. 


\section{List of Figures}

Figure 1.1.1. The basic structure of a single neuron cell.................................................... 1

Figure 1.1.2. Overview of the pre- and postsynaptic cells ................................................. 3

Figure 1.1.3. Morphology and size heterogeneity of dendritic spines ................................. 4

Figure 1.1.4. Correlations of synaptic strength with other parameters ................................. 7

Figure 1.1.5. Structures of actin compositions and their functionalities ............................... 8

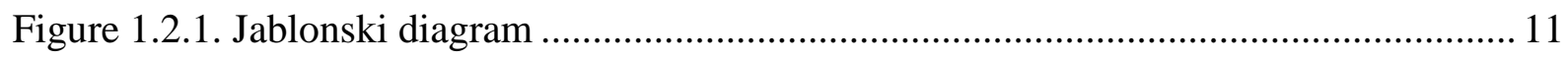

Figure 1.2.2. Illustration of fluorescence quantum yield and lifetime ................................ 15

Figure 1.2.3. Simplified Jablonski diagram to explain two-photon excitation ....................... 15

Figure 1.2.4. Principle of autocorrelation analysis....................................................... 17

Figure 1.2.5. Intensity point spread function (IPSF) of OPE and TPE …............................. 23

Figure 1.2.6. Schematic representation of a wide field fluorescence microscope setup......... 24

Figure 1.2.7. Other methods for molecular diffusion measurements .................................. 26

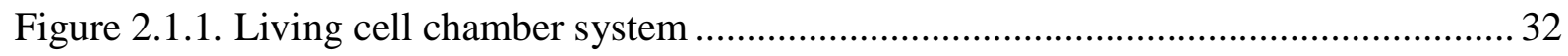

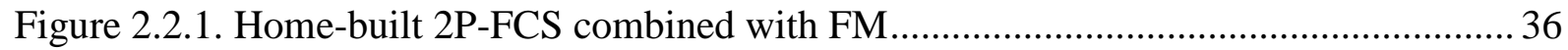

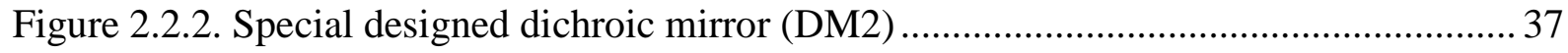

Figure 2.2.3. Co-localization between 1P and 2P excitation in $\mathrm{X}-\mathrm{Y}$ plane............................. 38

Figure 2.2.4. Co-localization between $1 \mathrm{P}$ and $2 \mathrm{P}$ excitation in $\mathrm{Z}$ plane ............................... 39

Figure 3.1.1. Parameters of Annexin V-eGFP obtained from power-dependent FCS

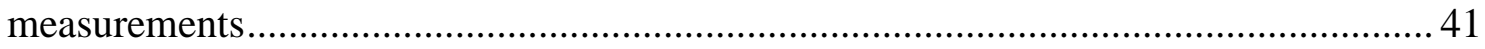

Figure 3.2.1. Homogeneous and heterogeneous diffusion particles ................................... 43

Figure 3.2.2. The fractions between two distinct compositions within the focal volume........ 45

Figure 3.2.3. Statistics and fitting methods to quantify autocorrelation curves of living cells

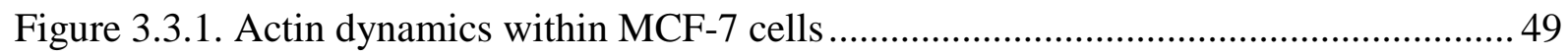

Figure 3.3.2. Actin dynamics within MCF-7 cells treated with Lat-A ................................ 51

Figure 3.3.3. Actin dynamics within dendritic spines treated with Lat-A ............................. 52 
Figure 3.3.4. Changes in actin dynamics and molecular fractions within dendritic spines before and after the treatment of Lat-A

Figure 3.3.5. Changes in particle numbers and brightness within dendritic spines before and after the treatment of Lat-A 55

Figure 3.3.6. Changes in overall intensity before and after Lat-A treatments 56

Figure 3.4.1. Dendritic spines in CA3 hippocampal pyramidal neurons and their morphological changes after cLTP 58

Figure 3.4.2. Statistics of morphological enlargement after cLTP 59

Figure 3.5.1. FRAP imaging of eGFP-actin within dendritic spines 60

Figure 3.5.2. FRAP analysis of eGFP-actin dynamics before and after cLTP 62

Figure 3.5.3. Morphological changes in dendritic spines and the changes of actin dynamics before and after cLTP 63

Figure 3.5.4. Changes in diffusion time and heterogeneity of actin filaments before and after cLTP 65

Figure 3.5.5. Changes of particle number and brightness within dendritic spines before and after cLTP 66

Figure 3.5.6. Changes in overall intensity before and after cLTP.

Figure 3.6.1. Changes in diffusion time and molecular heterogeneity with blockage of cLTP

Figure 3.6.2. Changes in diffusion time and molecular heterogeneity within dendritic spines without morphological changes under TEA treatment 71

Figure 3.6.3. Intrinsic actin dynamics and molecular heterogeneity without any treatment ... 72

Figure 3.6.4. Changes in particle numbers in control experiments 73

Figure 3.6.5. Changes in overall intensity in control experiments. 74

Figure 5.1.1. Reorganization and remodeling of actin filaments within dendritic spine during the process of chemically induced LTP .81 


\section{List of Tables}

Table 1.1 Comparisons of the timescale of various molecular reactions with regard to

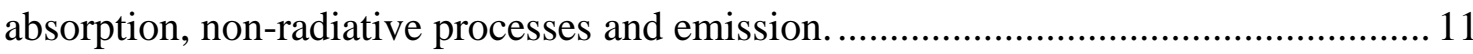

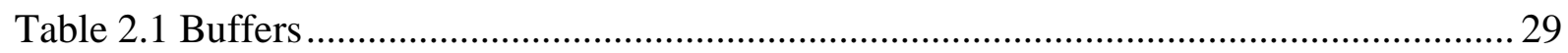

Table 3.1 Summary of physical parameters of actin dynamics before and after Lat-A

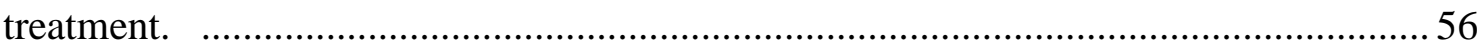

Table 3.2 Comparisons of the changes in different actin pools and turnover time before and

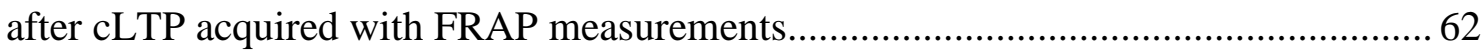

Table 3.3 Summary of physical parameters of actin dynamics before and after TEA treatment 


\section{Abbreviations}

2P-FCS

AMPA

AMPAR

AP5

APD

CCD

cLTP

CMV

CPP

DIV

DM

eGFP

EM

F-actin

FCS

FM

FRAP

FRET

HBSS

IPSF

Lat-A

LTD

LTP
Two photon fluorescence correlation spectroscopy

$\alpha$-amino-3-hydroxy-5-methyl-4-isoxazolepropionic acid

AMPA receptor

2-amino-5-phosphonopentanoic acid

Avalanche photodiode

Charge-couple devices

Chemically induced LTP

Cytomegalovirus

Counts per particle

Days in vitro

Dichroic mirror

Enhanced green fluorescence protein

Electron microscopy

Filamentous actin

Fluorescence correlation spectroscopy

Fluorescence microscopy

Fluorescence recovery after photobleaching

Fluorescence resonance energy transfer

Hank's buffered salt solution

Intensity point spread function

Latrunculin-A

Long-term depression

Long-term potentiation 


$\begin{array}{ll}\text { MCF-7 cell } & \text { Human breast adenocarcinoma cell line } \\ \text { MEM } & \text { Minimum essential medium } \\ \text { MSD } & \text { Mean square displacement } \\ \text { NMDA } & N \text {-methyl-D-aspartate } \\ \text { NMDAR } & \text { NMDA receptor } \\ \text { OPE } & \text { One-photon excitation } \\ \text { PALM } & \text { Photoactivated localization microscopy } \\ \text { PSD } & \text { Postsynaptic density } \\ \text { PSD-95 } & \text { PSD protein of 95 kDa } \\ \text { ROI } & \text { Region of interest } \\ \text { SPT } & \text { Single particle tracking } \\ \text { STED } & \text { Stimulated emission depletion } \\ \text { TEA } & \text { Tetraethylammonium } \\ \text { TPE } & \text { Two-photon excitation }\end{array}$




\section{Publication}

(In Preparation)

Spatial-temporal actin dynamics during synaptic plasticity of single dendritic spine investigated by two photon fluorescence correlation spectroscopy

$\underline{\text { Jian-Hua Chen }}^{1}$, Yves Kellner ${ }^{2}$, Marta Zagrebelsky ${ }^{2}$, Matthias Grunwald ${ }^{1}$, Martin Korte $^{2}$, Peter Jomo Walla ${ }^{1,3}$

${ }^{1}$ AG Biomolecular Spectroscopy and Single-Molecule Detection, Max Planck-Institute for Biophysical Chemistry, Germany

${ }^{2}$ Division of Cellular Neurobiology, Zoological Institute, TU Braunschweig, 38106

Braunschweig, Germany

${ }^{3}$ Department of Biophysical Chemistry, Institute for Physical and Theoretical Chemistry, TU Braunschweig, 38106 Braunschweig, Germany 


\section{Acknowledgment}

First of all, I would like to thank my supervisor, Prof. Dr. Peter Jomo Walla, for giving me this opportunity to work in his group. Also, my sincere thanks to the thesis committee members, Prof. Dr. Reihard Jahn and Prof. Dr. Andreas Janshoff, for their many fruitful discussions regarding my research work.

Thanks to all the group members in Walla's group, specially thanks to Matthias Grunwald, Sabrina Schröder, Dr. Wensi Vennekate and Chao-Chen Lin for their fully help not only in experimental stuff but also in spiritual supporting. I also appreciate Yves Kellner and Dr. Marta Zagrebelsky for their nicely comments about my experimental design and methods. My research work could not have been done without their entirely support. Further, especially many thanks to Inge Dreger, who always reminds me of my lovely mom in Taiwan, for her kindly help upon my arrival in Germany and correcting English for my thesis.

I also would like to thank to all my friends in Göttingen for their unconditional supports and accompany. The most important is that I would like to thank my family, my parents, my sister and my brother, for their love and firmly backup. I cannot have done my study without your regretless accompany and support. 


\title{
10 Curriculum Vitae
}

\section{Personal information}

\author{
Name: Jian-Hua Chen \\ E-mail: jchen1@gwdg.de \\ Gender: Male
}

Date of birth: 27.12 .1979

Place of birth: Nantou, Taiwan

Citizenship: Taiwanese

\section{Education}

2009 - 2013: Ph.D. in the Group of Biomolecular Spectroscopy and SingleMolecule Detection

at the Max Planck Institute for Biophysical Chemistry, Göttingen

within the doctoral program (IMPRS-Physics of biological and complex systems)

of the Georg-August University School of Science (GAUSS)

2002 - 2004: M.Sc. in Science

Institute of Biophotonics

National Yang-Ming University

Taipei, Taiwan

1998 - 2002: B.Sc. in Engineering

Department of Electrical Engineering

National Central University

Zhongli, Taiwan 


\section{Bibliography}

Axelrod D, Koppel DE, Schlessinger J, Elson E, Webb WW (1976) Mobility measurement by analysis of fluorescence photobleaching recovery kinetics. Biophysical journal 16: 1055-1069

Baumgartel V, Muller B, Lamb DC (2012) Quantitative live-cell imaging of human immunodeficiency virus (HIV-1) assembly. Viruses 4: 777-799

Bliss T, Collingridge G (1993) A synaptic model of memory: long-term potentiation in the hippocampus. Nature 361: 31-39

Bliss TV, Lomo T (1973) Long-lasting potentiation of synaptic transmission in the dentate area of the anaesthetized rabbit following stimulation of the perforant path. The Journal of physiology 232: 331-356

Brazda P, Szekeres T, Bravics B, Toth K, Vamosi G, Nagy L (2011) Live-cell fluorescence correlation spectroscopy dissects the role of coregulator exchange and chromatin binding in retinoic acid receptor mobility. Journal of cell science 124: 3631-3642

Chalfie M, Tu Y, Euskirchen G, Ward WW, Prasher DC (1994) Green fluorescent protein as a marker for gene expression. Science (New York, NY) 263: 802-805

Cingolani LA, Goda Y (2008) Actin in action: the interplay between the actin cytoskeleton and synaptic efficacy. Nature reviews Neuroscience 9: 344-356

Collingridge G, Bliss T (1987) NMDA receptors: Their role in long-term potentiation. Trends in Neurosciences 10: 288-293

Coue M, Brenner SL, Spector I, Korn ED (1987) Inhibition of actin polymerization by latrunculin A. FEBS letters 213: 316-318

Culbertson CT, Jacobson SC, Michael Ramsey J (2002) Diffusion coefficient measurements in microfluidic devices. Talanta 56: 365-373

Cypionka A, Stein A, Hernandez JM, Hippchen H, Jahn R, Walla PJ (2009) Discrimination between docking and fusion of liposomes reconstituted with neuronal SNARE-proteins using FCS. PNAS 106: 18575-18580

Dayel MJ, Hom EF, Verkman AS (1999) Diffusion of green fluorescent protein in the aqueous-phase lumen of endoplasmic reticulum. Biophysical journal 76: 2843-2851 
Dertinger T, Pacheco V, von der Hocht I, Hartmann R, Gregor I, Enderlein J (2007) Twofocus fluorescence correlation spectroscopy: a new tool for accurate and absolute diffusion measurements. Chemphyschem 8: 433-443

Dillon C, Goda Y (2005) The actin cytoskeleton: integrating form and function at the synapse. Annual review of neuroscience 28: 25-55

Ding JB, Takasaki KT, Sabatini BL (2009) Supraresolution imaging in brain slices using stimulated-emission depletion two-photon laser scanning microscopy. Neuron 63: 429437

Ehrenberg M, Rigler R (1974) Rotational brownian motion and fluorescence intensify fluctuations. Chemical Physics 4: 390-401

Fischer M, Kaech S, Knutti D (1998) Rapid actin-based plasticity in dendritic spines. Neuron 20: 847-854

Frost NA, Shroff H, Kong H, Betzig E, Blanpied TA (2010) Single-Molecule Discrimination of Discrete Perisynaptic and Distributed Sites of Actin Filament Assembly within Dendritic Spines. Neuron 67: 86-99

Gendron PO, Avaltroni F, Wilkinson KJ (2008) Diffusion coefficients of several rhodamine derivatives as determined by pulsed field gradient-nuclear magnetic resonance and fluorescence correlation spectroscopy. Journal of fluorescence 18: 10931101

Germain RN, Robey EA, Cahalan MD (2012) A decade of imaging cellular motility and interaction dynamics in the immune system. Science (New York, NY) 336: 1676-1681

Gowrishankar K, Ghosh S, Saha S, C R, Mayor S, Rao M (2012) Active remodeling of cortical actin regulates spatiotemporal organization of cell surface molecules. Cell 149: 1353-1367

Gu J, Lee CW, Fan Y, Komlos D, Tang X, Sun C, Yu K, Hartzell HC, Chen G, Bamburg JR, Zheng JQ (2010) ADF/cofilin-mediated actin dynamics regulate AMPA receptor trafficking during synaptic plasticity. Nature neuroscience 13: 1208-1215

Harris KM, Jensen FE, Tsao B (1992) Three-dimensional structure of dendritic spines and synapses in rat hippocampus (CA1) at postnatal day 15 and adult ages: implications for the maturation of synaptic physiology and long-term potentiation. The Journal of neuroscience 12: 2685-2705 
Harvey CD, Yasuda R, Zhong H, Svoboda K (2008) The spread of Ras activity triggered by activation of a single dendritic spine. Science (New York, NY) 321: 136-140

Harvey L, Arnold B, S Lawrence Z, Paul M, David B, James D (2000) Molecular Cell Biology W.H. Freeman and Company.

Heinze KG, Koltermann A, Schwille P (2000) Simultaneous two-photon excitation of distinct labels for dual-color fluorescence crosscorrelation analysis. PNAS 97: 1037710382

Hernandez JM, Stein A, Behrmann E, Riedel D, Cypionka A, Farsi Z, Walla PJ, Raunser S, Jahn R (2012) Membrane fusion intermediates via directional and full assembly of the SNARE complex. Science (New York, NY) 336: 1581-1584

Holtmaat A, Svoboda K (2009) Experience-dependent structural synaptic plasticity in the mammalian brain. Nature reviews Neuroscience 10: 647-658

Honkura N, Matsuzaki M, Noguchi J, Ellis-Davies GCR, Kasai H (2008) The Subspine Organization of Actin Fibers Regulates the Structure and Plasticity of Dendritic Spines. Neuron 57: 719-729

Kasai H, Fukuda M, Watanabe S, Hayashi-Takagi A, Noguchi J (2010) Structural dynamics of dendritic spines in memory and cognition. Trends in Neurosciences 33: 121-129

Kasai H, Matsuzaki M, Noguchi J, Yasumatsu N, Nakahara H (2003) Structure-stabilityfunction relationships of dendritic spines. Trends in neurosciences 26: 360-368

Kennedy MB, Beale HC, Carlisle HJ, Washburn LR (2005) Integration of biochemical signalling in spines. Nature reviews Neuroscience 6: 423-434

Kim E, Sheng M (2004) PDZ domain proteins of synapses. Nature reviews Neuroscience 5: 771-781

Kim SA, Heinze KG, Bacia K, Waxham MN, Schwille P (2005) Two-photon crosscorrelation analysis of intracellular reactions with variable stoichiometry. Biophysical journal 88: 4319-4336

Kim SA, Heinze KG, Schwille P (2007) Fluorescence correlation spectroscopy in living cells. Nature methods 4: 963-973 
Korobova F, Svitkina T (2010) Molecular architecture of synaptic actin cytoskeleton in hippocampal neurons reveals a mechanism of dendritic spine morphogenesis. Molecular biology of the cell 21: 165-176

Krucker T, Siggins GR, Halpain S (2000) Dynamic actin filaments are required for stable long-term potentiation (LTP) in area CA1 of the hippocampus. Proceedings of the National Academy of Sciences of the United States of America 97: 6856-6861

Kusumi A, Sako Y, Yamamoto M (1993) Confined lateral diffusion of membrane receptors as studied by single particle tracking (nanovid microscopy). Effects of calciuminduced differentiation in cultured epithelial cells. Biophysical journal 65: 2021-2040

Lakowicz JR (2006) Principles of fluorescence spectroscopy, Vol. 1: Springer.

Lamprecht R, LeDoux J (2004) Structural plasticity and memory. Nature reviews Neuroscience 5: 45-54

Lee SJ, Escobedo-Lozoya Y, Szatmari EM, Yasuda R (2009) Activation of CaMKII in single dendritic spines during long-term potentiation. Nature 458: 299-304

Levitan IB, Kaczmarek LK (2002) The Neuron, Cell and molecular Biology: Oxford University Express.

Lin Y, Koleske AJ (2010) Mechanisms of Synapse and Dendrite Maintenance and Their Disruption in Psychiatric and Neurodegenerative Disorders. Annual review of neuroscience 33: 349-378

Lisman J, Yasuda R, Raghavachari S (2012) Mechanisms of CaMKII action in long-term potentiation. Nature reviews Neuroscience 13: 169-182

Magde D, Elson E, Webb WW (1972) Thermodynamic Fluctuations in a Reacting System-Measurement by Fluorescence Correlation Spectroscopy. Physical Review Letters 29: 705-708

Magde D, Elson EL, Webb WW (1974) Fluorescence correlation spectroscopy. II. An experimental realization. Biopolymers 13: 29-61

Maletic-Savatic M, Malinow R, Svoboda K (1999) Rapid dendritic morphogenesis in CA1 hippocampal dendrites induced by synaptic activity. Science (New York, NY) 283: 19231927 
Malinow R, Malenka RC (2002) AMPA receptor trafficking and synaptic plasticity. Annual review of neuroscience 25: 103-126

Matsuzaki M, Honkura N, Ellis-Davies GC, Kasai H (2004) Structural basis of long-term potentiation in single dendritic spines. Nature 429: 761-766

Morris RG, Anderson E, Lynch GS, Baudry M (1986) Selective impairment of learning and blockade of long-term potentiation by an N-methyl-D-aspartate receptor antagonist, AP5. Nature 319: 774-776

Mueller V, Ringemann C, Honigmann A, Schwarzmann G, Medda R, Leutenegger M, Polyakova S, Belov VN, Hell SW, Eggeling C (2011) STED nanoscopy reveals molecular details of cholesterol- and cytoskeleton-modulated lipid interactions in living cells. Biophysical journal 101: 1651-1660

Müller CB, Loman A, Pacheco V, Koberling F, Willbold D, Richtering W, Enderlein J (2008) Precise measurement of diffusion by multi-color dual-focus fluorescence correlation spectroscopy. EPL (Europhysics Letters) 83: 46001

Murakoshi H, Wang H, Yasuda R (2011) Local, persistent activation of Rho GTPases during plasticity of single dendritic spines. Nature 472: 100-104

Nagerl UV, Willig KI, Hein B, Hell SW, Bonhoeffer T (2008) Live-cell imaging of dendritic spines by STED microscopy. PNAS 105: 18982-18987

Nath S, Meuvis J, Hendrix J, Carl SA, Engelborghs Y (2010) Early aggregation steps in alpha-synuclein as measured by FCS and FRET: evidence for a contagious conformational change. Biophysical journal 98: 1302-1311

Okamoto K, Nagai T, Miyawaki A (2004) Rapid and persistent modulation of actin dynamics regulates postsynaptic reorganization underlying bidirectional plasticity. Nature neuroscience 7: 1104-1112

Partikian A, Olveczky B, Swaminathan R, Li Y, Verkman AS (1998) Rapid diffusion of green fluorescent protein in the mitochondrial matrix. The Journal of cell biology 140: 821-829

Petrasek Z, Schwille P (2008) Photobleaching in two-photon scanning fluorescence correlation spectroscopy. Chemphyschem 9: 147-158

Pollard TD, Borisy GG (2003) Cellular motility driven by assembly and disassembly of actin filaments. Cell 112: 453-465 
Pollard TD, Cooper JA (2009) Actin, a central player in cell shape and movement. Science (New York, NY) 326: 1208-1212

Price ES, Aleksiejew M, Johnson CK (2011) FRET-FCS detection of intralobe dynamics in calmodulin. The journal of physical chemistry $B$ 115: 9320-9326

Qian H, Sheetz MP, Elson EL (1991) Single particle tracking. Analysis of diffusion and flow in two-dimensional systems. Biophysical journal 60: 910-921

Remaut K, Lucas B, Braeckmans K, Sanders NN, De Smedt SC, Demeester J (2005) FRETFCS as a tool to evaluate the stability of oligonucleotide drugs after intracellular delivery. Journal of controlled release 103: 259-271

Rhoades E, Ramlall TF, Webb WW, Eliezer D (2006) Quantification of alpha-synuclein binding to lipid vesicles using fluorescence correlation spectroscopy. Biophysical journal 90: $4692-4700$

Rigler R, Mets Ü, Widengren J, Kask P (1993) Fluorescence correlation spectroscopy with high count rate and low background: analysis of translational diffusion. European Biophysics Journal 22: 169-175

Roth-Alpermann C, Morris RG, Korte M, Bonhoeffer T (2006) Homeostatic shutdown of long-term potentiation in the adult hippocampus. PNAS 103

Rouse JPE (1953) A Theory of the Linear Viscoelastic Properties of Dilute Solutions of Coiling Polymers. The Journal of Chemical Physics 21: 1272-1280

Ruddies R, Goldmann WH, Isenberg G, Sackmann E (1993) The viscoelasticity of entangled actin networks: the influence of defects and modulation by talin and vinculin. European Biophysics Journal 22: 309-321

Rusu L, Gambhir A, McLaughlin S, Radler J (2004) Fluorescence correlation spectroscopy studies of Peptide and protein binding to phospholipid vesicles. Biophysical journal 87: 1044-1053

Schlessinger J, Koppel DE, Axelrod D, Jacobson K, Webb WW, Elson EL (1976) Lateral transport on cell membranes: mobility of concanavalin A receptors on myoblasts. PNAS 73: $2409-2413$

Schmid SM, Hollmann M (2010) Bridging the synaptic cleft: lessons from orphan glutamate receptors. Science signaling 3: pe28 
Schwille P, Haupts U, Maiti S, Webb WW (1999) Molecular dynamics in living cells observed by fluorescence correlation spectroscopy with one- and two-photon excitation. Biophysical journal 77: 2251-2265

Sezgin E, Levental I, Grzybek M, Schwarzmann G, Mueller V, Honigmann A, Belov VN, Eggeling C, Coskun U, Simons K, Schwille P (2012) Partitioning, diffusion, and ligand binding of raft lipid analogs in model and cellular plasma membranes. Biochimica et biophysica acta 1818: 1777-1784

Shaner NC, Campbell RE, Steinbach PA, Giepmans BN, Palmer AE, Tsien RY (2004) Improved monomeric red, orange and yellow fluorescent proteins derived from Discosoma sp. red fluorescent protein. Nature biotechnology 22: 1567-1572

Sheng M, Hoogenraad CC (2007) The postsynaptic architecture of excitatory synapses: a more quantitative view. Annual review of biochemistry 76: 823-847

Spruston N (2008) Pyramidal neurons: dendritic structure and synaptic integration. Nature reviews Neuroscience 9: 206-221

Star E, Kwiatkowski D, Murthy V (2002) Rapid turnover of actin in dendritic spines and its regulation by activity. Nature neuroscience 5: 239-246

Stasevich TJ, Mueller F, Michelman-Ribeiro A, Rosales T, Knutson JR, McNally JG (2010) Cross-validating FRAP and FCS to quantify the impact of photobleaching on in vivo binding estimates. Biophysical journal 99: 3093-3101

Stoppini L, Buchs PA, Muller D (1991) A simple method for organotypic cultures of nervous tissue. Journal of neuroscience methods 37: 173-182

Svoboda K, Tank DW, Denk W (1996) Direct measurement of coupling between dendritic spines and shafts. Science (New York, NY) 272: 716-719

Tada T, Sheng M (2006) Molecular mechanisms of dendritic spine morphogenesis. Current opinion in neurobiology 16: 95-101

Tatavarty V, Kim EJ, Rodionov V, Yu J (2009) Investigating sub-spine actin dynamics in rat hippocampal neurons with super-resolution optical imaging. PloS one 4: e7724

Toomre D (2012) Cellular imaging using total internal reflection fluorescence microscopy: theory and instrumentation. Cold Spring Harbor protocols 2012: 414-424 
Tsay JM, Doose S, Weiss S (2006) Rotational and translational diffusion of peptidecoated CdSe/CdS/ZnS nanorods studied by fluorescence correlation spectroscopy. Journal of the American Chemical Society 128: 1639-1647

Tsien RY (1998) The green fluorescent protein. Annual review of biochemistry 67: 509544

Urban NT, Willig KI, Hell SW, Nagerl UV (2011) STED nanoscopy of actin dynamics in synapses deep inside living brain slices. Biophysical journal 101: 1277-1284

Vennekate W, Schroder S, Lin CC, van den Bogaart G, Grunwald M, Jahn R, Walla PJ (2012) Cis- and trans-membrane interactions of synaptotagmin-1. PNAS 109: 11037-11042

Walla PJ (2009) Modern Biophysical Chemistry-Detection and Analysis of Biomolecules: Wiley-VCH, Weinheim.

Weiss M, Hashimoto H, Nilsson T (2003) Anomalous protein diffusion in living cells as seen by fluorescence correlation spectroscopy. Biophysical journal 84: 4043-4052

Yarmola EG, Somasundaram T, Boring TA, Spector I, Bubb MR (2000) Actin-latrunculin A structure and function. Differential modulation of actin-binding protein function by latrunculin A. The Journal of biological chemistry 275: 28120-28127

Yasuda R, Harvey CD, Zhong H, Sobczyk A, van Aelst L, Svoboda K (2006) Supersensitive Ras activation in dendrites and spines revealed by two-photon fluorescence lifetime imaging. Nature neuroscience 9: 283-291

Yuste R, Bonhoeffer T (2001) Morphological changes in dendritic spines associated with long-term synaptic plasticity. Annual review of neuroscience 24: 1071-1089

Zhou Q, Homma KJ, Poo MM (2004) Shrinkage of dendritic spines associated with longterm depression of hippocampal synapses. Neuron 44: 749-757

Zipfel WR, Williams RM, Webb WW (2003) Nonlinear magic: multiphoton microscopy in the biosciences. Nature biotechnology 21: 1369-1377

Zwilling D, Cypionka A, Pohl WH, Fasshauer D, Walla PJ, Wahl MC, Jahn R (2007) Early endosomal SNAREs form a structurally conserved SNARE complex and fuse liposomes with multiple topologies. The EMBO journal 26: 9-18 